\title{
Resonant Inelastic X-ray Scattering (RIXS) Studies in Chemistry: Present and Future
}

Marcus Lundberg, Philippe Wernet

\section{Contents}

Introduction

Principles of RIXS for $3 d$ transition-metal complexes

Soft X-ray RIXS: Metal L-edges

Hard X-ray RIXS: Metal K-edges

Applications of Metal L-edge RIXS

Ligand-field excitations in metal complexes and nanoparticles

Metal-ligand covalency from charge-transfer excitations

Applications of Metal K-edge RIXS

Electron Transfer in Cytochrome C

Cubane-like Co Cluster of Relevance for Water Oxidation

Time-resolved RIXS for characterizing transient reaction intermediates

Conclusions and Outlook

References 
Marcus Lundberg (marcus.lundberg@kemi.uu.se)

Department of Chemistry - Ångström Laboratory, Uppsala University, SE-75121 Uppsala, Sweden

Added after acceptance: New e-mail address: philippe.wernet@physics.uu.se

Philippe Wernet (wernet@helmholtz-berlin.de)

Institute for Methods and Instrumentation for Synchrotron Radiation Research, HelmholtzZentrum Berlin für Materialien und Energie GmbH, 12489 Berlin, Germany 


\begin{abstract}
This chapter illustrates how resonant inelastic x-ray scattering (RIXS) is used to address questions in chemistry, with special focus on the electronic structure and catalytic activity of first row transition metals. RIXS is a two-photon process that is the $\mathrm{x}$-ray equivalent of resonance Raman spectroscopy. The final states correspond to vibrational, valence electronic or even core excitations. In addition to the advantages of a local element-selective $\mathrm{x}$-ray spectroscopic probe, RIXS gives new information compared to single-photon $\mathrm{x}$-ray absorption and x-ray emission experiments. Metal L-edge RIXS shows intense metalcentered ligand-field transitions, even in cases where they are spin or parity forbidden in optical absorption spectroscopy. By selecting different resonances by appropriately tuning the incident energy, it is possible to isolate different ligand-field and charge-transfer transitions. The observation of a large number of electronic states that can be properly assigned, sometimes with the help of theoretical methods, gives novel opportunities to quantify metal-ligand interactions and their contributions to reactivity. RIXS in the $\mathrm{K}$ preedge can be used to obtain L- and M-edge like spectra including insight into charge-transfer excitations all with the advantages of a hard x-ray probe. Finally, it is shown how timeresolved RIXS down to the femtosecond timescale probes the orbitals of transient reaction intermediates. The usefulness of RIXS in chemistry is shown for a diverse set of systems, including coordination complexes, metal enzymes and nanoparticles.
\end{abstract}

\title{
Keywords
}

X-ray free-electron laser, resonant inelastic x-ray scattering, RIXS, coordination complex, metalloprotein, nanoparticle, ligand-field excitation, covalency, transient intermediate, transition metals, electronic structure calculations

\section{Glossary}

RIXS Resonant Inelastic X-ray Scattering 


$\begin{array}{ll}\text { XES } & \text { X-ray Emission Spectroscopy } \\ \text { EXAFS } & \text { Extended X-ray Absorption Fin Structure Spectroscopy } \\ \text { XPS } & \text { X-ray Photoelectron Spectroscopy } \\ \text { SR } & \text { Synchrotron Radiation } \\ \text { XFEL } & \text { X-ray Free-Electron Laser } \\ \text { LF } & \text { Ligand field } \\ \text { CT } & \text { Charge transfer } \\ \text { LMCT } & \text { Ligand to Metal Charge Transfer } \\ \text { MLCT } & \text { Metal to Ligand Charge Transfer } \\ \text { CFM } & \text { Crystal-Field Multiplet } \\ \text { CTM } & \text { Charge-Transfer Multiplet } \\ \text { RAS } & \text { Restricted Active Space }\end{array}$




\section{Introduction}

Resonant inelastic x-ray scattering (RIXS) is the x-ray analog of resonance Raman scattering. Figure 1a depicts this analogy where it is shown that with optical light in conventional resonance Raman scattering one probes vibrationally excited states of the system, while with $\mathrm{x}$-ray radiation in RIXS electronic excited states are probed. The chapters by Braicovich, Nordgren and Rubensson, and by Brookes in this book also treat RIXS and the reader is encouraged to consider their presentations of the basic principles of this method as well. Nordgren and Rubensson also discuss the exceptional case where RIXS is used to probe vibrational excitations. Here we focus on applications of RIXS to problems in chemistry. We also refer to the aforementioned chapters in this book for more detailed technical considerations of how to implement RIXS just mentioning one important aspect here for applications in chemistry. Due to the necessity to tune the incident $\mathrm{x}$-ray energy with a comparably small bandwidth and in order to probe the often dilute chemical species, RIXS is performed at highly brilliant synchrotron radiation (SR) or x-ray free-electron laser (XFEL) sources.

For applications of RIXS in chemistry it is important to realize that RIXS gives access to the valence electrons thereby giving access to the frontier orbitals that determine chemical bonding. Depending on the implementation and depending on, in particular, whether soft or hard x-rays are used to perform the RIXS experiment, the final electronic excited states can be valence- or core-excited states. We discuss here how these different states represent different ways to access the valence electrons in chemically relevant systems. When valenceexcited states are probed with RIXS, the essential transitions between the frontier orbitals that determine the chemical bonding are accessed. This is where an additional analogy between RIXS and another commonly used method for the investigation of chemical systems becomes apparent: The final valence-excited states of RIXS are, to first approximation, the same as for UV/Vis spectroscopy and we show that the selection rules of the two methods can be different giving access to different properties.

Given that various optical methods exist to study the electronic structure in chemical systems, the question is why should one use RIXS to solve problems in chemistry? For many applications in chemistry and in particular for the classes of systems that this chapter focuses on, RIXS offers a unique capability compared to UV/Vis spectroscopy and resonance 
Raman scattering or to optical techniques in general: The intermediate state in RIXS (Figure 1a) is a core-excited state reached by promoting an electron from a core orbital to an unoccupied molecular orbital and this makes RIXS, as any other core-level x-ray spectroscopic method, element- and chemical site specific. Because core-orbitals are spatially well localized RIXS is element-specific and due to the chemical shift of core-level energies RIXS is chemical site-specific. At the selected element and chemical site, the x-ray resonance can even be specific to the promotion of the core electron to a specific molecular orbital, thereby making it orbital specific. In this way RIXS offers the opportunity to extract essential chemical properties locally at the probed site and, possibly, for selected orbitals.
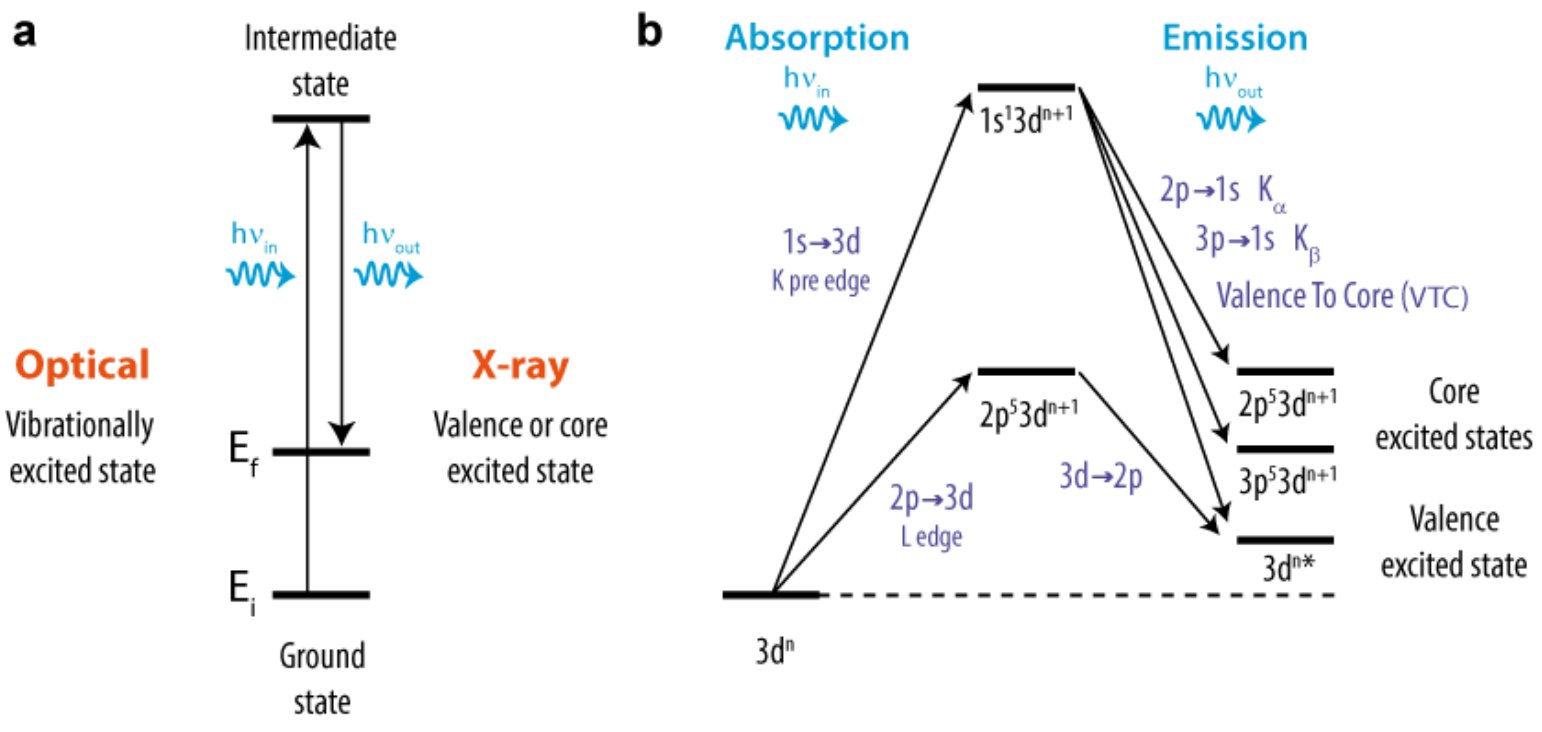

Figure 1. Representations of the resonant inelastic x-ray scattering (RIXS) process. a RIXS as the x-ray analog of resonance Raman scattering. Stokes scattering using optical/x-ray wavelengths are used to probe vibrational/electronic excitations. b Schematic of the RIXS processes for $3 \mathrm{~d}$ transition-metal systems at hard (K-edge) and soft (L-edge) x-ray absorption edges.

This selectivity cannot be overrated and unique insight into a number of important classes of chemical systems has been gained over the past years. Because this chapter is not supposed to be a complete review of all these applications we limit ourselves to few illustrative examples. In addition we focus here on the principles and applications of RIXS for the investigation of first-row transition metals in transition-metal complexes and compounds in inorganic and bio-inorganic systems such as metalloproteins as well as in catalytic systems and in photochemical reactions. This completely subjective choice is motivated by our conviction that when addressing these systems one profits most from the advantages that RIXS offers compared to more conventional non-x-ray techniques. 
Still, to indicate the breadth of the applications of RIXS in chemistry we briefly mention some of the most recent areas with selected examples, again recognizing that it is impossible here to review all studies. In organic chemistry, RIXS at the $\mathrm{N}$ and $\mathrm{O}$ K-edges in the soft $\mathrm{x}$-ray range can be used to study the hydrogen-bonding interactions of $\mathrm{N}-\mathrm{H}$ or $\mathrm{O}-\mathrm{H}$ groups for solvated molecules or in solvents (Weinhardt et al., 2015, Harada et al., 2006). A special case here is the application of RIXS to the study of water and aqueous solutions and the chapter by Nilsson in this book includes this. Other noteworthy applications that reach over to molecular physics, address small molecules in the gas phase focusing on the dynamics in the core-excited intermediate states of RIXS (Marchenko et al., 2015, Zitnik et al., 2015). It has also been demonstrated that with high-enough spectral resolution, vibrational excitations can be probed with RIXS, (Vaz da Cruz et al., 2017), (Chapter by Nordgren Rubensson in this book). Finally, the high selectivity of RIXS to certain elements and sites was demonstrated in its application to bonding and ultrafast dynamics of molecules on surfaces (Dell'Angela et al., 2013, Nilsson and Pettersson, 2004, Öström et al., 2015).

Here we concentrate on the aspects necessary to understand RIXS of metal complexes and related systems and we start by motivating the use of RIXS for these systems. First-row transition metals are important components of many catalytic systems. The development of more efficient and selective catalysts requires knowledge of the electronic structure of the metal $3 d$ orbitals that act as electron donors or acceptors in chemical reactions. One of the most direct ways of accessing these essential $3 d$ frontier orbitals is to investigate the ligandfield spectra of the transition metals (Figgis, 1966). Optical techniques such as UV/Vis spectroscopy are commonly used to measure ligand-field spectra of metal atoms and ions in a variety of materials. However, the important ligand-field (LF) excitations in the range from 0 to 3-5 eV are difficult to access with conventional optical techniques (LF excitations are often also denoted metal-centered, MC, excitations or d-d excitations). In addition they are often weak because they are mostly electric dipole forbidden. Furthermore, they are hard to measure when lying below $0.5-1 \mathrm{eV}\left(4000-8000 \mathrm{~cm}^{-1}\right)$ or above $5 \mathrm{eV}(250 \mathrm{~nm})$ because of instrumental limitations in these energy ranges. Finally, they may be obscured by the chargetransfer (CT) excitations that often dominate UV/Vis spectra. In general, if more than one absorber is present, the optical absorption signals from the metal site cannot be distinguished from absorption by the surrounding ligands and solvent. The element- and site-selectivity of $x$-ray spectroscopy justifies why $x$-ray absorption spectroscopy (XAS), $x$-ray 
emission spectroscopy (XES) and x-ray photoelectron spectroscopy (XPS) are widely used methods to study metal sites (Baker et al., 2017, Koziej and DeBeer, 2017, MacMillan and Lancaster, 2017, De Groot and Kotani, 2008). RIXS can now be regarded as an extension of these methods and it is the purpose of this chapter to demonstrate how RIXS complements these more commonly used $\mathrm{x}$-ray spectroscopy methods.

This chapter is outlined as follows: We start with basic principles of RIXS for studying valence orbitals of metal complexes using the well-known iron hexacyanides addressing both soft $\mathrm{x}$ ray RIXS at the metal L-edges and hard X-ray RIXS at the metal K-edges. With this we show that RIXS does not only overcome some of the limitations of UV/Vis, XAS or XES, but also provides additional electronic-structure information. This is followed by illustrative examples of how RIXS can give chemical insight into the ligand-field excitations and metal-ligand covalency of metal complexes, nanoparticles, synthetic catalysts and metal enzymes. The chapter ends with a brief description of recent time-resolved RIXS experiments on transitionmetal complexes in solution.

\section{Principles of RIXS for 3d transition-metal complexes}

The x-ray resonance Raman process or RIXS can be viewed as a two-photon process, where the energy of the incident photon $(\Omega)$ is tuned into an x-ray absorption resonance, followed by emission of a scattered photon ( $\omega$ ) (Gel'mukhanov and Ågren, 1999, Kotani and Shin, 2001, Ament et al., 2011). If the two photons have the same energy, the scattering is elastic (Rayleigh scattering) and the system returns to the ground state. If the photon loses energy, this inelastic scattering is a Stokes process, see Figure 1a. The energy difference between incident and emitted photons, i.e. the energy transfer $(\Omega-\omega)$, corresponds to the energy of the excitation in the sample (Kao et al., 1996, Butorin et al., 1996c, Butorin et al., 1996b, Platzman and Isaacs, 1998). For transition-metal complexes this is a valence excitation, or, depending on the incident photon energy range, a core excitation from a higher-lying shell. We do not treat here high-resolution experiments that access vibrational transitions (see Introduction) because to the best of our knowledge such experiments have not yet been realized for metal complexes at the time of publication of this chapter. To complete the comparison with resonance Raman, anti-Stokes processes are also possible. For this to be observed in RIXS, the system has to be in a valence excited state and as valence excited 
states cannot be reached by thermal excitation, anti-Stokes RIXS can only be observed if the sample is first electronically excited in a pump-probe experiment. This will be addressed later in this chapter.

Any RIXS measurement has two basic variables, the choice of incident photon energies from the source and the range of emission energies to collect. The application of this concept to RIXS of metal complexes and related systems is discussed with Figure 1b. Starting with the incident energy, metal L-edge XAS ( $2 p \rightarrow 3 d$ excitations) has been extensively used to study metal $3 d$ orbitals. In metal L-edge RIXS the incident energy matches the $2 p$ excitations, and the $2 p$ hole is then filled by an electron from an occupied valence orbital. Incident and emission energy ranges are similar (they only differ by the energy of valence excitations) and the final states in L-edge RIXS are ground or valence excited states (Figure 1b). RIXS final states involving the metal-dominated set of orbitals constitute LF excitations with effective one-electron transitions within the set of metal-dominated orbitals. Final states involving the ligand-dominated set of orbitals correspond to CT excitations with effective one-electron transitions from the ligand-dominated to the metal-dominated set of orbitals.

Spectroscopy in the soft X-ray region presents specific challenges for biological and solution samples (Kubin et al., 2017), for which the standard probe has been the metal K-edge, which lies in the hard $\mathrm{x}$-ray region. The most direct K-edge probes of the reactive $3 \mathrm{~d}$ orbitals are the metal K pre-edges, (Baker et al., 2017, Bergmann and Glatzel, 2009), that are typically assigned to $1 \mathrm{~s} \rightarrow 3 \mathrm{~d}$ excitations (Figure $1 \mathrm{~b}$ ). For core-excited states reached by K-edge absorption with a hole in the deep-lying 1 s orbital, there are many possible decay channels via fluorescence emission as illustrated in Figure $1 \mathrm{~b}$ and hence many choices for which emission to collect. These include $K \alpha$ emission $(2 p \rightarrow 1 s), K \beta$ emission $(3 p \rightarrow 1 s)$ and even valence excited states which can be reached with valence-to-core (VTC) emission (Figure 1b), (Glatzel and Bergmann, 2005, Pollock and DeBeer, 2015). K $\alpha$ and K $\beta$ XES is performed at constant incident photon energy (often chosen to lie well above the $\mathrm{K}$ absorption edge). For RIXS in contrast, the incident energy is tuned through the $K$ absorption pre-edge $(1 s \rightarrow 3 d$ excitation) but the same emission channels are detected resulting in $1 \mathrm{~s} 2 \mathrm{p}$ RIXS (for K $\alpha$ $2 p \rightarrow 1 s$ emission) and 1s3p RIXS (for $K \beta 3 p \rightarrow 1 s$ emission). The final states in $1 s 2 p$ RIXS therefore are states with a $2 p$ hole ( $2 p$ core-excited states), similar to L-edge XAS, while in $1 s 3 p$ RIXS they are states with a $3 p$ hole ( $3 p$ core-excited states). 
The basic principles of $L$ and K-edge RIXS are illustrated with data from the iron hexacyanides in ferric and ferrous oxidation states $\left[\mathrm{Fe}(\mathrm{CN})_{6}\right]^{3-}$ and $\left[\mathrm{Fe}(\mathrm{CN})_{6}\right]^{4-}$. They have been studied extensively with RIXS, e.g. L-edge RIXS for ground as well as short-lived valence excited states in solution (Engel et al., 2014, Kunnus et al., 2016c, Jay et al., 2018a), and K-edge RIXS for the ground states in both solids and in solution (Lundberg et al., 2013, Penfold et al., 2014).

The ferricyanide anion has a formal Fe $\mathrm{e}^{\text {III }}$ oxidation state with a $3 \mathrm{~d}^{5}$ configuration whereas ferrocyanide is $\mathrm{Fe}^{\prime \prime}$ with a $3 \mathrm{~d}^{6}$ configuration. Chemical bonding in these complexes is introduced with Figure 2. Both complexes can be described as having an octahedral $\left(\mathrm{O}_{\mathrm{h}}\right)$ ligand environment with a small Jahn-Teller distortion in ferricyanide. Metal-ligand bonding occurs mainly through the interaction of the iron $3 \mathrm{~d}$ orbitals with occupied $\mathrm{CN}^{-}$valence orbitals (electron donation) as well as with unoccupied orbitals (backdonation). The most important filled ligand orbitals are $5 \sigma$ and $1 \pi$, see Figure 2. Two ligand $\sigma$ orbitals of $e_{g}$ symmetry mix with the metal $3 d$ orbitals to give ligand-dominated bonding $(\sigma)$ and metaldominated antibonding orbitals ( $\sigma^{*}$ typically referred to as $e_{g}$ ) that describe $\sigma$ donation. The most important unoccupied ligand orbitals are $2 \pi$, see Figure 2 . Mixing between metal and three empty $\pi$-type orbitals of $t_{2 g}$ symmetry gives metal-centered weakly bonding ( $\pi$ typically referred to as $\left.t_{2 g}\right)$ and ligand-centered antibonding $\left(\pi^{*}\right)$ orbitals that describe $\pi$ backdonation. We restrict ourselves here to the inclusion of the essential ligand to metal $\sigma$ donation and metal to ligand $\pi$ backdonation interactions but note that for a complete and more accurate description of bonding in metal complexes all bonding, non-bonding and antibonding interactions such as, in addition, ligand to metal $\pi$ donation and metal to ligand $\sigma$ back donation need to be included. 


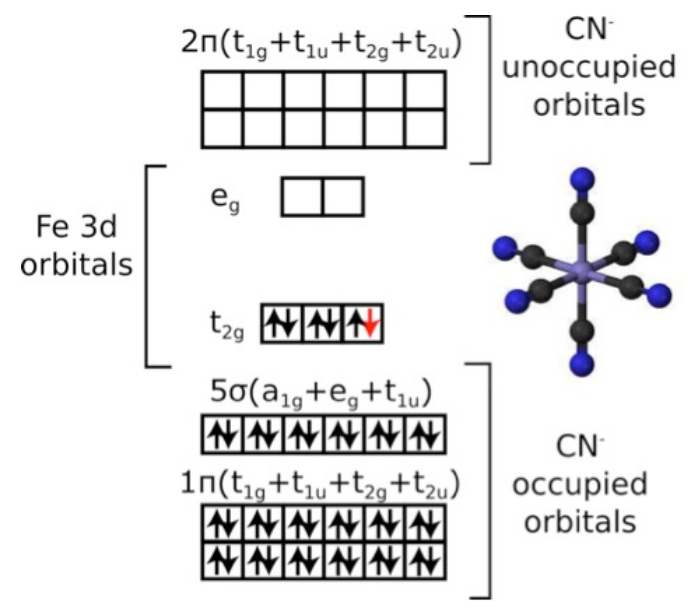

Figure 2. Orbital-energy diagram of iron hexacyanide complexes including the five iron $3 \mathrm{~d}$ orbitals, as well as the $\mathrm{CN}^{-} 5 \sigma$ (one per ligand), $1 \pi$ (two per ligand), $2 \pi$ (two per ligand) orbitals. Without/with the additional red-marked electron this corresponds to ferric/ferrous cyanide. Reprinted with permission from (Kunnus et al., 2016c). Copyright 2016 American Chemical Society.

The iron hexacyanides include all types of bonding contributions, which make them ideal to test an experimental probe of electronic structure and metal-ligand bonding. The combination of strong $\sigma$ donation, which increases the energies of $e_{g}$ orbitals, with $\pi$ back donation, which lowers the energy of the $t_{2 g}$ orbitals, gives a large ligand-field splitting. The $\mathrm{x}$-ray transitions can then be relatively well described by a molecular orbital picture because there is almost no mixing between configurations with different orbital occupations (Butorin, 2000).

\section{Soft X-ray RIXS: Metal L edges}

The metal L-edge RIXS process can in its most basic form be described as a $2 p \rightarrow 3 d$ excitation followed by an electron from a $3 d$-type orbital filling the $2 p$ hole (Figure $1 b$ ). The process can therefore, to first approximation, be seen as a direct way to probe valence excitations using an element-specific probe. As will be shown below, L-edge RIXS has even more advantages than that. These include the enhancement of ligand-field transitions, access to states of different multiplicity, and clear separation between different types of excitations in the twodimensional RIXS map. These intrinsic advantages have made L-edge RIXS a commonly used tool for a wide range of $3 \mathrm{~d}$ transition-metal based materials and they are discussed in the examples treated in this chapter. 
For the particular case of transition metals in solution, experiments are still comparably scarce because only relatively recently have in-vacuum methods for soft $x$-ray RIXS spectroscopy on liquids and solutions been developed (Guo et al., 2002, Lange et al., 2010, Kunnus et al., 2012). Typical count rates are still low because of the small fluorescence yields at metal L-edges and the strong absorption in the soft $\mathrm{x}$-ray range by the light elements $\mathrm{C}, \mathrm{O}$ and $\mathrm{N}$ in typical solvents. The high solubility of the iron hexacyanides in water therefore makes them ideal systems for testing the experimental capabilities of solution-phase RIXS (Engel et al., 2014, Kunnus et al., 2016c, Jay et al., 2018a).

The Fe 2p3d RIXS maps of the two iron hexacyanide complexes as measured in solution are shown in Figure 3 together with their corresponding L-edge XAS spectra (Kunnus et al., 2016c). Starting with the absorption spectra in the top panels in Figure 3, the main features are typically assigned using a molecular orbital picture. In ferricyanide the ground state configuration is $t_{2 g}{ }^{5} e_{g}{ }^{0}$ and the first XAS peak in the $L_{3}$-edge close to $706 \mathrm{eV}$ is a $2 p \rightarrow t_{2 g}$ transition to the lowest-lying $t_{2 g}$ orbitals, see Figure 3a. The next XAS resonance at $710 \mathrm{eV}$ corresponds to excitations to the next higher-lying $e_{g}$ orbitals with $2 p \rightarrow e_{g}$ transitions and the third resonance peak at $712 \mathrm{eV}$ is typically labeled $\pi^{*}$ and due to $2 p \rightarrow \pi^{*}$ transitions (here * denotes antibonding orbitals). This molecular orbital picture is not strictly accurate because the final states of the third XAS resonance peak are strongly mixed $e_{g} / \pi^{*}$ configurations rather than direct excitations to the molecular $\pi^{*}$ orbital. Still, the peak is a strong signature of $\pi$ backdonation thereby justifying the notation. Ferrocyanide has $\mathrm{t}_{2 \mathrm{~g}}{ }^{6} \mathrm{e}_{\mathrm{g}}{ }^{0}$ ground state and therefore lacks the $t_{2 g}$ XAS resonance peak but shows both $e_{g}$ and $\pi^{*}$ peaks, at 709 and $711 \mathrm{eV}$, respectively, see Figure $3 \mathrm{~b}$. 

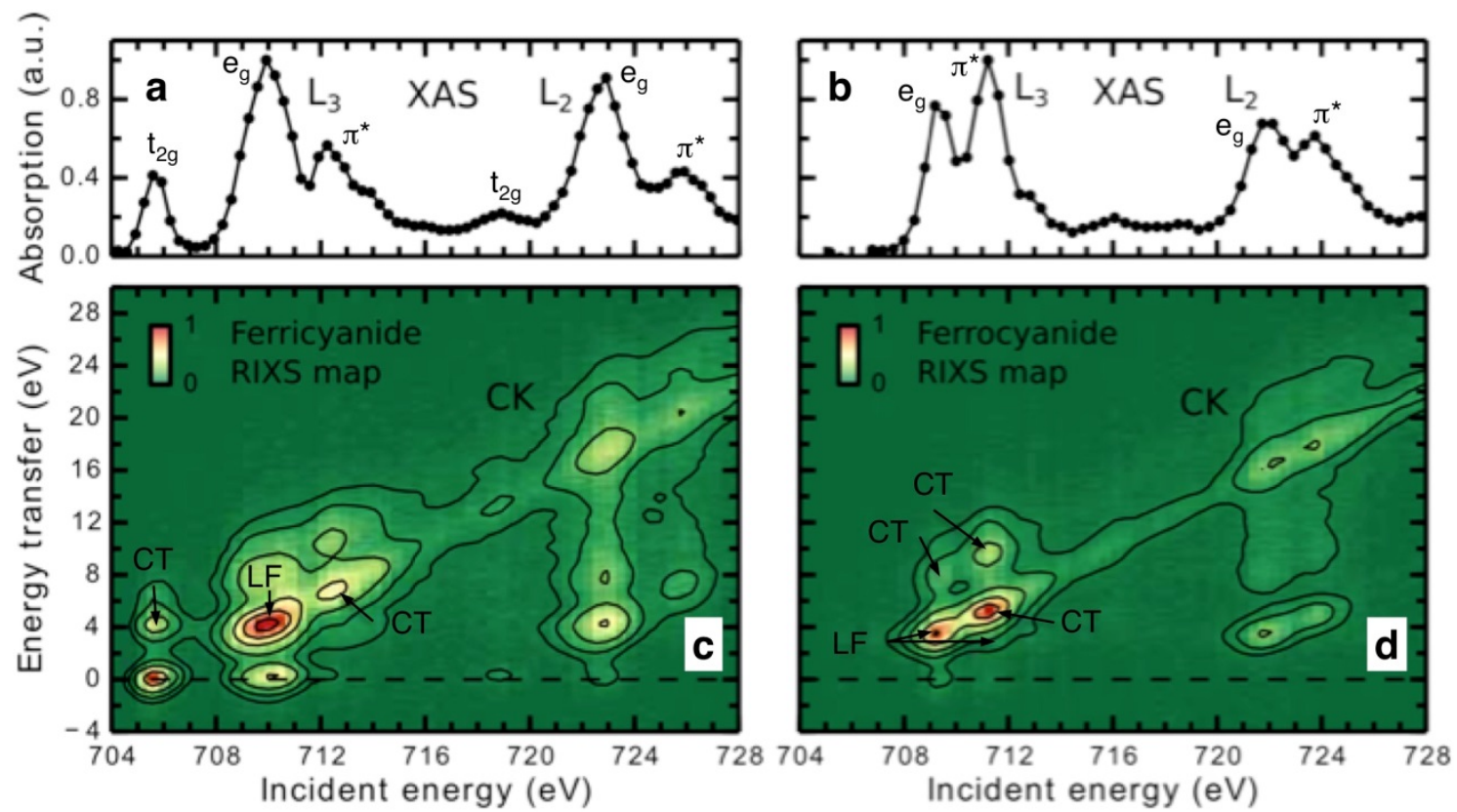

Figure 3. Metal L-edge spectroscopy of iron hexacyanides. L-edge XAS spectra of a ferri- and $\mathbf{b}$ ferrocyanide with approximate orbital assignments. Fe 2p3d RIXS maps of $\mathbf{c}$ ferri- and $\mathbf{d}$ ferrocyanide with approximate transition assignments. The spectral features labeled CK arise from so-called Coster-Kronig decays of the core-excited states at the $\mathrm{L}_{2}$-edge. All spectra are normalized to 1 at the maximum intensity. Adapted with permission from (Kunnus et al., 2016c). Copyright 2016 American Chemical Society.

Now looking at the RIXS maps in Figure $3 c$ and $3 d$, the spectral features can be categorized according to the final states that are reached and we proceed according to increasing incident photon energy. We focus here exclusively on the $L_{3}$ absorption edge and refer to the original publication for the discussion of the $L_{2}$ edge. Starting with the $t_{2 g}$ XAS peak in the $\mathrm{L}_{3}$-edge in ferricyanide, the RIXS peak at $0 \mathrm{eV}$ energy transfer results from elastic transitions where the $t_{2 g}$ electrons fill the $2 p$ hole (Figure $3 c$ ). Going to higher energy transfers a RIXS peak appears at $4 \mathrm{eV}$. This must correspond to emission from filled lower-lying orbitals and theoretical simulations indicate that these are the filled ligand-dominated $\sigma$ orbitals, which participate in $\sigma$ donor bonding. The RIXS peak at $4 \mathrm{eV}$ is thus a CT transition (specifically a ligand-to-metal charge-transfer, LMCT, transition), here observed using element-selective $x$ ray spectroscopy. In the RIXS spectrum this transition is well separated from the LF transitions as well as from other CT transitions such as metal-to-ligand charge-transfer (MLCT) transitions. Often such transitions partly overlap in the UV/Vis spectrum because of similar final-state energies. 
Proceeding further to the $e_{g}$ XAS peak and with electrons in filled ligand-dominated orbitals, as well as $t_{2}$ and $e_{g}$, several emission channels for filling the $2 p$ hole are open. The most intense transition is $t_{2 g} \rightarrow 2 p$, which gives final states that correspond to $t_{2 g} \rightarrow e_{g} L F$ transitions close to $4 \mathrm{eV}$, see Figure 3c. In contrast to UV/Vis experiments, where ligand-field transitions gain intensity only through vibronic coupling due to symmetry distortions, these LF excitations are actually the most intense transition in the RIXS map. This is due to the differences between the single-photon (UV/Vis) and the two-photon (RIXS) experiments. In the former case, LF transitions are forbidden because they have constant parity and orbital angular momentum, but this no longer holds when two photons are involved such as in RIXS. These differences in selection rules lead to relative enhancement of the LF transitions in RIXS compared to UV/Vis.

The ferricyanide $e_{g}$ XAS peak is broad in the energy transfer direction extending over the energy transfer range of 2-8 eV (Figure 3c) because it contains several final states arising from $t_{2 g} \rightarrow e_{g}$ transitions with the same nominal $t_{2 g}{ }^{4} e_{g}{ }^{1}$ orbital occupation but different energies. This is explained with Figure 4. The strong $2 p$ spin-orbit coupling in the intermediate states breaks the spin-selection rules and allows for intense transitions to both doublet and quartet final states from the doublet ground state. This aspect is detailed below in the section on ligand-field excitations in metal complexes and nanoparticles. In the iron hexacyanides, in addition, states split because of differences in electron-electron repulsion. For the ferricyanide $e_{g}$ XAS peak, the key factor is whether the single $e_{g}$ electron is in the same plane as the fourth $t_{2 g}$ electron $\left(d(x y)-d\left(x^{2}-y^{2}\right) T_{2 g}\right)$ or in different planes $\left(d(x y)-d\left(z^{2}\right) T_{1 g}\right)$ (Figure 4). The latter is lower in energy because it minimizes Coulomb repulsion. The energy difference between states is larger if the $3 d$ orbitals are localized on the metal, i.e. if the orbital covalency is low. Covalency in the sense discussed here relates to the degree of covalent orbital mixing between metal $3 d$ and water orbitals and the less covalent the bond, the larger the metal $3 d$ character and the smaller the ligand character of the metal-ligand orbitals. RIXS is typically able to observe both these states, and if they can be separated with high enough spectral resolution, this can give detailed information about metal-ligand interactions as will be discussed for hard $x$-ray RIXS below. Thus, the $t_{2 g} \rightarrow e_{g}$ resonance is not only enhanced in RIXS but multiple states are reached that, if they can be resolved, measure spin-state splittings and metal-ligand interactions. 


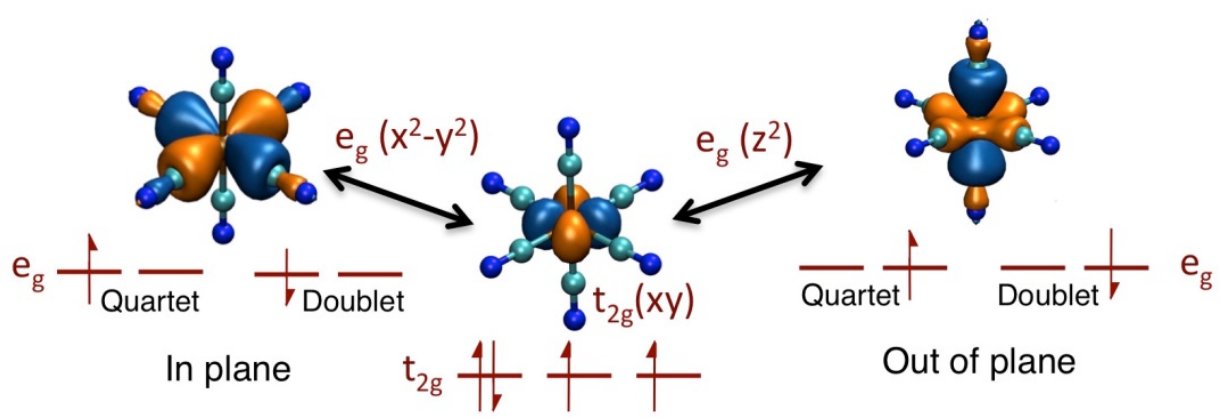

Figure 4. Schematic illustrations of doublet and quartet ${ }^{2,4}\left(T_{2 g}, T_{1 g}\right)$ valence-excited states with $t_{2 g}{ }^{4} e_{g}{ }^{1}$ configuration (drawn separately for quartet and doublet) reached in the RIXS process from the doublet ${ }^{2} \mathrm{~T}_{2 \mathrm{~g}}$ ground state with $\mathrm{t}_{2 \mathrm{~g}}{ }^{5} \mathrm{e}_{\mathrm{g}}{ }^{0}$ configuration (with calculated orbitals).

Looking at the RIXS transitions at the XAS peak labeled $\pi^{*}$ in ferricyanide (Figure $3 c$ ), several RIXS transitions extending all the way up to energy transfers of $12 \mathrm{eV}$ and above can be observed. The most intense RIXS peak at an energy transfer of $7 \mathrm{eV}$ can be assigned to a $t_{2 g} \rightarrow \pi^{*}$ MLCT excitation. Again, the position in the RIXS map clearly separates this transition from other possible CT transitions in the same energy transfer region.

The RIXS map of ferrocyanide (Figure 3) contains similar information as that of ferricyanide. At the $e_{g}$ XAS resonance we find a weak elastic peak and a prominent region of LF excitations with $t_{2 g} \rightarrow e_{g}$ transitions with several states split by differences in spin multiplicity and electron repulsion as marked in Figure 4. There are also $\sigma \rightarrow \mathrm{e}_{\mathrm{g}} \mathrm{CT}$ transitions at higher energies in the same $e_{g}$ XAS resonance and CT excitations are found at the $\pi^{*}$ XAS peak and it is noteworthy again that, in contrast to UV/Vis, both CT transitions are well separated from the other transitions.

As a side note we mention the spectral features labeled CK for Coster-Kronig in the RIXS maps of ferri- and ferrocyanide in Figures $3 c$ and $3 d$ occurring at the $\mathrm{L}_{2}$-edge and at energy transfers of around $20 \mathrm{eV}$. These arise from so-called Coster-Kronig decays of the coreexcited states at the $\mathrm{L}_{2}$-edge (Gotz et al., 2012, Wernet et al., 2012) and may contain information on the metal-ligand covalency in transition meal complexes (Wang et al., 2017).

The iron hexacyanide spectra illustrate the advantages of RIXS for probing valence excitations in an element-selective way, showing intense metal-centered LF transitions also for centrosymmetric complexes that are forbidden in UV/Vis spectroscopy. Due to its importance, this aspect is detailed below in a separate section. The possibility to observe states with different symmetry and spin multiplicities, as well as clearly separating different 
charge-transfer transitions is a clear advantage of the multidimensional RIXS method. All these features make L-edge RIXS a powerful probe of metal-ligand bonding.

\section{Hard X-ray RIXS: Metal K edges}

For metal L-edge spectroscopy the significant solvent absorption in the soft x-ray region is particularly important when dealing with dilute samples, which makes it challenging to apply L-edge RIXS to low-concentration samples or metalloproteins. Dilute metal samples are instead typically probed using hard $\mathrm{x}$-rays where the direct probe of the metal $3 \mathrm{~d}$ orbitals is commonly done with the $1 \mathrm{~s} \rightarrow 3 \mathrm{~d}$ excitations at the metal $\mathrm{K}$ pre-edge. The main problem with metal $1 \mathrm{~s}$ holes is that the short lifetime of $1 \mathrm{~s}$ core-excited states leads to large broadenings on the order of $1 \mathrm{eV}$ (compared to the order of $0.1 \mathrm{eV}$ for $2 \mathrm{p}$ core-hole states) that obscure spectral information. An important advantage of RIXS compared to XAS now is that the lifetime broadening in the energy transfer direction only depends on the lifetime of the RIXS final states. When core-excited final states are reached in K-edge RIXS such as $2 p$ core-hole states in $1 s 2 p$ RIXS or $3 p$ core-hole states in 1s3p RIXS (Figure 1b) the lifetime broadening of these core-hole states affects and often limits the highest-possible achievable spectral resolution in the energy-transfer direction in the RXIS spectra. Often the choice of a particular emission channel in K-edge RIXS ( $K \alpha$ in $1 s 2 p$ RIXS or $K \beta$ in $1 s 3 p$ RIXS) thus selects both the final-state interactions that are probed ( $2 p-3 d$ in $1 s 2 p$ RIXS and $3 p-3 d$ in $1 s 3 p$ RIXS) and the spectral resolution that can be obtained. Although a resolution of below $0.1 \mathrm{eV}$ has been achieved in hard $x$-ray RIXS in the investigation of low-energy excitations in magnetic materials and superconductors, (Forte et al., 2008, Ament et al., 2011), in many chemistry applications it is more important to get higher detection efficiency at more moderate resolutions. In L-edge RIXS, for comparison, the lifetime of the valence-excited final states is on the order of nanoseconds and longer and the lifetime broadening of these can often be neglected (compared to the experimental bandwidth or inhomogeneous broadening).

If the target is to probe dilute systems in a solvent with K-edge RIXS, the logical choice is the strongest emission channel $K \alpha(2 p \rightarrow 1 s$ transitions). The final state in this $1 s 2 p$ RIXS process has an orbital occupation of $2 p^{5} 3 d^{n+1}$, where $n$ is the number of $3 d$ electrons in the ground state (Figure 1b). This is the same final-state configuration as in L-edge XAS. The energy transfer in the $1 \mathrm{~s} 2 \mathrm{p}$ RIXS experiment thus corresponds to the direct L-edge absorption 
energy. This will be detailed below in this section for the example of the iron hexacyanides also showing that RIXS has significant advantages compared to the direct absorption. $1 \mathrm{~s} 2 \mathrm{p}$ RIXS data have been collected for several enzymes and solution catalysts including photosystem II and cytochrome c (Glatzel and Bergmann, 2005, Glatzel et al., 2004, Glatzel et al., 2013, Leidel et al., 2012, Kroll et al., 2014), where the latter will be discussed in more detail in the section on the applications of metal K-edge RIXS.

The basic principle of a $1 s 2 p$ RIXS experiment is again illustrated using the two iron hexacyanide complexes. Fe 1s2p RIXS data for these complexes have been collected both in the solid state and, for direct comparison to L-edge XAS, in solution (Lundberg et al., 2013, Penfold et al., 2014). Here and in order to highlight the sensitivity to chemical bonding, the Fe $1 s 2 p$ RIXS spectra of the study on solid samples are compared to two complexes with the same formal oxidation state and spin multiplicity, ferrous tacn $\left[\mathrm{Fe}^{\prime \prime}(\operatorname{tacn})_{2}\right]^{2+}(\operatorname{tacn}=1,4,7$ triazacyclononane) and ferric tacn $\left[\mathrm{Fe}^{\prime \prime \prime}(\operatorname{tacn})_{2}\right]^{3+}$ (Lundberg et al., 2013). The main difference between the complexes is that tacn is mainly a $\sigma$ donor ligand but with weaker $\sigma$ covalency. In both ferrous tacn and ferrocyanide the initial excitation in $\mathrm{K}$ pre-edge XAS from the $\mathrm{t}_{2 \mathrm{~g}}{ }^{6} \mathrm{e}_{\mathrm{g}}{ }^{0}$ ground state is a $1 \mathrm{~s} \rightarrow \mathrm{e}_{\mathrm{g}}$ transition at $7111 \mathrm{eV}$ for ferrous tacn and $7113 \mathrm{eV}$ for ferrocyanide, and there are no other transitions below the rising K-edge, see Figure 5. The K pre-edge XAS alone thus gives very limited information about the electronic structure. However, the RIXS spectra of the two complexes show clear differences along the energy transfer direction, with the first RIXS peak in ferrous tacn (706-708 eV, Figure 5a) clearly being wider, due to a two-peak structure, than the first RIXS peak in the ferrocyanide spectrum (709 eV, Figure $5 b)$.
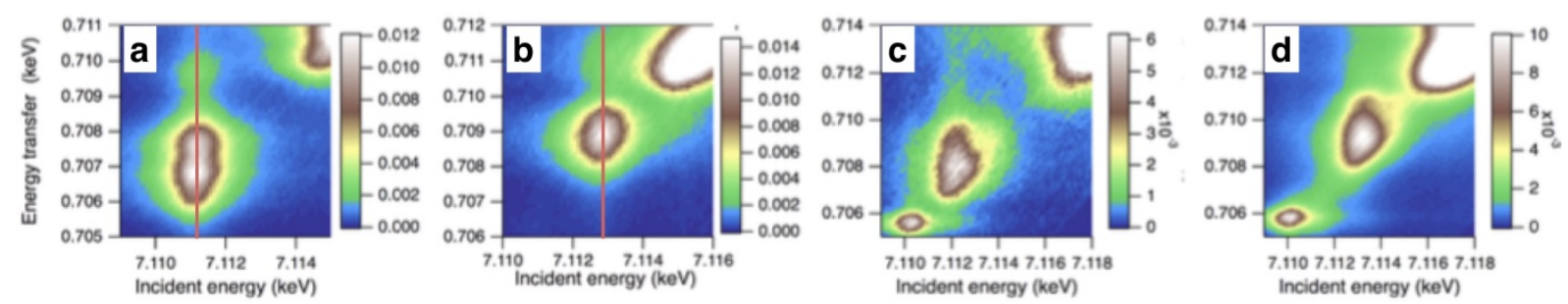

Figure 5. Experimental Fe 1s $2 \mathrm{p}$ RIXS planes of a ferrous tacn $\left[\mathrm{Fe}^{\prime \prime}(\operatorname{tacn})_{2}\right] \mathrm{Br}_{2}$, b ferrocyanide $\left[\mathrm{Fe}^{\prime \prime}(\mathrm{CN})_{6}\right] \mathrm{K}_{4}, \mathbf{c}$ ferric tacn [ $\left.\mathrm{Fe}^{\prime \prime \prime}(\operatorname{tacn})_{2}\right] \mathrm{Br}_{3}$ and $\mathbf{d}$ ferricyanide $\left[\mathrm{Fe}^{\prime \prime \prime}(\mathrm{CN})_{6}\right] \mathrm{K}_{3}$. Only the energy-transfer ranges corresponding to the $\mathrm{L}_{3}$-edge are shown (at higher incident energies of $>7114 \mathrm{eV}$ and $>710 \mathrm{eV}$ the rising K-edge dominates the spectral shape). Constant incident energy (CIE) cuts as discussed in the text are shown in $\mathbf{a}$ and $\mathbf{b}$. Adapted with permission from (Lundberg et al., 2013). Copyright 2013 American Chemical Society. 
To analyze the differences further it is instructive to compare the ferrous $1 s 2 p$ RIXS spectra of Figure 5 to the corresponding L-edge XAS spectra and this is done in Figure 6 (the ferrocyanide L-edge XAS spectrum is displayed in Figure 3b). As the energy transfer in the $1 s 2 p$ RIXS experiment at a given incident energy corresponds to L-edge absorption energies L-edge-like absorption spectra can be obtained by taking vertical cuts along the energy transfer axis with constant incident energy (CIE), shown as red lines in Figures $5 a$ and $5 b$ (Glatzel and Bergmann, 2005). One could anticipate that with a single dominant K pre-edge transition, the CIE cut should access almost all $2 p^{5} 3 d^{7}$ states in the ferrous systems and directly correspond to the L-edge XAS spectrum. However, the direct comparison shows significant differences between the 1s2p RIXS CIE cut and the L-edge XAS spectrum for both ferrous tacn and ferrocyanide, see Figure 6 . The first peaks in the $L_{3}$-edges, assigned to $2 p \rightarrow e_{g}$ transitions are wider in the $1 s 2 p$ RIXS CIE cut than in the L-edge XAS spectrum, and this increase in width gives important information about electronic structure as described in detail below. Further, as the comparison in Figure $6 \mathrm{~b}$ shows, the $\pi^{*}$ peak that appears as a second strong peak at the $\mathrm{L}_{3}$-edge in the ferrocyanide XAS spectrum is largely missing in the $1 s 2 p$ RIXS CIE cut. This shows that $1 s 2 p$ RIXS, despite all its advantages, is a less sensitive probe of $\pi$ backbonding than L-edge XAS.
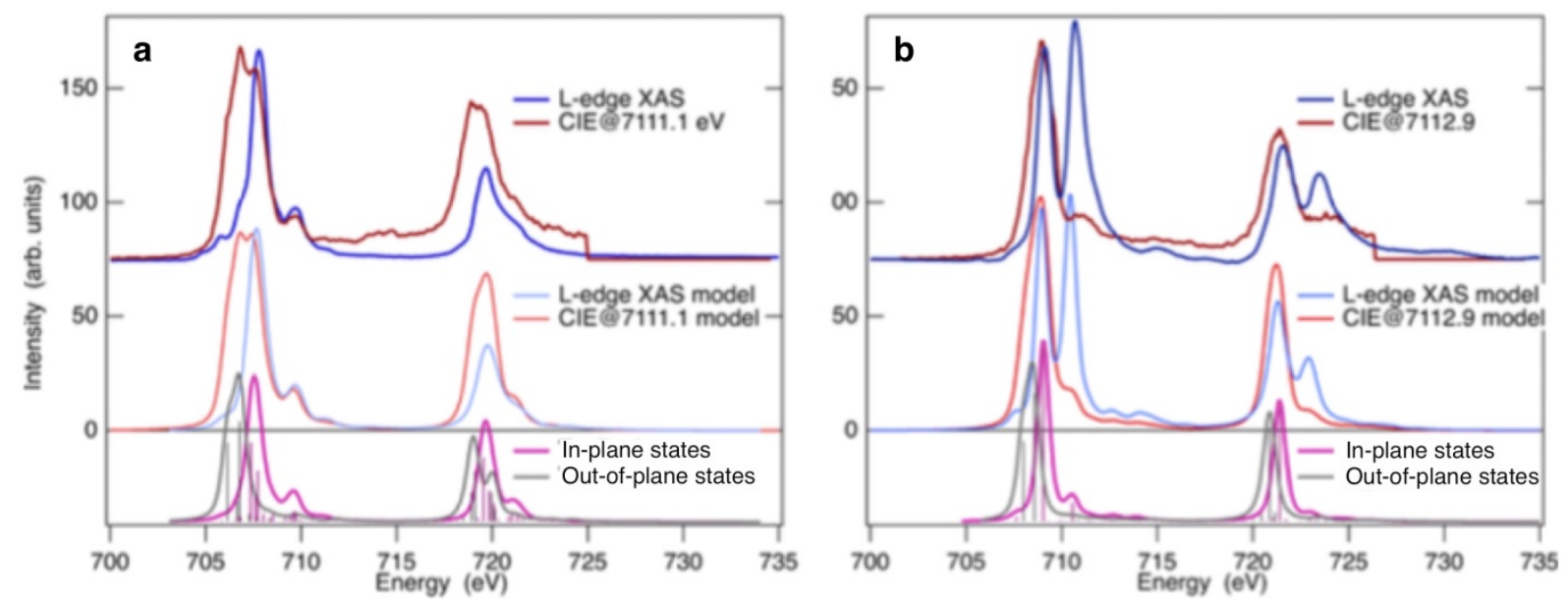

Figure 6. Comparison of the Fe 1s2p RIXS constant incident energy (CIE) cuts through the maximum of the $\mathrm{K}$ pre-edge resonance (dark red) to the L-edge XAS spectrum (dark blue) of a ferrous tacn and $\mathbf{b}$ ferrocyanide. The results from charge-transfer multiplet (CTM) model calculations are shown below the experimental results for both CIE cut (light red) and L-edge XAS (light blue). Separate contributions to the RIXS CIE spectra from final states with in-plane and out-of-plane alignment are shown in purple and gray, respectively. Adapted with permission from (Lundberg et al., 2013). Copyright 2013 American Chemical Society. 
Theoretical analyses of $1 s 2 p$ RIXS and L-edge XAS using the charge-transfer multiplet (CTM) model, show that the increase in width of the first peak in the $L_{3}$-edge in $1 s 2 p$ RIXS is due to new low-energy states that are allowed in the RIXS experiment, but forbidden in L-edge XAS (Lundberg et al., 2013, Guo et al., 2016). The energy difference between the states can largely be explained using the same picture as for the splitting of the valence states in Figure 4. Ignoring complications from spin-orbit coupling, the low-energy states have the $2 p$ hole oriented in the same plane as the $e_{g}$ electron, e.g., as a $2 p_{z}$ hole and a $3 d\left(z^{2}\right)$ electron. The high-energy states have the $2 p$ hole out of plane relative to the $e_{g}$ electron, e.g., as a $2 p_{z}$ hole and a $3 d\left(x^{2}-y^{2}\right)$ electron. The in-plane states are favored because they increase the attractive interaction between the $2 p$ hole and the $3 d$ electron and they are allowed in twophoton 1s2p RIXS adding intensity at low energies. These states, however, are not allowed in single-photon L-edge XAS and are therefore missing in this spectrum.

Beyond just a simple illustration of the different selection rules of $1 s 2 p$ RIXS and L-edge XAS, the width of the RIXS CIE cut gives in addition direct information about the covalency of the $\mathrm{e}_{\mathrm{g}}$ orbital. Comparing the ferrous tacn and the ferrocyanide RIXS spectra in Figures $5 \mathrm{a}$ and $5 \mathrm{~b}$ it is clear that the width along the energy transfer direction (red lines in Figures $5 \mathrm{a}$ and $5 \mathrm{~b}$ ) is not the same in the two complexes. This is also obvious from comparing the $1 \mathrm{~s} 2 \mathrm{p}$ RIXS CIE cuts in Figures 6a and 6b: Apparently the extracted $\mathrm{L}_{3}$-edge is considerably wider in ferrous tacn compared to ferrocyanide. This difference in width is due to differences in the interactions between the localized $2 p$ hole and the $e_{g}$ electrons in different orientations. These interactions are smaller in case the $e_{g}$ orbital is delocalized over the ligands, i.e. for the more covalent system. Directly from the $1 s 2 p$ RIXS spectra it is thus clear that the $e_{g}$ orbital is more covalent in ferrocyanide compared to ferrous tacn. More quantitative estimates of metal-ligand covalencies can be extracted from more advanced theoretical treatments such as from the CTM model.

The comparison of the ferric $1 \mathrm{~s} 2 \mathrm{p}$ RIXS and L-edge XAS spectra is more complicated (Figures 5 and 3 ). As for the L-edges, the $K$ pre-edge consists of a $t_{2 g}$ XAS resonance at lower and an $e_{g}$ resonance at higher incident energies (Figure 5). The significant differences in shape of the two resonances indicate how sensitive the K-edge RIXS spectra are to the electronic structure. The $t_{2 g}$ resonance is sharp in both incident and energy transfer directions (Figure 5), which signals that this is a closed $\mathrm{t}_{2 \mathrm{~g}}{ }^{6} \mathrm{e}_{\mathrm{g}}{ }^{0}$ valence shell where neither intermediate nor final states split in energy. The $e_{g}$ resonance instead leads to $t_{2 g}{ }^{5} e_{g}{ }^{1}$ that splits into four 
different absorption resonances, with parallel/antiparallel spin and in-plane/out-of-plane interactions similar to what was described for the L-edge XAS final states of ferrocyanide. The energy transfer direction is more complicated to analyze because of the additional core hole interactions that further split these states. These splittings are the origin for the broader 1s2p RIXS CIE cuts compared to the L-edge XAS spectra in a similar way as shown for ferrocyanide in Figure 6a.

The 1s $2 p$ RIXS spectra of ferro- and ferricyanide show that the experiment, in addition to the advantages of the hard $\mathrm{x}$-ray probe, provides additional information compared to the singlephoton L-edge XAS experiment that can be used to extract detailed electronic structure information. However, in many cases the spectra are complex and require a theoretical model to correlate spectra and metal-ligand bonding characteristics. To further direct this analysis, it is fruitful to compare complexes with similar electronic structure. These lessons will be illustrated in the discussion of cytochrome c below.

$K \beta$ RIXS (Figure 1b) gives access to a wide range of final states, all the way from those with $3 p$ core holes (with $K \beta_{1,3}$ and $K \beta^{\prime}$ emissions for $1 s 3 p$ RIXS) to valence-excited states (with $K \beta_{2,5}$ and $K \beta^{\prime \prime}$ emissions for VTC RIXS). The drawback when using $K \beta$ emission for $1 s 3 p$ RIXS instead of $\mathrm{K} \alpha$ emission in $1 \mathrm{~s} 2 \mathrm{p}$ RIXS is the loss of intensity because of the about ten times weaker $\mathrm{K} \beta_{1,3} / \mathrm{K} \beta^{\prime}$ emission compared to $\mathrm{K} \alpha$ emission (Glatzel and Bergmann, 2005). The technique has therefore mainly been applied to synthetic complexes and other highconcentration samples. Valence excitations with hard $\mathrm{x}$-rays have been studied for systems ranging from manganese hexacyanide (Meyer et al., 2010) to metal-organic frameworks (Gallo et al., 2017). In the section on applications of metal K-edge RIXS we discuss how 1s3p RIXS can be used to understand the activity of a cobalt cubane of relevance for water oxidation (Hadt et al., 2016).

\section{Applications of Metal L-edge RIXS}

The idea for this section is to detail how 2 p3d RIXS can be used to provide a metal-centric view of the electronic structure of metal complexes and nanoparticles thereby complementing the information from UV/Vis spectroscopy and other $\mathrm{x}$-ray spectroscopic methods such as XAS, XES and XPS. One limitation of XAS, XES and XPS is that the strong 
interactions of the core hole in the final core-excited states can give complicated spectral shapes. It is therefore not necessarily straightforward to connect the spectral information from these methods to the electronic structure of the ground state of the system although this is the property of interest in many chemistry problems. A prominent example for this complication is the extraction of LF excitations in metal complexes from metal L-edge XAS or XPS. The final states of these methods are $2 p^{5}$ core-hole states and the influence of the corehole on the extracted ligand-field energies has been determined to several $10 \%$, an effect that needs to be accounted for in the analyses of spectra from XAS and XPS (De Groot and Kotani, 2008). Another limitation of XAS, XES and XPS is that often the short lifetime of the core-excited states gives rise to lifetime broadening in the spectra that can obscure spectral detail because the lifetime broadening is large compared to typical energy splitting of LF states. In this section we describe how these limitations of the more commonly used x-ray spectroscopy methods can be overcome with the use of RIXS.

Following pioneering work on probing LF excitations with L-edge RIXS in solid-state metal systems, (Butorin et al., 1996a, Kuiper et al., 1998), recent metal 2p3d RIXS investigations of LF excitations in metal complexes are discussed. In 2p3d RIXS the LF excitations are dipole allowed and it is shown how they are hence strong compared to CT excitations (van Schooneveld et al., 2013a). In addition, examples are presented illustrating that LF transitions, that are spin-forbidden in UV/Vis absorption, are allowed in 2p3d RIXS due to the strong metal $2 p$ spin-orbit interaction in the intermediate core-excited states (Hahn et al., 2017, Van Kuiken et al., 2016). Further, it is demonstrated that if the 2p3d RIXS measurement is performed with sufficient resolution the important energy range below 0.5 $\mathrm{eV}$ can be readily accessed. Finally, very recent proposals are presented for how information on the metal-ligand covalency in metal complexes can be extracted from CT excitations in 2p3d RIXS.

\section{Ligand-field excitations in metal complexes and nanoparticles}

We start with the example of a simple ionic system studied by Kunnus et al. (Figure 7) to establish the concept of probing LF excitations with 2p3d RIXS and to link this to the 3dimensional RIXS measurements of scattered intensities versus incident energy and energy transfer as introduced in the first section of this chapter (Kunnus et al., 2013). 

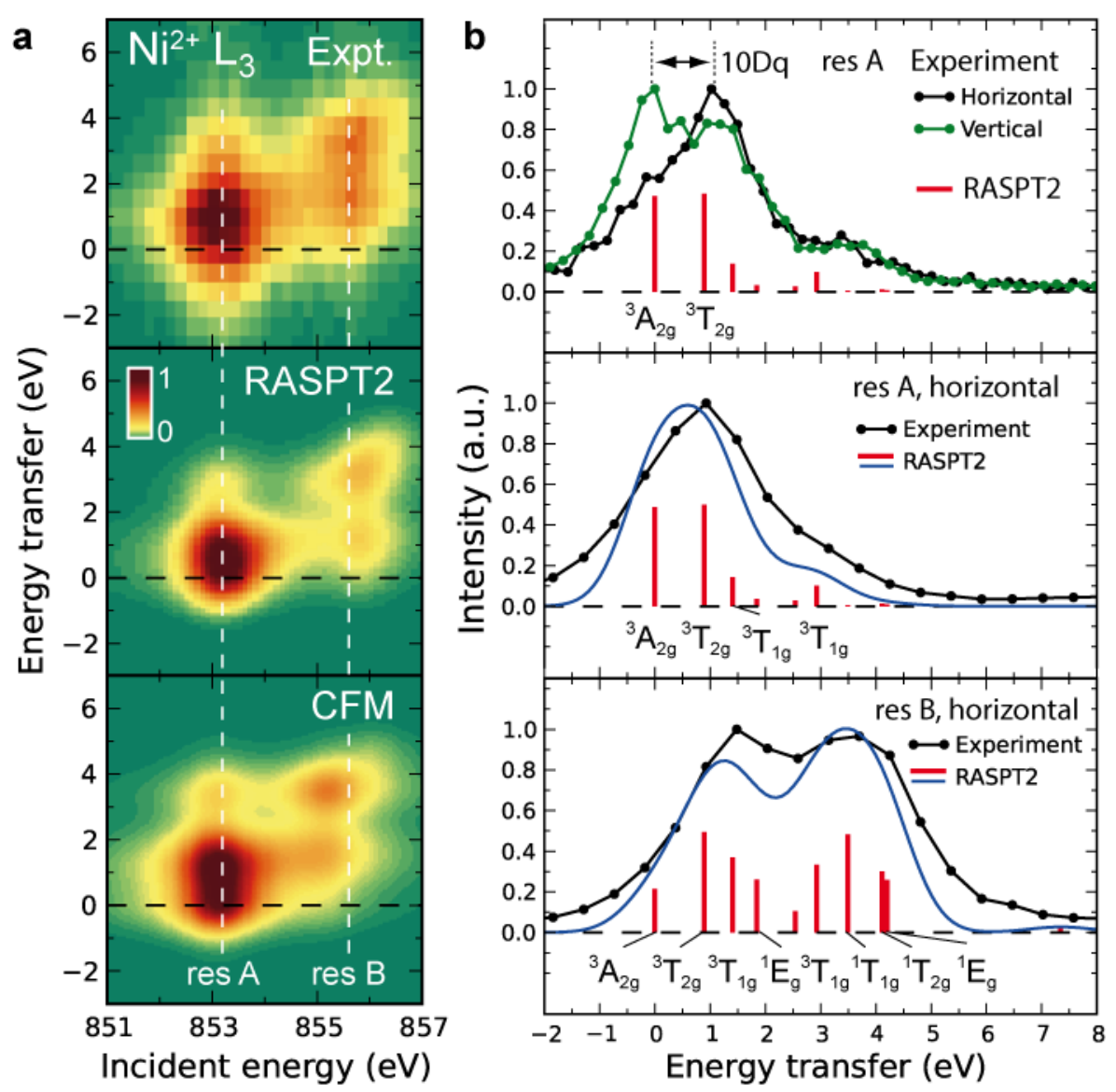

Figure 7. a Measured (Expt.) and calculated (RASPT2 and CFM) Ni $\mathrm{L}_{3}$-edge RIXS maps of $\mathrm{Ni}^{\prime \prime}$ in $\mathrm{O}_{\mathrm{h}}$ symmetry $\left(\mathrm{Ni}^{\mathrm{II}}\right.$ in $\left[\mathrm{Ni}\left(\mathrm{H}_{2} \mathrm{O}\right)_{6}\right]^{2+}$ from $\mathrm{NiCl}_{2}$ in aqueous solution, RASPT2 are restricted active space and CFM are crystal-field multiplet calculations, intensities encoded in color and normalized to one at maximum, experimental bandwidth $1.2 \mathrm{eV}$ ). b Vertical cuts ( $\mathrm{Ni}_{3}$-RIXS spectra, normalized to one at maximum) through the RIXS maps in a (black/green: experiment, blue/red: RASPT2 calculations, incident energies $853.2 \mathrm{eV}$ : resonance $\mathrm{A}$, top and middle, and $855.6 \mathrm{eV}$ : resonance $\mathrm{B}$, bottom). Red sticks are calculated RASPT2 ligand-field peaks underlying the convoluted lines spectra. Top panel: Experimental RIXS spectra are shown for different polarizations of the incident radiation (for horizontal/vertical the polarization vector is parallel/perpendicular to the RIXS scattering plane of the experiment). Adapted with permission from (Kunnus et al., 2013). Copyright 2013 American Chemical Society.

Figure 7 shows the Ni 2p3d RIXS maps and ligand-field spectra of $\mathrm{Ni}^{\text {Il }}$ in the octahedral aqueous complex $\left[\mathrm{Ni}\left(\mathrm{H}_{2} \mathrm{O}\right)_{6}\right]^{2+}$ as prepared in a liquid jet in vacuum. $\mathrm{Ni}^{11}$ ions in aqueous solution exhibit comparably weak covalent interactions of the $\mathrm{Ni}$ il ion with the water solvent molecules of the first hydration sphere. The measured Ni 2p3d RIXS maps can therefore be described both with restricted active space (RAS) calculations treating orbital interactions explicitly and with the crystal-field multiplet (CFM) model that excludes metal-ligand charge transfer (Figure 7a). The RIXS maps in Figure 7a (only the $\mathrm{Ni}_{3}$-edge is shown) exhibit two XAS resonances $A$ and $B$ and RIXS intensities arising from LF excitations at energy transfers of 
0-3 eV at resonance $A$ and $0-5 \mathrm{eV}$ at resonance $\mathrm{B}$. $\mathrm{Ni}^{\mathrm{II}}-\mathrm{O}_{\mathrm{h}}$ in $\left[\mathrm{Ni}\left(\mathrm{H}_{2} \mathrm{O}\right)_{6}\right]^{2+}$ with a nominal $3 \mathrm{~d}^{8}$ electron configuration has a ${ }^{3} \mathrm{~A}_{2 \mathrm{~g}}$ ground state with a valence-electron configuration $\left(\mathrm{Le}_{\mathrm{g}}, \mathrm{Lt} \mathrm{Tg}^{10}\left(\mathrm{t}_{2 \mathrm{~g}}\right)^{6}\left(\mathrm{e}_{\mathrm{g}}\right)^{2}\right.$ where $\mathrm{Le}_{\mathrm{g}}$ and $\mathrm{Lt} \mathrm{t}_{2 \mathrm{~g}}$ are the water-ligand dominated bonding orbitals and $e_{g}$ and $t_{2 g}$ are the Ni-dominated $3 d$ antibonding orbitals. Absorption of $\left[\mathrm{Ni}\left(\mathrm{H}_{2} \mathrm{O}\right)_{6}\right]^{2+}$ at the $\mathrm{Ni}$ $\mathrm{L}_{3}$-edge (Figure $7 \mathrm{a}$ ) can be attributed for both resonances $\mathrm{A}$ and $\mathrm{B}$ to $2 p \rightarrow \mathrm{e}_{\mathrm{g}}$ one-electron transitions to $2 \mathrm{p}^{5}\left(\mathrm{Le}_{\mathrm{g}}, \mathrm{Lt}_{2 \mathrm{~g}}\right)^{10}\left(\mathrm{t}_{2 \mathrm{~g}}\right)^{6}\left(\mathrm{e}_{\mathrm{g}}\right)^{3}$ intermediate core-excited states. Resonance $\mathrm{A}$ is dominated by transitions from the triplet ground state to triplet core-excited states with parallel spins of the unpaired $2 p$ and $e_{g}$ electrons (Josefsson et al., 2012). Resonance $B$ in contrast corresponds to transitions to both singlet and triplet core-excited states where the singlet states have antiparallel spins of the unpaired $2 p$ and $e_{g}$ electrons (Josefsson et al., 2012). Ni 2p3d RIXS at these resonances results in the ligand-field spectra in Figure 7b and corresponds to displaying vertical cuts through the maps in Figure $7 \mathrm{a}$ at the incident energies of maximal intensities at the two XAS resonances. The LF excitations of this complex thus involve only $\mathrm{Ni} t_{2 g}$ and $e_{g}$ orbitals and correspond to $t_{2 g} \rightarrow e_{g}$ transitions from the ${ }^{3} A_{2 g}$ $\left(\mathrm{Le}_{\mathrm{g}}, \mathrm{Lt} \mathrm{tg}_{\mathrm{g}}\right)^{10}\left(\mathrm{t}_{2 \mathrm{~g}}\right)^{6}\left(\mathrm{e}_{\mathrm{g}}\right)^{2}$ ground state to $\left(\mathrm{Le}_{\mathrm{g}}, \mathrm{Lt} \mathrm{t}_{2 \mathrm{~g}}\right)^{10}\left(\mathrm{t}_{2 \mathrm{~g}}\right)^{5}\left(\mathrm{e}_{\mathrm{g}}\right)^{3}$ and $\left(\mathrm{Le}_{\mathrm{g}}, \mathrm{Lt}_{2 \mathrm{~g}}\right)^{10}\left(\mathrm{t}_{2 \mathrm{~g}}\right)^{4}\left(\mathrm{e}_{\mathrm{g}}\right)^{4}$ final states. The calculated transitions are displayed as sticks (separate transitions) and convoluted line spectra in Figure $7 b$ (the peak labelled ${ }^{3} \mathrm{~A}_{2 \mathrm{~g}}$ corresponds to elastic scattering back to the ground state). We note that focusing on the $\mathrm{L}_{3}$-edge only and discarding the $\mathrm{L}_{2}$-edge is justified here and in many metal complexes in general as often the same final RIXS states are reached. Comparison of the measured and calculated $\mathrm{Ni} 2 \mathrm{p} 3 \mathrm{~d}$ RIXS spectra of $\left[\mathrm{Ni}\left(\mathrm{H}_{2} \mathrm{O}\right)_{6}\right]^{2+}$ in Figure $6 \mathrm{~b}$ shows that triplet ligand-field states ${ }^{3} \mathrm{~A}$ and ${ }^{3} \mathrm{~T}$ at $0-3 \mathrm{eV}$ are probed at resonance $\mathrm{A}$ via the triplet intermediate core-excited states while at resonance B singlet ligand-field states ${ }^{1} \mathrm{E}$ and ${ }^{1} \mathrm{~T}$ up to $5 \mathrm{eV}$ are strong as well due to transition through the mixed singlettriplet intermediate core-excited states at resonance $B$. This is a clear manifestation of probing nominally spin-forbidden LF excitations with 2 p3d RIXS. It also shows how, by tuning the incident photon energy to different resonances (different intermediate states) different final states can be resonantly enhanced. This example therefore demonstrates how $2 \mathrm{p} 3 \mathrm{~d}$ RIXS complements UV/Vis spectroscopy by probing nominally spin-forbidden LF transitions in metal complexes and resonantly enhancing certain final states.

As a last point for this example we discuss a trick that was used here to further enhance the information content of the $2 p 3 d$ RIXS measurement by making use of the polarization of the incident radiation. In the top panel of Figure $7 \mathrm{~b}$ the two measured RIXS spectra were taken 
with two perpendicular polarizations of the linearly polarized incident radiation (horizontal/vertical polarization corresponds to the polarization vector of the incident radiation being parallel/perpendicular to the scattering plane which is defined by the propagation directions of incident and scattered photons). Horizontal polarization is the most commonly used symmetry in $2 \mathrm{p} 3 \mathrm{~d}$ RIXS experiments and corresponds to the experimental realizations of all experiments discussed in this chapter (common to all is also the so-called " $90^{\circ}$ scattering geometry" which means that the angle between the propagation directions of incident scattered photons equals or is close to $90^{\circ}$ ). Vertical polarization enhances elastic scattering as can be understood by the analogy to the basic concept of the dipole antenna (also called the Hertzian dipole). In the spectra in Figure $7 \mathrm{~b}$ the enhancement of elastic scattering is apparent in the strong ${ }^{3} \mathrm{~A}_{2 \mathrm{~g}}$ peak at an energy transfer of $0 \mathrm{eV}$ in the vertical spectrum (the calculations are shown for horizontal polarization only). This trick facilitates determination of the ligand-field energy 10Dq, being the separation between the two lowest-lying states, of the $\left[\mathrm{Ni}\left(\mathrm{H}_{2} \mathrm{O}\right)_{6}\right]^{2+}$ complex to 1.1. eV as the ${ }^{3} \mathrm{~A}_{2 \mathrm{~g}^{-}}{ }^{3} \mathrm{~T}_{2 \mathrm{~g}}$ energy separation can be more easily extracted from the spectra.

The comparison of measured and calculated Ni 2p3d RIXS spectra in Figure $7 \mathrm{~b}$ also shows that for the $\left[\mathrm{Ni}\left(\mathrm{H}_{2} \mathrm{O}\right)_{6}\right]^{2+}$ complex considered here and for metal complexes in general a higher spectral resolution is desirable. With a bandwidth on the order of $0.1 \mathrm{eV}$ (instead of the $1 \mathrm{eV}$ used for the data in Figure $7 \mathrm{~b}$ ) more detailed insight would be evidently gained because the bandwidth would correspond to or be below the energetic splitting of the LF transitions in the range of tens of meV. It is the purpose of the following three examples to illustrate how high spectral resolution in metal 2p3d RIXS gives access to ligand coordination, ligand-field symmetry and symmetry distortions of the metal complexes.

The effect of ligand coordination and symmetry in 2p3d RIXS is illustrated with the seminal investigation by van Schooneveld et al. with the Co L-edge RIXS spectra of a series of solid Co" compounds in Figure 8 (van Schooneveld et al., 2013a). 


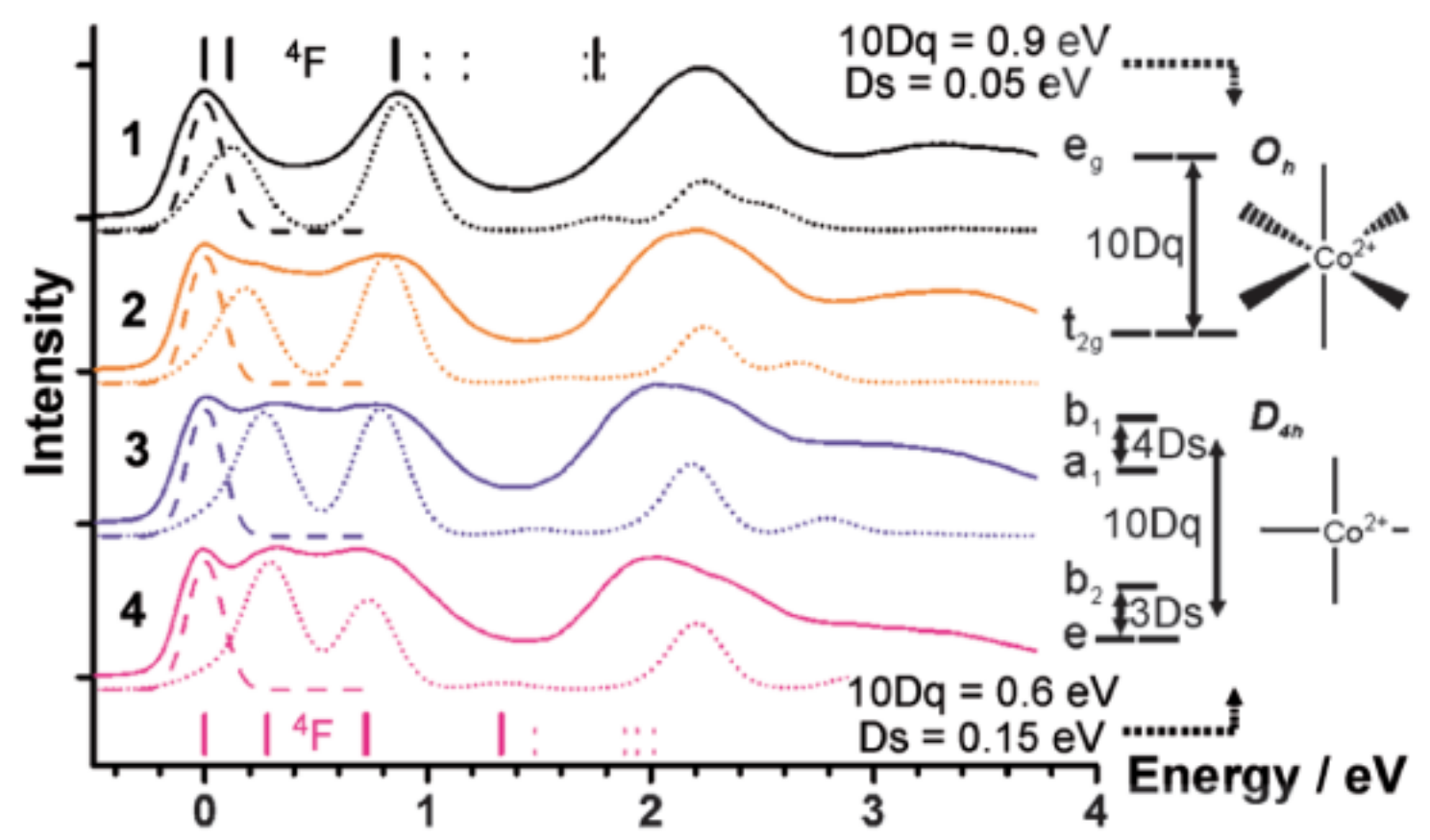

Figure 8. Co $\mathrm{L}_{3}$-RIXS spectra of $\mathrm{Co}^{\prime \prime}$ in $\mathrm{O}_{\mathrm{h}}$ and $\mathrm{D}_{4 \mathrm{~h}}$ symmetries for Co diformate dehydrate, $\left[\mathrm{Co}(\mathrm{HCOO})_{2}\left(\mathrm{H}_{2} \mathrm{O}\right)_{2}\right](1)$, Co diacetate, $\left[\mathrm{Co}\left(\mathrm{H}_{3} \mathrm{CCOO}\right)_{2}\left(\mathrm{H}_{2} \mathrm{O}\right)_{3}\right](2)$, Co dibenzoate, $\left[\mathrm{Co}\left(\mathrm{H}_{5} \mathrm{C}_{6} \mathrm{COO}\right)_{2}\right](3)$, and Co dioleate, $\left[\mathrm{Co}\left(\mathrm{H}_{33} \mathrm{C}_{17} \mathrm{COO}\right)_{2}\right]+\mathrm{H}_{33} \mathrm{C}_{17} \mathrm{COOH}$ ) (4), as measured (solid) and calculated (dotted, crystalfield multiplet, $\mathrm{CFM}$, calculations, atomic ${ }^{4} \mathrm{~F}$ multiplet energies as split by the given ligand fields are given as solid sticks for 1 and 4). The dashed Gaussians centered at $0 \mathrm{eV}$ represent the experimental bandwidth of $0.1 \mathrm{eV}$. Schematic molecular-orbital energy diagrams with crystal-field parameters Dq and Ds and sketches of $\mathrm{Co}^{\prime \prime}$ coordination in $\mathrm{O}_{h}$ and $\mathrm{D}_{4 h}$ symmetries are given on the right. Reproduced from (van Schooneveld et al., 2013a) with permission from John Wiley and Sons.

The spectra were taken at the Co $\mathrm{L}_{3}$ absorption resonance and demonstrate how Co $2 \mathrm{p} 3 \mathrm{~d}$ RIXS reflects the ligand-field changes associated with changing the average Co" coordination number from 6 (sample 1 in Figure 8) to 4 (sample 4 in Figure 8). Although not further emphasized it is important to realize that the element-specific resonant excitation at the Co $\mathrm{L}_{3}$-edge is the foundation for the analysis of the Co $2 p 3 d$ RIXS in terms of ligand-field energies locally at the Co" site. All studied compounds exhibit a ${ }^{4} A_{2 g}$ ground state (the corresponding atomic multiplet is ${ }^{4} \mathrm{~F}$ ) and the LF excitations at $0-3 \mathrm{eV}$ in Figure 8 as probed by Co L-edge RIXS are due to spin-preserving transitions from the ground state to ${ }^{4} E_{g},{ }^{4} B_{2 g}$, and ${ }^{4} \mathrm{~A}_{2 \mathrm{~g}}$ final states. The spectra were taken with an experimental bandwidth of $0.1 \mathrm{eV}$ (Gaussian FWHM, see dashed Gaussian at $0 \mathrm{eV}$ energy transfer in Figure 8) and this gives access to the observed spectral changes in the important energy range of 0-1 eV (we discard the peak at 2-2.5 eV here). Apparently, the measured Co 2p3d RIXS spectra form a two-peak spectrum with peaks close to 0 and $1 \mathrm{eV}$ for sample 1 (average Co" coordination 6) and change towards a spectrum with a more continuous intensity distribution between 0 and 1 
eV for sample 4 (average Co" coordination 4). The measured spectra are well described by spectra calculated within the CFM model and this comparison yields the quantitative information about ligand coordination, ligand-fields and symmetry based on the molecularorbital energy diagrams and molecular symmetries in Figure 8. The CFM model comprises calculated transitions from the ground to all allowed LF final states in the given symmetry. The crystal-field parameters $10 \mathrm{Dq}$ and Ds (Figure 8) were varied such that the resulting convoluted line spectra (dotted lines in Figure 8) best fit the experiment. Because the variation of LF transition energies with ligand-field parameters is effectively described with so-called Tanabe-Sugano diagrams (De Groot and Kotani, 2008), this approach of extracting LF parameters can be regarded as "fitting the Tanabe-Sugano diagram to the 2 p3d RIXS spectra" (van Schooneveld et al., 2013a). van Schooneveld et al. find best agreement for the investigated series for a decrease of 10Dq from $0.9 \mathrm{eV}$ (sample 1) to $0.6 \mathrm{eV}$ (sample 4) and a corresponding increase of Ds from $0.05 \mathrm{eV}(\mathbf{1})$ to $0.15 \mathrm{eV}(\mathbf{4})$. This corresponds to a transition from distorted $\mathrm{O}_{\mathrm{h}}$ symmetry (1) to tetragonal $\mathrm{D} 4_{\mathrm{h}}$ symmetry (4) (see the structural models in Figure 8). To understand this it is instructive to analyze how the atomic ${ }^{4} \mathrm{~F}$ multiplet energies split with the so-determined crystal-field parameters $10 \mathrm{Dq}$ and Ds. The first transition $\left({ }^{4} \mathrm{~A}_{2 \mathrm{~g}} \rightarrow{ }^{4} \mathrm{E}_{\mathrm{g}}\right)$ increases in energy from 0.1 to $0.3 \mathrm{eV}$ as decreasing $10 \mathrm{Dq}$ from 0.9 to $0.6 \mathrm{eV}$ and increasing Ds from 0.05 to $0.15 \mathrm{eV}$. While for sample 1 the corresponding peak in the $2 \mathrm{p} 3 \mathrm{~d}$ RIXS spectrum cannot be distinguished from elastic scattering at $0 \mathrm{eV}$ it is clearly visible for samples 2-4. The second transition $\left({ }^{4} \mathrm{~A}_{2 \mathrm{~g}} \rightarrow{ }^{4} \mathrm{~B}_{2 \mathrm{~g}}+{ }^{4} \mathrm{E}_{\mathrm{g}}\right)$ decreases in energy from 0.9 to $0.7 \mathrm{eV}$ for the same $10 \mathrm{Dq}$ and $\mathrm{Ds}$ variation and the corresponding peak in the spectrum gradually merges with the first when going from sample 1 to $\mathbf{4}$. Both effects, the blue shift of the first and the red shift of the second peak, explain the observed changes in the Co $2 p 3 d$ RIXS spectrum and illustrate how ligand coordination and symmetry can be probed in metal complexes with high-resolution 2 p3d RIXS.

The ability to probe spin-forbidden LF excitations (Figure 7) and symmetry effects in metal complexes with $2 \mathrm{p} 3 \mathrm{~d}$ RIXS (Figure 8 ) is combined and exploited in the high-resolution 2p3d RIXS investigation of solid Fe" and Fe"l' compounds, further adding the aspect of varying the metal oxidation state, by Hahn et al. in Figure 9 (Hahn et al., 2017). 
a
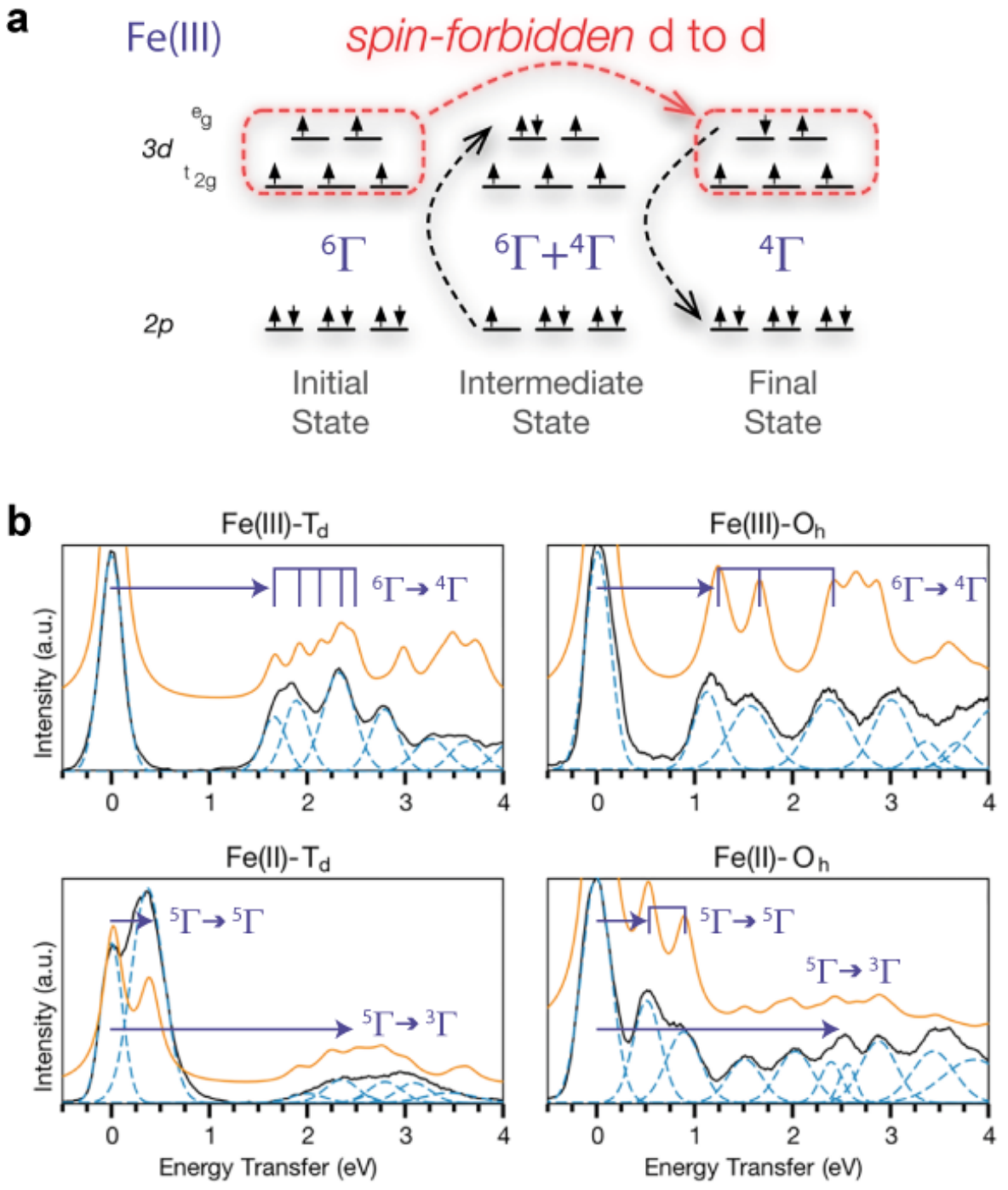

Figure 9. a Schematic molecular-orbital energy diagram of a $\mathrm{d}^{5}\left(\mathrm{Fe}^{\mathrm{III}}\right)$ metal complex in $\mathrm{O}_{\mathrm{h}}$ symmetry illustrating the RIXS process (dashed arrows are $2 p-3 d$ absorption and $3 d-2 p$ emission) for a spin-flip LF(ligand-field, LF) excitation. $\mathbf{b}$ Fe $\mathrm{L}_{3}-\mathrm{RIXS}$ spectra of $\mathrm{Fe}^{\prime \prime}$ and $\mathrm{Fe}^{\mathrm{III}}$ in $\mathrm{Td}$ and $\mathrm{Oh}$ symmetries for $\mathrm{Fe} \mathrm{III}^{\mathrm{C}} \mathrm{Cl}_{4^{-}}$ $\mathrm{Td}$, $\mathrm{Fe}^{\prime \prime \prime} \mathrm{Cl}_{6}-\mathrm{Oh}, \mathrm{Fe}^{\prime \prime} \mathrm{Cl}_{4}-\mathrm{Td}$, and $\mathrm{Fe}^{\prime \prime} \mathrm{Cl}_{6}-\mathrm{Oh}$ (experimental bandwidth $0.3 \mathrm{eV}$, black: measured spectra, blue: fitted peaks, orange: crystal-field multiplet CFM calculations). Adapted with permission from (Hahn et al., 2017). Copyright 2017 American Chemical Society.

The schematic representation of Fe 2p3d RIXS in Figure 9a from the initial ground state through the intermediate core-excited to the final valence-excited states illustrates the concept of probing nominally spin-forbidden LF excitations in $\mathrm{Fe}^{\prime \prime \prime}$ complexes in $\mathrm{O}_{h}$ symmetry. In such a high-spin configuration with sextet ground state $\left({ }^{6} \mathrm{~A}_{1 \mathrm{~g}}\right.$, ${ }^{6} \Gamma$ in Figure $\left.9 a\right)$ all metal-dominated $e_{g}$ and $t_{2 g}$ orbitals are singly occupied and therefore all LF excitations are strictly spin (and parity) forbidden. In fact, LF excitations are spin-forbidden for all metal complexes with high-spin $d^{5}$ ground state. In Fe L-edge RIXS, in contrast, the strong spinorbit interaction in the intermediate core-excited states enables transitions to spin-flip final 
states, where the metal $3 \mathrm{~d}$ spin orientations differ in the initial and final states, also in pure $\mathrm{O}_{\mathrm{h}}$ symmetry (Figure 9a). The underlying reason is that the Fe $2 \mathrm{p}$ spin-orbit interaction (order of magnitude $8 \mathrm{eV}$, given by the Fe $2 p$ spin-orbit parameter $\zeta(2 p)$ (van der Laan and Kirkman, 1992)) is much stronger than the $3 \mathrm{~d}$ spin orbit interactions (order of magnitude 0.1 $\mathrm{eV}$, given by the Fe 3d spin-orbit parameter $\zeta(3 \mathrm{~d})$ (van der Laan and Kirkman, 1992)). For 2p3d RIXS of Fe $\mathrm{F}^{\prime \prime \prime}-\mathrm{O}_{h}$ the Fe $2 \mathrm{p}^{5}\left(\mathrm{e}_{\mathrm{g}}, \mathrm{t}_{2 \mathrm{~g}}\right)^{6}$ intermediate core-excited states can be described as a mixture of sextet and quartet states $\left({ }^{6} \Gamma+{ }^{4} \Gamma\right.$ in Figure 9a) resulting in considerable transition probabilities from the sextet ground state to quartet final states ( ${ }^{4} \Gamma$ in Figure $9 a$ ). ${ }^{6} \Gamma \rightarrow{ }^{4} \Gamma$ transitions therefore give rise to the corresponding peaks in the RIXS spectrum and they appear at energy transfers between 1 and $3 \mathrm{eV}$ as displayed in Figure $9 \mathrm{~b}$ (note that ${ }^{6} \Gamma \rightarrow{ }^{6} \Gamma$ transitions correspond to elastic scattering at $0 \mathrm{eV}$ and no other spin-preserving LF transitions are possible in the high-spin $d^{5}$ configuration of $\mathrm{Fe}^{\mathrm{III}}-\mathrm{O}_{\mathrm{h}}$ ). These RIXS peaks are well described by the CFM model shown as orange line in Figure 9b with a 10Dq value of 1.5 eV. Similar to the preceding case of Co $2 p 3 d$ RIXS in Figure 8, the calculated CFM spectra were obtained by finding the best match between measured and calculated spectra for the thereby determined input values of the CFM model $(10 \mathrm{Dq}$ and the atomic integrals quantifying $3 d-3 d$ Coulomb interactions, namely the Coulomb integrals, also denoted SlaterCondon parameters, $F^{2}(3 d, 3 d)$ and $F^{4}(3 d, 3 d)$ where the latter are uniquely related to the

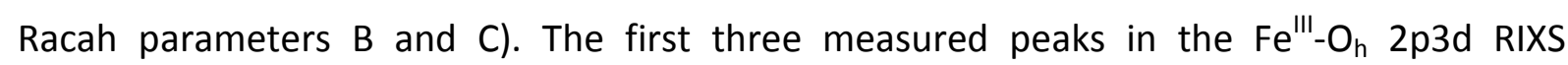
spectrum at $1.1,1.6$, and $2.4 \mathrm{eV}$ in Figure $9 \mathrm{~b}$ can in this way be assigned to the nominally spin-forbidden transitions from the ${ }^{6} \mathrm{~A}_{1 \mathrm{~g}}$ ground state to ${ }^{4} \mathrm{~T}_{1 \mathrm{~g}},{ }^{4} \mathrm{~T}_{4 \mathrm{~g}}$, and ${ }^{4} \mathrm{~A}_{1 \mathrm{~g}}$ final states, respectively.

To further demonstrate the information content of $2 p 3 d$ RIXS we consider how the Fe $2 p 3 d$ RIXS spectrum of Fe $\mathrm{F}^{\text {III }}$ changes with local site symmetry when going from $\mathrm{O}_{h}$ to $T_{d}$ symmetry (Figure 9b). It is important to repeat here that the sensitivity of $2 \mathrm{p} 3 \mathrm{~d}$ RIXS to the symmetry locally at the metal site is given the localized $\mathrm{Fe}_{3}$ absorption edge and that probing the local site symmetry with ligand-field spectra is uniquely enabled with 2p3d RIXS by direct access to the spin-forbidden LF excitations. The measured Fe ${ }^{I I I}-T_{d}$ RIXS spectrum starts at higher energy transfers compared to the $\mathrm{Fe}^{\mathrm{III}}-\mathrm{O}_{\mathrm{h}}$ spectrum with a first peak at $1.7 \mathrm{eV}\left({ }^{6} \mathrm{~A}_{1 \mathrm{~g}} \rightarrow{ }^{4} \mathrm{~T}_{1}\right)$ in $\mathrm{Fe}^{\mathrm{III}-}-\mathrm{T}_{\mathrm{d}}$ compared to $1.1 \mathrm{eV}\left({ }^{6} \mathrm{~A}_{1 \mathrm{~g}} \rightarrow{ }^{4} \mathrm{~T}_{1 \mathrm{~g}}\right)$ in $\mathrm{Fe}^{\mathrm{III}}-\mathrm{O}_{\mathrm{h}}$. This directly reflects the increase of the

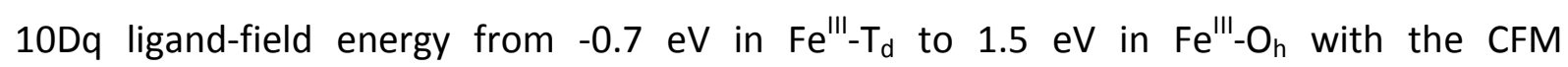
calculations. 
The comparison of $\mathrm{Fe}^{\mathrm{III}}-\mathrm{T}_{\mathrm{d}} / \mathrm{Fe}^{\mathrm{III}}-\mathrm{O}_{\mathrm{h}}$ to $\mathrm{Fe}^{\prime \prime}-\mathrm{T}_{\mathrm{d}} / \mathrm{Fe}^{\prime \prime}-\mathrm{O}_{\mathrm{h}}$ in Figure $9 \mathrm{~b}$ then shows how the Fe $2 \mathrm{p} 3 \mathrm{~d}$ RIXS spectrum reflects changes in the variation of the metal oxidation state and ligand coordination. Most notably, and in contrast to their ferric counterparts discussed in the first section of this chapter, the ferrous $\mathrm{Fe}^{\| l} 2 \mathrm{p} 3 \mathrm{~d}$ RIXS spectra for both $\mathrm{T}_{d}$ and $\mathrm{O}_{h}$ symmetry exhibit strong LF transitions at comparably low energies of $0.3 \mathrm{eV}$ in $\mathrm{Fe}^{\mathrm{Il}}-\mathrm{T}_{\mathrm{d}}$ and $0.5 \mathrm{eV}$ for $\mathrm{Fe}^{\mathrm{II}}-\mathrm{O}_{\mathrm{h}}$ (Figure 9b). These are due to the spin-allowed ${ }^{5} \Gamma \rightarrow^{5} \Gamma$ transitions between the e/ $\mathrm{t}_{2 \mathrm{~g}}$ and $t_{2} / e_{g}$ orbitals in $T_{d} / O_{h}$ symmetry. This is also where the information from Fe $2 p 3 d$ RIXS can now be compared in detail with results from optical measurements by UV/Vis absorption and magnetic circular dichroism measurements and we refer to the original paper by Hahn et al. for this discussion (Hahn et al., 2017). As for Fe"', the Fe" RIXs spectra in Figure $9 \mathrm{~b}$ also show spin-forbidden transitions $\left({ }^{5} \Gamma \rightarrow^{3} \Gamma\right.$ for Fe" $)$ at energy transfers of above $1 \mathrm{eV}$.

Two seemingly subtle details in Figure $9 \mathrm{~b}$ finally demonstrate the limitations and point to future improvements of current experimental realizations and their theoretical interpretations of high-resolution 2 p3d RIXS of transition-metal complexes. First, the calculated CFM spectrum of $\mathrm{Fe}^{\|}-\mathrm{T}_{d}$ deviates significantly from experiment for the first inelastic peak at around $0.3 \mathrm{eV}$ that is due to ${ }^{5} \Gamma \rightarrow{ }^{5} \Gamma$ transitions. The apparently missing intensity in the calculation could be due to the restriction to pure tetrahedral symmetry in the calculated spectrum with only one allowed $\left({ }^{5} \mathrm{E} \rightarrow{ }^{5} \mathrm{~T}\right)$ transition. Deviations from pure tetrahedral symmetry in experiment by the Jahn-Teller effect are thought to split this transition giving rise to a multiplet of excitations. Higher resolution in future $2 \mathrm{p} 3 \mathrm{~d}$ RIXS measurements with a bandwidth of less than the $0.3 \mathrm{eV}$ used here would be desirable to better distinguish these features from the elastic scattering at $0 \mathrm{eV}$, to get access to the fine structure and to better match the resolution of optical measurements. Second, the simulated Fe" ${ }^{\text {III }} \mathrm{T}_{d}$ spectra in Figure 9b appear to be "too sharp" compared to experiment (see in particular the energy region around $2 \mathrm{eV}$ ). This again could be due to the limitation of the calculations to pure tetrahedral symmetry and suggests including structures deviating from this idealized symmetry to account for inhomogeneous broadening of the spectra. These observations call for taking into account in the calculated 2p3d RIXS spectra the splitting and broadening of transitions caused by dynamical effects in the ground- and valence-excited states in the samples at room temperature. This seems even more important in the investigation of metal complexes in solution where only a few experiments have been 
carried out to date. One way to account for inhomogeneous broadening effects would be to use a ground-state molecular dynamics simulation to generate a large set of molecular structures and to sum the calculated $2 \mathrm{p} 3 \mathrm{~d}$ RIXS spectra of the different configurations to simulate related multiplet splittings (Penfold et al., 2014).

These notions are supported by the recent investigation of symmetry distortions in solid Co" complexes with Co 2p3d by Liu et al. shown in Figure 10 (Liu et al., 2016).

a
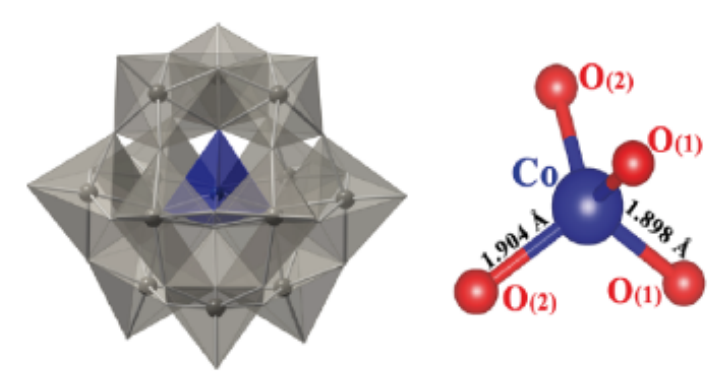

b
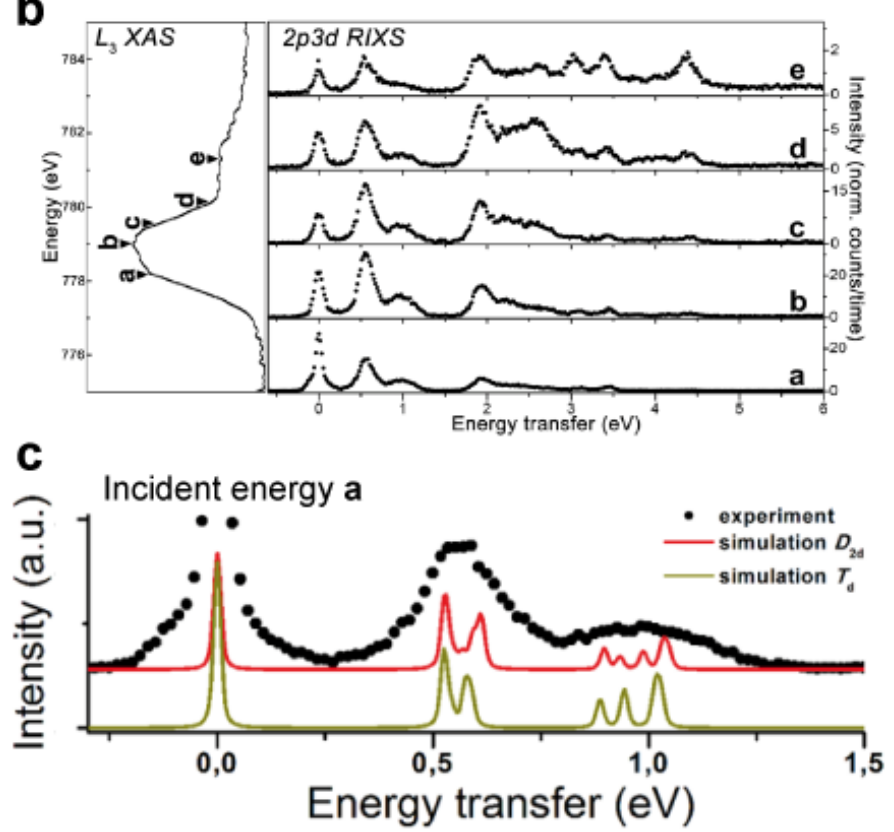

Figure 10. a Left: Structure of the Co polyoxometalate anion $\left[\mathrm{CoW}_{12} \mathrm{O}_{40}\right]^{6-}$ in polyhedral representation (blue: $\mathrm{Co}$ center with oxo ligands, gray: $\mathrm{W}_{12} \mathrm{O}_{40}$ cage), right: $\mathrm{Co}^{\prime \prime}$-oxo core with crystallographic bond lengths. b Co $\mathrm{L}_{3}$-RIXS spectra (experimental bandwidth $0.09 \mathrm{eV}$ ) and $\mathrm{Co}_{3} \mathrm{~L}_{3} \mathrm{x}$-ray absorption spectrum (XAS, experimental bandwidth $0.5 \mathrm{eV}$ ) of $\mathrm{Co}^{11}$ in $\left[\mathrm{CoW}_{12} \mathrm{O}_{40}\right]^{6-}$ (the RIXS spectra a-e were taken at incident photon energies a-e as indicated in the XAS spectrum). $c$ Zoom into the Co ${ }^{\prime \prime} L_{3}$-RIXS spectrum taken at $778 \mathrm{eV}$ (incident photon energy a) as measured (circles) and calculated within the crystal-field multiplet CFM model for Co" in $T_{d}$ (green) and $D_{2 d}$ (red) symmetries (simulated bandwidth smaller than in experiment to highlight the splitting of the peak at $0.6 \mathrm{eV}$ energy transfer). Adapted with permission from (Liu et al., 2016). Copyright 2016 American Chemical Society.

$\mathrm{CO}^{\prime \prime}$ in the studied $\left[\mathrm{CoW}_{12} \mathrm{O}_{40}\right]^{6-}$ anions with a nominal tetrahedral Co"-oxo core (Figure 10a) were studied with $2 p 3 d$ RIXS at the Co $L_{3}$ absorption edge. The observed peaks in the Co 
2p3d RIXS spectrum between 0 and $4 \mathrm{eV}$ (Figure 10b) are dominated by LF excitations with minor contributions from charge-transfer excitations. The study then concentrates on the first two RIXS peaks at 0.5-0.6 and $1 \mathrm{eV}$ (Figure 10c) because they give information on possible distortions from perfect tetrahedral symmetry. These peaks are assigned to spinallowed ${ }^{4} \Gamma \rightarrow{ }^{4} \Gamma$ transitions in $\mathrm{Co}^{11}-\mathrm{T}_{\mathrm{d}}\left({ }^{4} \mathrm{~A}_{2} \rightarrow{ }^{4} \mathrm{~T}_{2}\right.$ at $0.5-0.6 \mathrm{eV}$ and ${ }^{4} \mathrm{~A}_{2} \rightarrow{ }^{4} \mathrm{~T}_{1}$ at $\left.1 \mathrm{eV}\right)$. The calculated CFM spectra of $C 0^{\prime \prime}$ in perfect tetrahedral $\left(T_{d}\right)$ and in tetragonal $\left(D_{2 d}\right)$ symmetry are compared to experiment in Figure 10c (note that the too small broadening applied to the calculated spectra was deliberately chosen such as to emphasize peak shifts instead of aiming at best matching the experiment). The calculated $\mathrm{Co}$ "- $\mathrm{T}_{d}$ spectrum clearly shows the splitting of both the ${ }^{4} \mathrm{~T}_{2}$ and ${ }^{4} \mathrm{~T}_{1}$ peaks due to $3 \mathrm{~d}$ spin-orbit interactions. In the distorted tetrahedral $D_{2 d}$ symmetry with two sets of different Co-O bonds lengths (roughly 1.89 and 1.90-1.97 $\AA$ ) the ${ }^{4} \mathrm{~A}_{2} \rightarrow{ }^{4} \mathrm{~T}_{2}$ and ${ }^{4} \mathrm{~A}_{2} \rightarrow{ }^{4} \mathrm{~T}_{1}$ are found to further split. This is reflected in the calculated $\mathrm{Co}^{\prime \prime}-\mathrm{D}_{2 \mathrm{~d}}$ spectrum compared to the Co"- $\mathrm{T}_{d}$ spectrum in Figure $10 \mathrm{c}$ by the increased multiplet splitting of the RIXS peaks at 0.5-0.6 and $1 \mathrm{eV}$. This could account for part of the measured width of these peaks of around $0.2 \mathrm{eV}$ which is significantly larger than the experimental bandwidth of this study of $0.09 \mathrm{eV}$ and suggests that the studied nominal tetrahedral Co"-oxo cores are tetragonally distorted. The deficiency of the Co"- $\mathrm{D}_{2 \mathrm{~d}}$ calculation to fully account for the measured width, however, also indicates in agreement with the conclusions from the preceding study of $\mathrm{Fe}^{\mathrm{II}}$ and $\mathrm{Fe}^{\mathrm{III}}$ by Hahn et al. that fundamental mechanisms leading to splitting and broadening in the CFM approach are missing. Further, the Co 2p3d RIXS study in Figure 10 shows that RIXS measurements with an even higher resolution, with a bandwidth of well below the reported $90 \mathrm{meV}$ are in fact needed to gain insight into the subtle multiplet splitting of well below $100 \mathrm{meV}$ associated with symmetry distortions in 2p3d RIXS peaks. In the exemplary case of Co" in Figure 10c this splitting is on the order of 20-30 meV for the RIXS peak at 0.5-0.6 eV $\left({ }^{4} \mathrm{~A}_{2} \rightarrow{ }^{4} \mathrm{~T}_{2}\right)$.

The purpose of the last example in this section is to demonstrate the versatility of $2 \mathrm{p} 3 \mathrm{~d}$ RIXS in terms of accessing different classes of materials. While the preceding discussion focused on mononuclear metal complexes and zoomed in on more and more detailed insight into ligand coordination and symmetry, the example in Figure 11 by Cui et al. addresses metal 2 p3d RIXS as a probe of nanoparticle size and composition and how these relate to the probed electronic structure (Cui et al., 2017). 

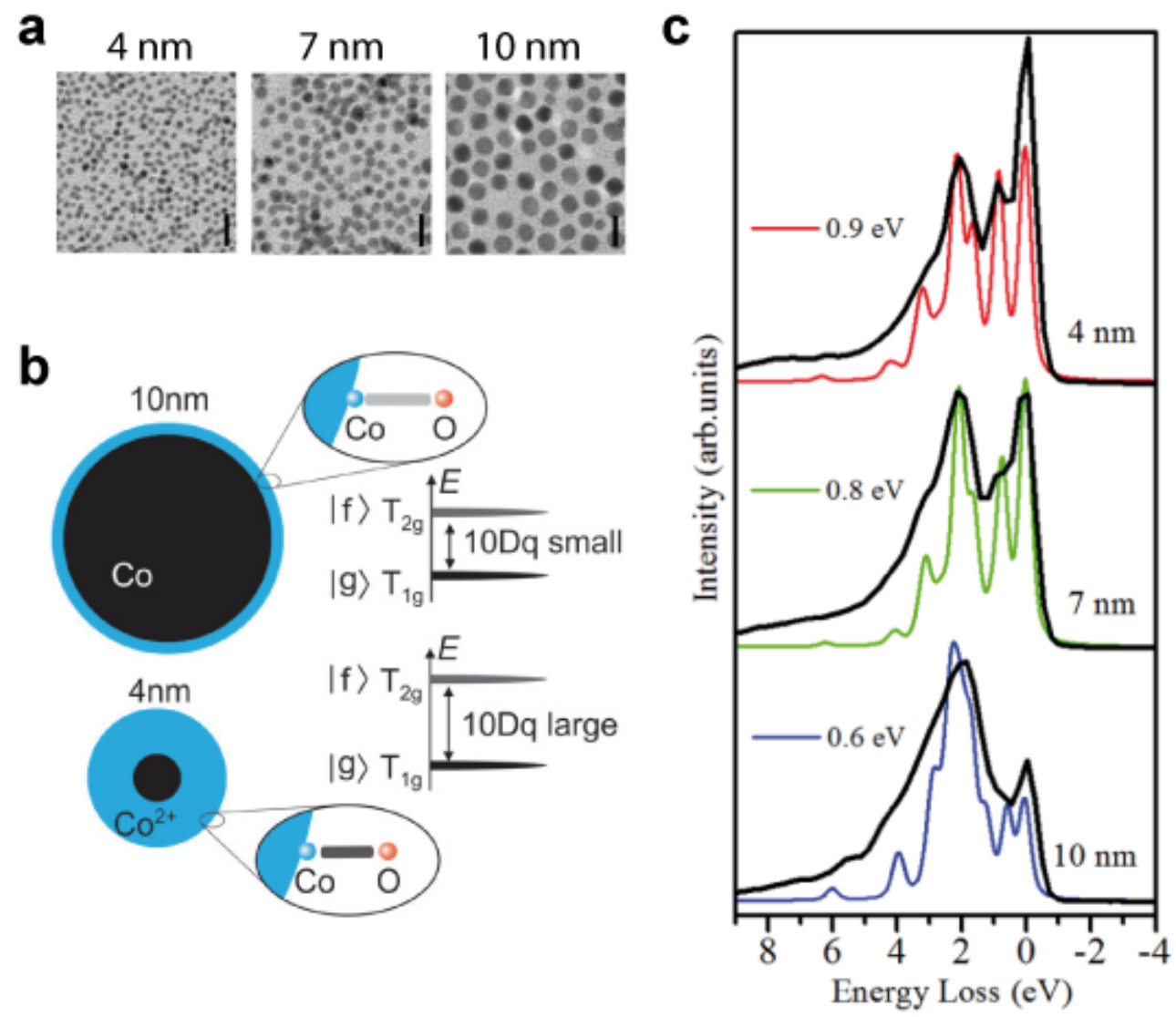

Figure 11. a TEM images of monodispersed partially oxidized Co nanoparticles (diameters 4, 7 and 10 $\mathrm{nm}$, scale bars equal $20 \mathrm{~nm}$ ). b Conceptual depiction of the relationship between Co nanoparticle size, Co-oxide contribution and ligand field 10Dq (black: metallic Co, blue: Co" oxide with a small contribution in $10 \mathrm{~nm}$ particles and a large contribution in $4 \mathrm{~nm}$ particles, Co-O bonds in the nanoparticle surface-oxide phase are schematically shown). c Co $\mathrm{L}_{3}$-RIXS spectra as measured for Co nanoparticles of 4, 7 and $10 \mathrm{~nm}$ sizes (black, experimental bandwidth $0.5 \mathrm{eV}$ ) and as calculated within the crystal-field multiplet CFM model $\left(\mathrm{Co}^{\prime \prime}\right.$ in $\mathrm{O}_{\mathrm{h}}$ symmetry with ligand-field parameters 10Dq of 0.9 eV: red, $0.8 \mathrm{eV}$ : green, and $0.6 \mathrm{eV}$ : blue). Adapted with permission from (Cui et al., 2017). Copyright 2017 American Chemical Society.

Cui et al. studied partially oxidized Co nanoparticles of different sizes (with diameters of 4, 7 and $10 \mathrm{~nm}$, TEM images are shown in Figure 11a). The idea of the investigation, illustrated in Figure 10b, is conceptually simple but very powerful: Co nanoparticles of different sizes contain different amounts of surface-oxidized Co-oxide phases where the oxide in the larger/smaller particles is thinner/thicker with respect to the particle size (with respect to the metallic Co core). Now the authors reason that the resilience to oxidation correlates with the Co-O bond strength in the oxide phase were weakly/strongly oxidized nanoparticles exhibit weak/strong Co-O bonds. This variation in bond strength correlates with a variation of the ligand-field energy 10Dq and should thus be reflected in the LF excitation energies in the Co $2 p 3 d$ RIXS spectra of the nanoparticles. And indeed, the measured Co $2 p 3 d$ RIXS 
spectra in Figure 10c show systematic variations with nanoparticle size in terms of increasing intensity at energy transfers of $2 \mathrm{eV}$ and decreasing intensity at around $1 \mathrm{eV}$ with increasing particle size. It is important to note that Cui et al. used here the ability of $2 p 3 d$ RIXS to selectively enhance features of certain species or to select certain chemical environments of the probed element via their distinctive $x$-ray resonance energies: The spectra in Figure 10c were taken at an incident photon energy corresponding to a peak in the Co L-edge XAS spectrum that is known to arise from $\mathrm{CoO}$ (in contrast to the underlying absorption spectrum of metallic Co where this peak is missing).

With the preceding examples in this section the spectral changes in the $2 p 3 d$ RIXS spectra in Figure 11c can now be understood in terms of how the LF excitations vary with electronic structure in the $\mathrm{CoO}$ phases of the nanoparticles (CT excitations are found to arise above 7 $\mathrm{eV}$ in the spectra and are not addressed here). In $\mathrm{CoO}$ the $\mathrm{Co}^{\prime \prime}$ ions are in an $\mathrm{O}_{\mathrm{h}}$ ligand field and the Co L-RIXS peaks at 1 and $2 \mathrm{eV}$ are due to spin-allowed ${ }^{4} \Gamma \rightarrow{ }^{4} \Gamma$ transitions from the $\mathrm{Co}^{11}-\mathrm{O}_{\mathrm{h}}{ }^{4} \mathrm{~T}_{1 \mathrm{~g}}$ ground state to ${ }^{4} \mathrm{~T}_{2 \mathrm{~g}}$ and ${ }^{4} \mathrm{~T}_{1 \mathrm{~g}}+{ }^{4} \mathrm{~A}_{2 \mathrm{~g}}$ states, respectively. The energy of the ${ }^{4} \mathrm{~T}_{1 \mathrm{~g}} \rightarrow{ }^{4} \mathrm{~T}_{2 \mathrm{~g}}$ transition at around $1 \mathrm{eV}$ therefore directly reflects the ligand-field strength and the $10 \mathrm{Dq}$ values estimated from the measured $2 \mathrm{p} 3 \mathrm{~d}$ RIXS spectra are $0.9,0.8$ and $0.6 \mathrm{eV}$ for the nanoparticles with sizes of 4,7 and $10 \mathrm{~nm}$, respectively. This is directly visible in the spectra in Figure $11 \mathrm{c}$ where the peak at around $1 \mathrm{eV}$ is well separated from elastic scattering for the $4 \mathrm{~nm}$ particles while it red-shifts with increasing particle size to merge with the elastic scattering peak at $0 \mathrm{eV}$ for the $10 \mathrm{~nm}$ particles. This is confirmed by the calculated CFM spectra in Figure 11c for $10 \mathrm{Dq}$ values of $0.9,0.8$ and $0.6 \mathrm{eV}$ and nicely confirms the conceptual idea of this investigation (Figure 10b). The calculations also reproduce the experimentally observed increase of intensities of the ${ }^{4} \mathrm{~T}_{1 \mathrm{~g}} \rightarrow{ }^{4} \mathrm{~T}_{1 \mathrm{~g}}+{ }^{4} \mathrm{~A}_{2 \mathrm{~g}}$ transitions around $2 \mathrm{eV}$ for increasing nanoparticle size. This study therefore exemplifies the level of detail to which the electronic structure of selected environments in nanoparticles can be probed, an insight that could prove essential in the investigation and mechanistic understanding of the catalytic performance of such systems.

We want to mention that further interesting studies of LF excitations in metal compounds and catalytic systems were recently reported by the groups of DeBeer (Van Kuiken et al., 2016, Van Kuiken et al., 2018) and deGroot (Hunault et al., 2018, Liu et al., 2017, van Schooneveld et al., 2013b) and others (Asakura et al., 2014, Bokarev et al., 2015, Miedema et al., 2017). 


\section{Metal-ligand covalency from charge-transfer excitations}

This section expands upon the previous one by considering electronic excitations at higher energy transfers beyond the LF transitions in $2 p 3 d$ RIXS spectra of metal complexes and compounds to show how this energy region may give access to the metal-ligand orbital covalency. It is motivated by the driving question: What else can we learn from $2 p 3 d$ RIXS maps?

Investigating CT excitations is the starting point for the idea to extract information on the nature of metal-ligand bonds and their covalency because CT transitions involve ligandcentered orbitals. Making use of the metal L-edge absorption transition to the RIXS intermediate states is another important aspect of the underlying concept because in a simplified way, CT excitations in $2 p 3 d$ RIXS can be viewed as arising from a projection of the delocalized covalent metal-ligand orbitals to the localized metal $2 p$ core hole. At the time of publication of this book chapter, investigations of metal-ligand covalency with $2 p 3 d$ RIXS are less common compared to studies on LF excitations. One reason for this may be the difficulty of theoretically describing metal-ligand covalency and CT excitations in metal complexes accurately. The CTM model (De Groot, 2001, De Groot and Kotani, 2008) addresses this need and extends the CFM model by including CT configurations with holes in ligand orbitals and additional electrons on the metal. It has been used successfully by Solomon and co-workers to extract metal-ligand covalency from metal K- and L-edge XAS spectra (Hocking et al., 2007, Wasinger et al., 2003) as well as metal K-edge RIXS (Kroll et al., 2014, Lundberg et al., 2013). Restricted active space (RAS) and DFT-ROCIS calculations (Josefsson et al., 2012, Preusse et al., 2016, Roemelt et al., 2013, Pinjari et al., 2014) have emerged as an alternative way of describing charge transfer in $\mathrm{K}$ - and L-edge spectra of metal complexes.

Here we discuss recent $2 p 3 d$ RIXS investigations that aimed at transferring the concepts for extracting metal-ligand covalency from CT excitations in RIXS at the metal $\mathrm{K}$ - to the metal Ledges. We note that these are investigations of comparably ionic systems with weak ligand fields (small metal-ligand orbital overlap) where LF and CT RIXS peaks can be well separated. In the examples discussed here the LF excitations are in the range of 0-5 eV followed by CT excitations extending from around 5 to $15 \mathrm{eV}$. Whether or how the presented concepts can be transferred to other and in particular to more covalent systems will have to be addressed 
in future studies. In addition it will be interesting to see whether the concept is applicable to cases where LF and CT excitations overlap or where, such as in the iron hexacyanides (Figure 3), LF excitations can be separated from CT excitations by proper choice of the incident energy.

We start by discussing the simple case of Ni $2 p 3 d$ RIXS of the octahedral aqueous complex $\left[\mathrm{Ni}\left(\mathrm{H}_{2} \mathrm{O}\right)_{6}\right]^{2+}$ again by now extending with Figure 12 the discussion of LF excitations from the previous section to the investigation of CT excitations (Kunnus et al., 2017).

a

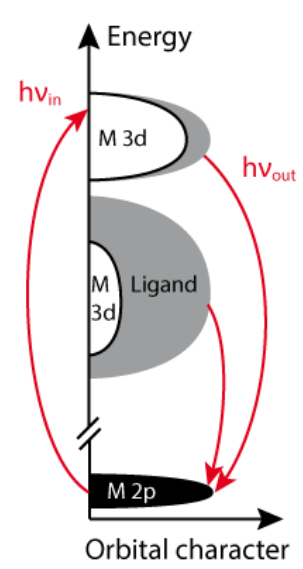

b

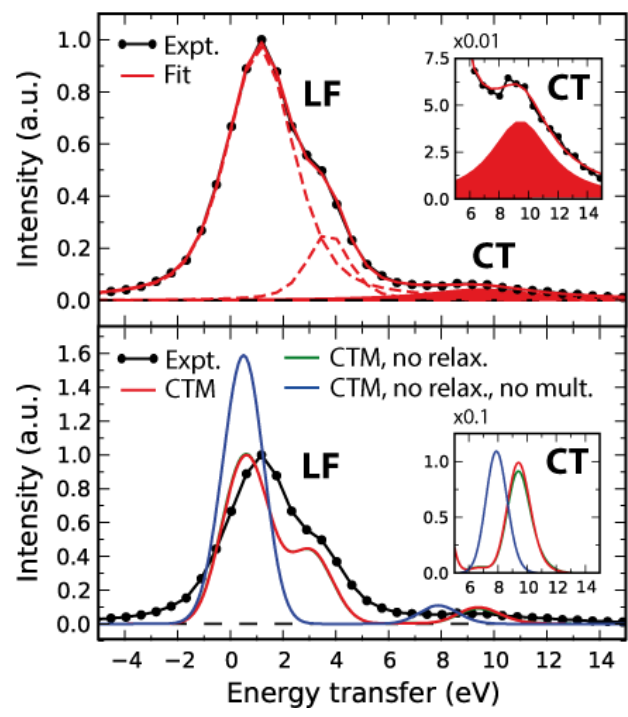

Figure 12. a Schematic depiction of orbital character versus orbital energies of metal (M) L-edge RIXS (2p-3d absorption at $h v_{i n}, 3 d-2 p$ emission at $h v_{\text {out }}$ for two possible transitions from two sets of valence orbitals) with metal $2 p$ orbitals (black) and metal $3 d$ and ligand contributions in white and gray, respectively, for the two sets of valence orbitals. Corresponding schematic metal L-edge RIXS spectrum with a ligand-field (LF) peak and a charge-transfer (CT) peak (final-state configurations are given for the example of $\mathrm{Ni}^{\prime \prime}$ L-edge RIXS, see text). $\mathbf{b}$ Ni $\mathrm{L}_{3}$-RIXS spectra of $\mathrm{Ni}^{\prime \prime}$ in $\mathrm{O}_{\mathrm{h}}$ symmetry with LF excitations at $0-4 \mathrm{eV}$ and $\mathrm{CT}$ excitations around $10 \mathrm{eV}\left(\mathrm{Ni}^{\mathrm{il}} \text { in }\left[\mathrm{Ni}^{(} \mathrm{H}_{2} \mathrm{O}\right)_{6}\right]^{2+}$ from $\mathrm{NiCl}_{2}$ in aqueous solution, black: experiment, bandwidth $1 \mathrm{eV}$, top red: fit, bottom red/green/blue: crystal-field multiplet CTM calculations, see text). Reprinted from (Kunnus et al., 2017), with permission from Elsevier.

Due to the weak covalent interactions between $\mathrm{Ni}^{\mathrm{Il}}$ and water solvent molecules the CT interactions can be expected to be weak but this simple case is still ideally suited to introduce the concept. The idea of using CT excitations in 2p3d RIXS to probe covalency by projecting the metal-ligand orbitals to the localized metal $2 p$ core hole is illustrated in Figure 12a. The two sets of schematically depicted orbitals differ in their orbital character and constitute different degrees of metal and ligand contributions with metal-dominated orbitals at higher energies (large portion of white "M 3d" in Figure 12a, these are partially occupied) 
and ligand-dominated orbitals at lower energies (large portion of gray "Ligand" in Figure 12a, these are occupied, unoccupied orbitals are omitted in Figure 12a).

Metal 2p3d RIXS, within the conceptual one-electron picture presented in Figure 12, is shown for the $2 p$ to unoccupied $3 d$ excitation ( $h v_{\text {in }}$ in Figure $\left.12 a\right)$ followed by one-electron transitions back to the $2 p$ core hole either from the metal- or from the ligand-dominated set of orbitals ( $h v_{\text {out }}$ in Figure 12a). This second step corresponds to projecting, via the dipole operator for radiative transitions, the valence orbitals onto the localized metal $2 p$ core hole and this is where the idea for extracting covalency emerges. The $d \rightarrow p$ dipole-transitions back to the $2 p$ core hole are stronger for larger metal $3 d$ contributions to the involved valence orbitals and for stronger mixing of metal-centered and ligand-centered configurations in the $2 p$ core-hole state (Hocking et. al., 2007). The covalency of the metalligand bond can thus be expected to be reflected in the intensity of CT transitions with respect to the LF transitions: strong covalency corresponds to a large degree of metal $3 \mathrm{~d}$ character of the ligand-dominated orbitals and is reflected in strong CT excitations.

With the ground-state valence-electron configuration $\left(\mathrm{Le}_{\mathrm{g}}, \mathrm{Lt}_{2 \mathrm{~g}}\right)^{10}\left(\mathrm{t}_{2 \mathrm{~g}}\right)^{6}\left(\mathrm{eg}_{\mathrm{g}}\right)^{2}$ of $\left[\mathrm{Ni}\left(\mathrm{H}_{2} \mathrm{O}\right)_{6}\right]^{2+}$ it is now clear that for $\left[\mathrm{Ni}\left(\mathrm{H}_{2} \mathrm{O}\right)_{6}\right]^{2+}$ the metal-dominated set of orbitals in Figure 12a can be identified with the Ni-dominated $3 d$ antibonding $t_{2 g}$ and $e_{g}$ orbitals (where the higher-lying $\mathrm{e}_{\mathrm{g}}$ are partially unoccupied) while the ligand-dominated set of orbitals in Figure 12a are identified with water-dominated bonding $L_{g}$ and $L_{2 g}$ orbitals. These orbitals result from covalent orbital mixing of $\mathrm{Ni} 3 d$ and water $3 a_{1}$ and $1 b_{1}$ orbitals and of the resulting antibonding and bonding combinations only the relevant orbitals are discussed here with Figure 12 (other occupied orbitals of $t_{2 u}, t_{1 u}$ and $a_{1 g}$ symmetry in particular are omitted here for clarity). Covalency of the Ni-water bond can therefore be quantified by the amount of $\mathrm{Ni}$ $3 d$ character in the ligand-centered bonding $L e_{g}$ and $L_{2 g}$ orbitals or, equivalently, by the amount of ligand character in the $\mathrm{Ni}$-centered antibonding $\mathrm{e}_{\mathrm{g}}$ and $\mathrm{t}_{2 \mathrm{~g}}$ orbitals and the latter will be done here.

Ni 2p3d RIXS then results in the LF final states discussed in the previous section (Figure 7) via $L F\left(t_{2 g} \rightarrow e_{g}\right)$ transitions or in the CT final states via the $\left(L e_{g}, L t_{2 g}\right) \rightarrow\left(e_{g}, t_{2 g}\right) C T$ transitions (Figure 12a). According to the expected correlation between metal-ligand covalency, orbital mixing or character and intensity of LF and CT peaks, the LF $\left(\mathrm{e}_{\mathrm{g}}, \mathrm{t}_{2 \mathrm{~g}}\right)^{8}$ peaks in the weakly covalent complex $\left[\mathrm{Ni}\left(\mathrm{H}_{2} \mathrm{O}\right)_{6}\right]^{2+}$ should be stronger than the integrated CT $\left(\mathrm{Le}_{\mathrm{g}}, \mathrm{Lt}_{2 \mathrm{~g}}\right)^{9}\left(\mathrm{e}_{\mathrm{g}}, \mathrm{t}_{2 \mathrm{~g}}\right)^{9}$ peaks as 
illustrated in Figure 12a. The measured Ni 2p3d RIXS spectrum of $\left[\mathrm{Ni}\left(\mathrm{H}_{2} \mathrm{O}\right)_{6}\right]^{2+}$ in Figure $11 \mathrm{~b}$ confirms this expectation: The LF intensities at $0-5 \mathrm{eV}$ are much stronger than the CT transitions at around 7-11 eV. In addition, the relative intensities of CT with respect to LF peaks can be quantified (see the fitted Gaussians in Figure 12b for quantification of integrated LF and CT intensities). This CT/LF intensity ratio then relates to the amount of ligand-orbital character in the $\mathrm{Ni}$-centered orbitals, i.e. the $\mathrm{Ni}$-water bond covalency. This approach results in a CT/LF intensity ratio of 0.065 for the spectrum in Figure $12 \mathrm{~b}$ and a corresponding amount of $5.5 \%$ of water-ligand orbital character in the $\mathrm{Ni}$-centered $\mathrm{e}_{\mathrm{g}} / \mathrm{t}_{2 \mathrm{~g}}$ orbitals reflecting the comparably weak covalency of the $\mathrm{Ni}$-water bond in the $\left[\mathrm{Ni}\left(\mathrm{H}_{2} \mathrm{O}\right)_{6}\right]^{2+}$ complex.

$\mathrm{Ni} 2 \mathrm{p} 3 \mathrm{~d}$ RIXS spectra as calculated within the CTM model for $\mathrm{Ni}^{\prime \prime}-\mathrm{O}_{\mathrm{h}}(10 \mathrm{Dq}=1.1 \mathrm{eV})$ are compared to experiment in Figure 12b. Here the CTM model includes the nominal ionic $\mathrm{Ni}^{\prime \prime}$ $3 d^{8}$ configuration and $3 d^{9} L^{-1}$ where $L^{-1}$ denotes a hole in the ligand orbitals to account for metal-ligand covalency (note that this describes the covalency in the electronic ground state of the system). This way of describing covalency by a configuration corresponding to ligand to metal charge transfer is inherent to the CTM model and charge transfer is quantified by the so-called charge-transfer energy $\Delta$ (with a value of $6.5 \mathrm{eV}$ in the case here) (De Groot, 2001, De Groot and Kotani, 2008). The charge-transfer energy $\Delta$ is the average energy between the multiplets due to the nominal $3 d^{n}$ configuration and the charge-transfer configuration $3 d^{n+1} L^{-1}$. The smaller this energy the stronger is the hybridization of metal and ligand orbitals. A stronger hybridization corresponds to stronger covalency (De Groot, 2001, De Groot and Kotani, 2008). The CTM model then allows transferring these ground-state considerations via the core-excited states to the RIXS final states, thereby accounting for the influence of the core hole to some extent. The CTM calculation in Figure 12b confirms the assignments of LF transitions and CT transitions, and the calculated CT/LF intensity ratio of 0.066 compares well with experiment (0.065) thereby confirming the experimentally determined covalency of the $\mathrm{Ni}$-water bonds in $\left[\mathrm{Ni}\left(\mathrm{H}_{2} \mathrm{O}\right)_{6}\right]^{2+}$. The additional calculations shown Figure 12b correspond to various degrees of approximations indicating that it will be interesting to investigate the influence of multi-electron correlations (multiplet effects), core-hole screening or relaxation effects and RIXS channel interference on the determination of covalency with CT and LF intensities in 2p3d RIXS spectra. It will furthermore be interesting to see with future studies whether or to what extent the so-determined 
covalency compares to modern quantum-chemistry calculations in this complex. Finally, the question arises whether the presented concept is generally valid and whether it can be applied to other and in particular to more covalent complexes. This is addressed with the following discussion of a Co $2 \mathrm{p} 3 \mathrm{~d}$ RIXS investigation of a series of Co" compounds with varying degrees of covalency by Wang et al. in Figure 13 (Wang et al., 2017). 

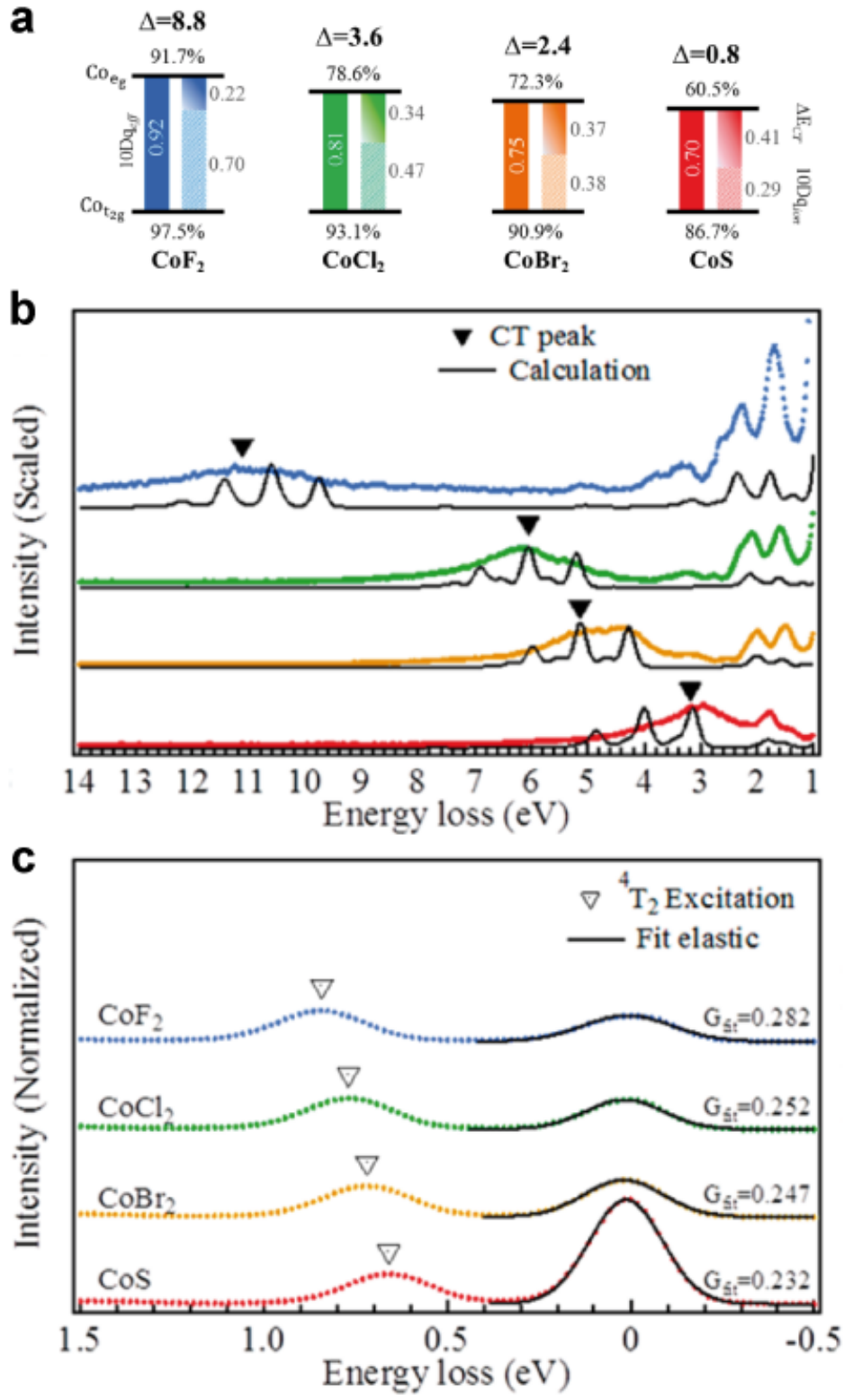

Figure 13. a Schematic molecular-orbital energy diagram illustrating the variation of the electronic structure of $\mathrm{Co}^{\prime \prime}$ in $\mathrm{O}_{\mathrm{h}}$ symmetry from $\mathrm{CoF}_{2}$, to $\mathrm{CoCl}_{2}, \mathrm{CoBr}_{2}$ and $\mathrm{CoS}$ (ligand-field splitting $10 \mathrm{Dq}$ eff in eV decomposed into the ionic ligand-field splitting $10 \mathrm{Dq}_{\text {ion }}$ and the charge-transfer contribution $\Delta \mathrm{E}_{\mathrm{CT}}$, the charge-transfer energy $\Delta$ in $\mathrm{eV}$, and covalencies of $\mathrm{e}_{\mathrm{g}}$ and $\mathrm{t}_{2 \mathrm{~g}}$ orbitals in terms of \% amounts of Co $3 d$ character). b Co $\mathrm{L}_{3}$-RIXS spectra of $\mathrm{Co}^{\prime \prime}-\mathrm{O}_{\mathrm{h}}$ in $\mathrm{CoF}_{2}$, to $\mathrm{CoCl}_{2}, \mathrm{CoBr}_{2}$ and $\mathrm{CoS}$ with ligand-field (LF) excitations up to 2-3 eV and charge-transfer excitations at higher energies ( $\mathrm{CT}$, black triangles, colored circles: experiment, solid black lines: charge-transfer multiplet, CTM, model calculations). c Measured and calculated zooms into the low-energy regions of the RIXS spectra from $\mathbf{b}$ (colored circles: experiment, solid black lines: fits to the data around $0 \mathrm{eV}$ with Gaussian FWHM in eV) with elastic scattering at $0 \mathrm{eV}$ and the first LF excitation (open triangles). Adapted with permission from (Wang et al., 2017). Copyright 2017 American Chemical Society.

The authors measured and calculated with the CTM model the Co 2p3d RIXS spectra of solid $\mathrm{CoF}_{2}, \mathrm{CoCl}_{2}, \mathrm{CoBr}_{2}$ and $\mathrm{CoS}$. Already the ground-state electronegativity values of $\mathrm{F}$ (3.98), $\mathrm{Cl}$ (3.16), $\mathrm{Br}(2.96)$, and $\mathrm{S}$ (2.58) indicate that $\mathrm{CoF}_{2}$ is the most ionic, while $\mathrm{CoS}$ is the least ionic 
compound and therefore the (ground-state) metal-ligand covalency is expected to increase in this series from $\mathrm{CoF}_{2}$ with to CoS. And indeed, the CTM calculations by Wang et al. for Co"$\mathrm{O}_{\mathrm{h}}$ including the nominal $\mathrm{Co}^{11} 3 \mathrm{~d}^{7}$ configuration and the charge-transfer configuration $3 d^{8} \mathrm{~L}^{-1}$ to account for ground-state covalency in these compounds confirms this. The summarized calculated parameters in Figure 13a quantify how the covalency gradually increases from $\mathrm{CoF}_{2}$ to $\mathrm{CoS}$ as indicated by the gradually decreasing charge-transfer energy $\Delta$ (increasing hybridization and covalency) in the CTM calculations from $8.8 \mathrm{eV}$ in $\mathrm{CoF}_{2}$, to $3.6 \mathrm{eV}$ in $\mathrm{CoCl}_{2}$, $2.4 \mathrm{eV}$ in $\mathrm{CoBr}_{2}$ and 0.8 in $\mathrm{CoS}$. The correspondingly decreasing amounts of Co $3 \mathrm{~d}$ character in the $e_{g}$ and $t_{2 g}$ orbitals are also extracted from the calculations and given in Figure 13a. They vary from $92 \%$ for $\mathrm{e}_{\mathrm{g}}$ and $98 \%$ for $\mathrm{t}_{2 \mathrm{~g}}$ in $\mathrm{CoF}_{2}$ to $61 \%\left(\mathrm{e}_{\mathrm{g}}\right)$ and $87 \%\left(\mathrm{t}_{2 \mathrm{~g}}\right)$ in CoS. It is interesting to note that in contrast to the $\mathrm{Ni}$ case above, where the average covalency was considered (averaged over $e_{g}$ and $t_{2 g}$ symmetries), here the authors distinguish the Co content in $e_{g}$ and $t_{2 g}$ orbitals (Figure 13a). This dissection is often termed differential orbital covalency and has been used in L-edge XAS (Wasinger et al., 2003). Wang et al. find that the amount of $\mathrm{Co} 3 \mathrm{~d}$ character decreases faster in $\mathrm{e}_{\mathrm{g}}$ compared to the $t_{2 \mathrm{~g}}$ orbitals with increasing covalency from $\mathrm{CoF}_{2}$ to $\mathrm{CoS}$ (Figure 13a, remember that from the bonding-antibonding orbital pairs formed by metal-ligand orbital interaction we consider here the antibonding part only and that increased covalency for these means increase ligand character and decrease metal $3 d$ character because in a purely ionic system without interactions these antibonding orbitals are pure metal $3 d$ orbitals). This difference between $e_{g}$ and $t_{2 g}$ orbitals in terms of Co $3 \mathrm{~d}$ content can be easily understood by considering the symmetry of the involved orbitals. In octahedral symmetry the Co $3 d$ orbitals of $e_{g}$ symmetry, namely the $3 d$ orbitals of $d\left(x^{2}-y^{2}\right)$ and $d\left(z^{2}\right)$ symmetry, directly point to the ligands along the metal-ligand bond axes. They therefore more strongly overlap with ligand $2 p$ orbitals than the $t_{2 g}$ orbitals of $d(x y), d(x z)$, and $d(y z)$ symmetry, which point to "in-between the ligands". Increasing covalency by increasing metal ( $3 d$ )-ligand $(2 p)$ hybridization therefore more strongly reduces the metal $3 d$ character in $e_{g}$ compared to $t_{2 g}$ orbitals.

The measured and calculated Co 2p3d RIXS spectra for this series of Co compounds in Figure 13b now nicely show how the CT peaks decrease in energy with increasing covalency (decreasing charge-transfer energy $\Delta$ ) from around $11 \mathrm{eV}$ in $\mathrm{CoF}_{2}$ to around $3 \mathrm{eV}$ in CoS. These $\mathrm{CT}$ peaks correspond to, as in the Ni case discussed above, transitions from ligandcentered bonding orbitals to the metal-centered antibonding orbitals ( $t_{2 g}$ and $e_{g}$ here). It is in 
essence by matching the calculated and measured energies of these transitions that the charge-transfer energies and the covalency were determined within the CTM model. This procedure is very similar to extracting the ligand-field energy $10 \mathrm{Dq}$ by matching calculated and measured LF transition energies within the CFM model discussed in the previous section.

It is interesting to see that the intensities of the CT with respect to the LF excitations in the spectrum of the most ionic compound $\mathrm{CoF}_{2}$ in Figure 13b approximately corresponds to the CT/LF intensity ratio for the previously discussed $\mathrm{Ni}$ case in the ionic $\left[\mathrm{Ni}\left(\mathrm{H}_{2} \mathrm{O}\right)_{6}\right]^{2+}$ complex. Consistently, their ground-state charge-transfer energies $\Delta$ are similar ( $8.8 \mathrm{eV}$ for $\mathrm{CoF}_{2}$ and $6.5 \mathrm{eV}$ for $\left.\left[\mathrm{Ni}\left(\mathrm{H}_{2} \mathrm{O}\right)_{6}\right]^{2+}\right)$ and the contributions of metal $3 \mathrm{~d}$ character to their metal-centered antibonding $\mathrm{t}_{2 \mathrm{~g}} / \mathrm{e}_{\mathrm{g}}$ orbitals are similar $\left(91.7 \%\right.$ for for $\mathrm{CoF}_{2}$ and $94.5 \%$ for $\left.\left[\mathrm{Ni}\left(\mathrm{H}_{2} \mathrm{O}\right)_{6}\right]^{2+}\right)$. The investigation by Wang et al. in Figure 13 thus confirms the more qualitative study of the $\left[\mathrm{Ni}\left(\mathrm{H}_{2} \mathrm{O}\right)_{6}\right]^{2+}$ complex and demonstrates how $2 \mathrm{p} 3 \mathrm{~d}$ RIXS gives access to the important property of ground-state covalency in metal compounds by effectively probing the extent to which metal and ligand orbitals hybridize to form the metal-ligand bond.

Connecting to the previous section we finish the discussion of Figure 13 by briefly addressing the LF excitations in Figure 13c. For this Co" series the LF excitations correspond to spinallowed ${ }^{4} \Gamma \rightarrow{ }^{4} \Gamma$ transitions from the $\mathrm{Co}^{11}-\mathrm{O}_{\mathrm{h}}{ }^{4} \mathrm{~T}_{1}$ ground state. The measured spectra in Figure $13 \mathrm{c}$ clearly display how the first LF excitation in the spectrum (corresponding to the ${ }^{4} \mathrm{~T}_{1} \rightarrow{ }^{4} \mathrm{~T}_{2}$ transition for all systems in the series) shifts to lower energies from $0.85 \mathrm{eV}$ in $\mathrm{CoF}_{2}$ to 0.67 $\mathrm{eV}$ in CoS. This directly reflects the decrease of the effective calculated ligand-field strength $\left(10 \mathrm{Dq}_{\text {eff }}\right)$ from $0.92 \mathrm{eV}$ in $\mathrm{CoF}_{2}$ to $0.7 \mathrm{eV}$ in $\mathrm{CoS}$ as (Figure 13a). It needs to be mentioned here that inclusion of covalency by charge-transfer configurations within the CTM model, in contrast to the CFM model, complicates extraction of the ligand-field strength from the first LF excitation in 2p3d RIXS spectra. In the CTM model, charge transfer contributes to the $t_{2 g^{-}}$ $\mathrm{e}_{\mathrm{g}}$ splitting. Therefore, the energy of the first LF RIXS peak has to be correlated with the effective ligand-field strength $10 \mathrm{Dq} \mathrm{q}_{\text {eff }}$. This results from a combination of the ionic ligandfield strength $10 \mathrm{Dq}_{\text {ion }}$ (which is the ligand-field strength considered in the previous section where spectra were interpreted with the CFM model without inclusion of charge-transfer) and the so-called charge-transfer contribution $\Delta \mathrm{E}_{\mathrm{CT}}$ (see Figure 13a). We refer to references (De Groot and Kotani, 2008) and (Wang et al., 2017) for a more detailed description of this. 


\section{Applications of Metal K-edge RIXS}

To demonstrate the power of $1 s 2 p$ RIXS for the investigation of dilute metalloproteins and to exemplify the insights from this approach, we discuss here the $1 s 2 p$ RIXS study of the cytochrome c electron transfer protein by Kroll et al. (Kroll et al., 2014). Due to resolution and detection limits, fewer RIXS experiments have been made on metalloproteins using the $\mathrm{K} \beta$ emission channels. Here, an example due to Hadt et al. is shown for a tetranuclear $\mathrm{Co}_{4} \mathrm{O}_{4}$ cubane complex relevant for water oxidation, where 1s3p RIXS was used to understand how the metal-oxo bonds control the redox activity (Hadt et al., 2016).

\section{Electron Transfer in Cytochrome C}

Cytochrome $\mathrm{c}$ is a metalloprotein that transfers electrons between cytochrome $\mathrm{c}$ reductase and oxidase. The latter enzyme reduces dioxygen to water in a key step in aerobic cell respiration. The functional group is an iron porphyrin that changes between low-spin ferrous and ferric states. The iron has an axial iron-thioether bond that is a common motif across active sites of electron transfer proteins. In Cu enzymes the axial ligand is believed to lower the oxidation potential because the axial bond strength increases in the oxidized state while the reorganization energy is not as strongly affected. For cytochrome $c$ it has instead been proposed that the axial ligand raises the potential, e.g. based on a longer Fe-S distance in the oxidized state. The aim of the 1s2p RIXS study discussed here is to analyze the changes in ligand bonding upon reduction/oxidation and the effect on the electron transfer reaction.

It is important to note that the intense transitions in the porphyrin obscure UV/Vis probes of the iron itself. Fe L-edge XAS has been successfully used to probe the electronic structure of heme iron (Wilson et al., 2013). However, as discussed above, applying soft x-ray spectroscopy to enzymatic systems is still challenging and $1 \mathrm{~s} 2 \mathrm{p}$ RIXS is a way to obtain Ledge-like spectra. To facilitate the analysis of the electronic structure, cytochrome $c$ is compared to the model system FeTPP $(\operatorname{ImH})_{2}$ (TPP=tetraphenyl porphyrin). The main difference in the iron ligand environment between FeTPP $(\operatorname{ImH})_{2}$ and cytochrome $c$ is the replacement of one axial imidazole ligand with a thioether (Met-80), see Figure 14. 
Comparison between the two systems should give insight into the relative changes in $\sigma$ bonding when changing the oxidation state.
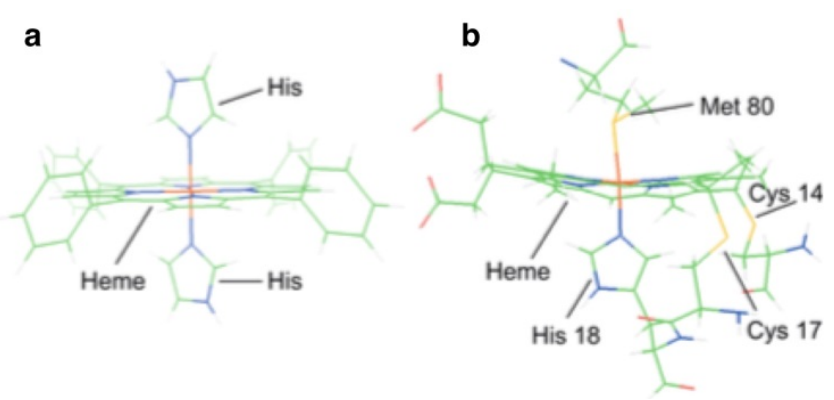

Figure 14. Molecular structures of $\mathbf{a} \operatorname{FeTPP}(\operatorname{ImH})_{2}$, and $\mathbf{b}$ the active site counterpart in cytochrome $\mathbf{c}$. Reproduced with permission from (Kroll et al., 2014). Copyright 2014 American Chemical Society.

The 1s2p RIXS spectra of FeTPP $(\operatorname{ImH})_{2}$ and cytochrome $c$ are shown in Figure 15. Unlike the spectra of the ferrous and ferric systems in Figure 5, the energy transfer axes cover the full Ledge energy range including both the $L_{3}$ - and the $L_{2}$-edges and the backgrounds from the rising edges have been subtracted. At first glance the spectra in Figure 15 have similar structure to the other low-spin complexes with cyanide and tacn ligands in Figure 5. Although the heme systems are not strictly octahedral, the $t_{2 g}$ and $e_{g}$ labels will be used for simplicity. The ferrous systems in Figure 15 therefore can be described with a single $e_{g}$ preedge XAS resonance (marked with a red line in Figures 15a and 15b), while the ferric complexes have both $t_{2 g}$ and $e_{g}$ XAS resonances (marked with green and red lines, respectively, Figures $15 c$ and 15d). For FeTPP(ImH $)_{2}$ the 1s2p RIXS CIE cut can be compared to the previously collected L-edge XAS data by Hocking et al. (Hocking et al., 2007). The comparison is similar to the ferrous tacn and ferrocyanide complexes in Figure 6 with the 1s2p RIXS CIE cut being broader than the L-edge XAS spectrum on the low-energy side, see Figure $15 \mathrm{e}$. At the same time, the relative intensity at the $\mathrm{L}_{2}$-edge is higher in the CIE cut compared to L-edge XAS. Comparison of the $1 s 2 p$ RIXS CIE cut of the ferrous model complex with that of reduced cytochrome $\mathrm{c}$ shows further differences that reflect changes in metalligand bonding, see Figure $15 f$. The first difference is a slightly reduced width of the $\mathrm{L}_{3}$-edge region in cytochrome c compared to ferrous FeTPP $(\operatorname{ImH})_{2}$, a decrease in intensity of the highenergy $L_{3}$-edge shoulder and increased intensity if the $L_{2}$-edge region. 

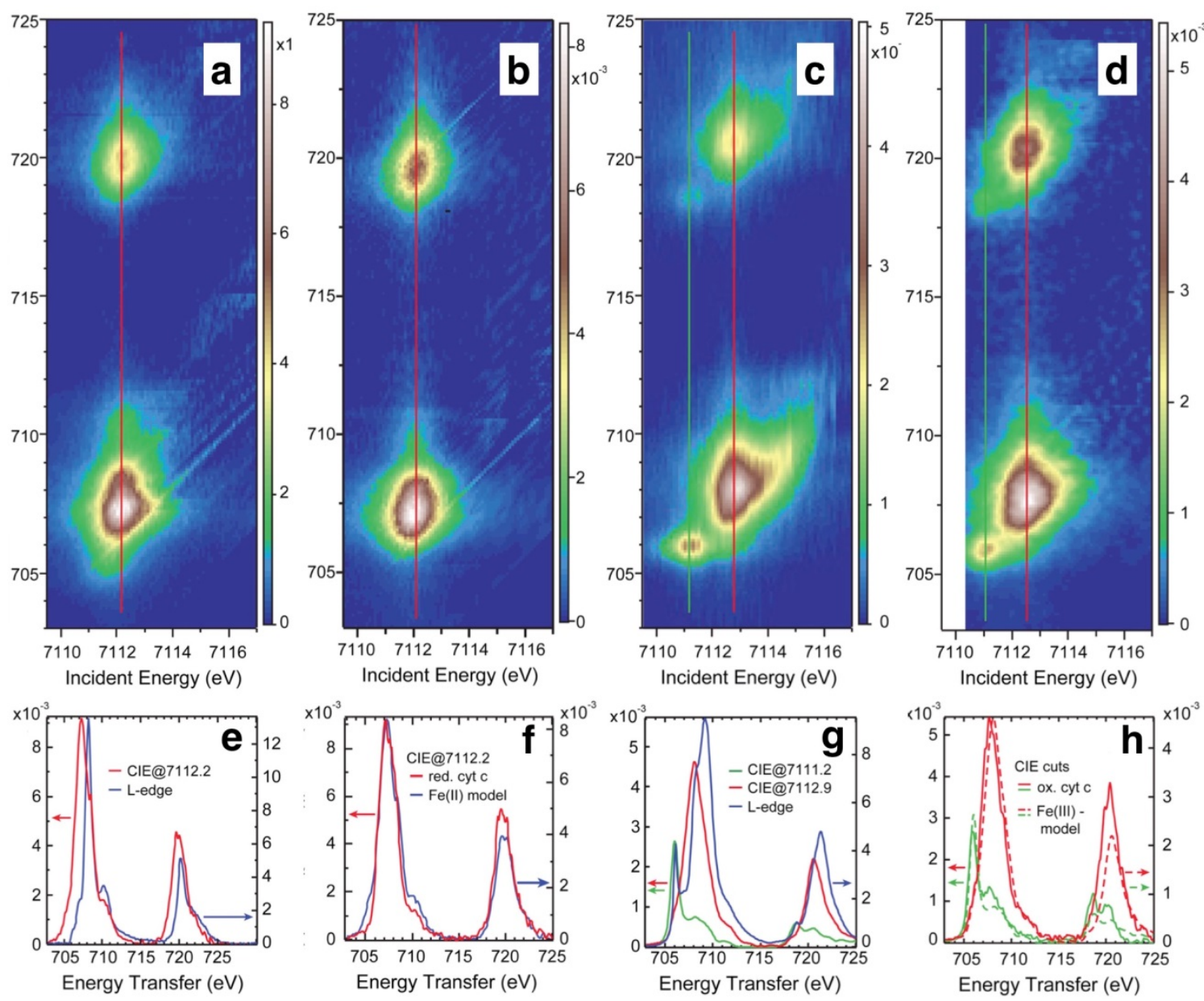

Figure 15. Rising-edge subtracted Fe 1s2p RIXS maps and corresponding Constant Incident Energy (CIE) cuts of a ferrous FeTPP $(\operatorname{ImH})_{2}$, b reduced cytochrome c, c ferric FeTPP $(\operatorname{ImH})_{2}$ and d oxidized cytochrome $\mathrm{c}$ (the red and green vertical lines are the CIE cuts). e CIE and L-edge XAS of ferrous FeTPP $(\operatorname{ImH})_{2}$, $\mathbf{C ~ C I E ~ o f ~ b o t h ~ s y s t e m s ~ i n ~ t h e ~ f e r r o u s ~ o x i d a t i o n ~ s t a t e . ~} \mathbf{g}$ CIE and L-edge XAS of ferric FeTPP $(\operatorname{ImH})_{2}$. $\mathbf{h}$ CIE of both systems in the ferric oxidation state. Adapted with permission from (Kroll et al., 2014). Copyright 2014 American Chemical Society.

The correlation between spectral differences and bonding are less straightforward than for the octahedral model complexes discussed in the first section of this chapter. CTM model calculations show that the differences are consistent with an increase in $\sigma$ covalency for the Fe-S bond in cytochrome c compared to the Fe-N bond in imidazole. In the ferric complexes, both the $t_{2 g}$ and the $e_{g}$ XAS resonances are present and can be analyzed, see Figure 15g-h. Analysis with the CTM model together with DFT calculations shows that methionine in cytochrome $\mathrm{c}$ is a stronger $\sigma$ donor than imidazole also in the ferric state. More importantly, there is a higher degree of covalency for the ferric states relative to the ferrous states, which translates to stronger metal-ligand bonds. The presence of the axial S(Met) bond thus lowers the oxidation potential due to the stronger Fe-S bond in the oxidized state. This RIXS-based 
analysis shows how the axial ligand controls the electron transfer properties in cytochrome $c$ in a similar way as in the Cu electron transfer proteins.

\section{Cubane-like Co Cluster of Relevance for Water Oxidation}

The generation and storage of solar fuels is highly dependent on the efficiency of the oxygen evolution reaction where water is oxidized to oxygen $\left(\mathrm{O}_{2}\right)$. To develop better catalysts requires insight into the electronic and geometric structure of the active sites. An intensely studied system is the cobalt phosphate, which comprises a $\mathrm{Co}_{4} \mathrm{O}_{4}$ cubane as its basic structural element. The formation of high-valent states is a key factor for many oxygenevolution catalysts and for the cobalt phosphate system the proposed key species is the highly oxidized $\mathrm{Co}^{\mathrm{IV}}$ and this calls for better understanding the electronic structure of $\mathrm{Co}^{\mathrm{IV}}$ species. However, $\mathrm{Co}^{\mathrm{IV}}$ often is a minority species against a large background of Co $\mathrm{C}^{\text {II }}$ because only a minor fraction of the sample is redox active. This represents a severe experimental challenge and limitation in characterizing $\mathrm{Co}^{\mathrm{IV}}$ in catalytically active cobalt phosphate.

A number of $\mathrm{CO}_{4} \mathrm{O}_{4}$ cubane systems have been designed to address this challenge, among them $\mathrm{Co}_{4} \mathrm{O}_{4}(\mathrm{OAc})_{4}(\mathrm{py})_{4}(\mathrm{OAc}=$ acetate and py=pyridine). This system can be characterized both in the neutral $\mathrm{Co}_{4}{ }_{4}$ and oxidized $\mathrm{CO}^{\mathrm{IV}} \mathrm{CO}^{\prime \prime \prime}{ }_{3}$ states, and it also exhibits catalytic activity after a second oxidation step. 1s3p RIXS has been used to probe cobalt oxides (AI Samarai et al., 2016), and was also applied to the reduced and one-electron oxidized $\mathrm{Co}_{4} \mathrm{O}_{4}$ complexes by Hadt et al., see Figure 16 (Hadt et al., 2016). The 1s3p RIXS process gives final states with $3 p$ core holes and appropriate cuts through the RIXS map correspond to metal M-edge $(3 p \rightarrow 3 d)$ absorption. 

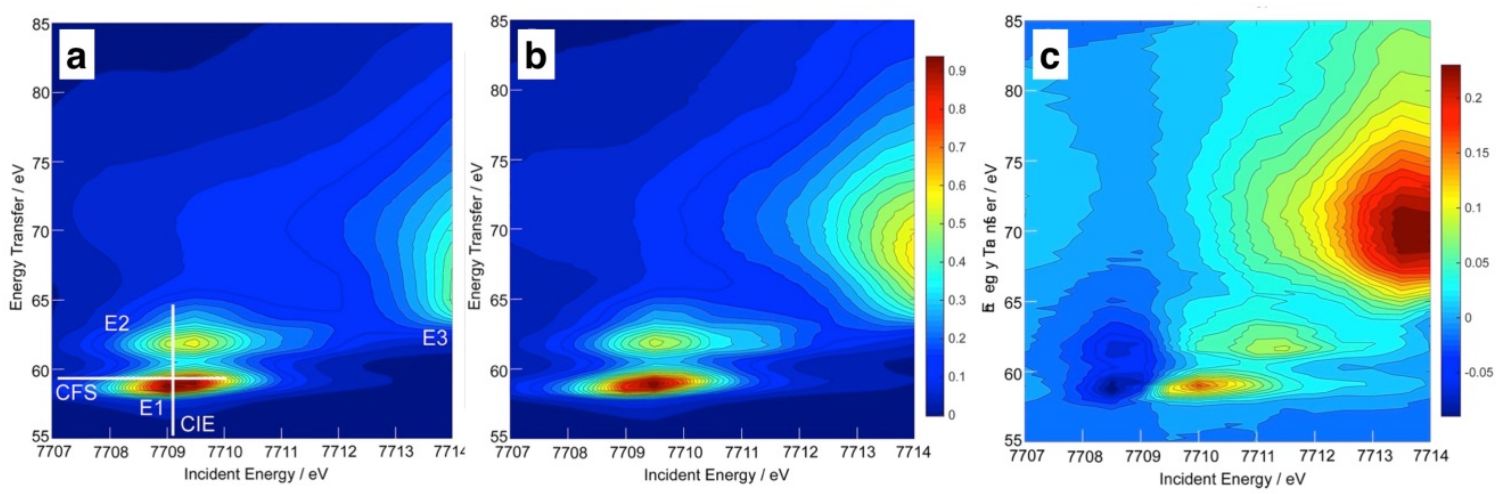

Figure 16. Co $1 s 3 p$ RIXS maps for $\mathrm{Co}_{4} \mathrm{O}_{4}(\mathrm{OAc})_{4}(\mathrm{py})_{4}$. a Reduced $\mathrm{Co}^{\prime \prime \prime}{ }_{4}$ complex where CFS denotes lines with Constant Final States and E1, E2 and E3 label the regions with high intensities, b Oxidized $\mathrm{Co}^{\text {IV }} \mathrm{Co}_{3}{ }_{3}$ complex, c Extracted Co ${ }^{\text {IV }}$ spectrum (oxidized complex - (3/4) reduced complex). Reproduced with permission from (Hadt et al., 2016). Copyright 2016 American Chemical Society.

To isolate the signal from the high-valent $\mathrm{Co}^{\text {IV }}$ species, the contributions from three Co ${ }^{\text {III }}$ ions are removed from the spectrum of the oxidized species. In the extracted Co ${ }^{\mathrm{IV}}$ RIXS spectrum there are three resonances with the largest intensity changes occurring at energies approaching the rising edge (Figure 16c). To analyze the Co ${ }^{\mathrm{IV}}$ RIXS spectrum, CIE cuts at certain $\mathrm{K}$ pre-edge resonances can be used, see Figure 17. Co ${ }^{\mathrm{IV}}$ is a $3 d^{5}$ system with $\mathrm{t}_{2 \mathrm{~g}}{ }^{5} \mathrm{eg}_{\mathrm{g}}{ }^{0}$ ground state. The $\mathrm{K}$ pre-edge has the same $t_{2 g}$ and $e_{g}$ resonances, labeled $A$ and $B$ in Figure 17 , as previously discussed for the low-spin ferric systems. The $\mathrm{e}_{\mathrm{g}}$ resonance contains several $t_{2 g}{ }^{5} e_{g}{ }^{1}$ final states, with different valence spin multiplicities and relative orientation of $t_{2 g}$ hole and $e_{\mathrm{g}}$ electron, ${ }^{1,3}\left(\mathrm{~T}_{1 \mathrm{~g}}, \mathrm{~T}_{2 \mathrm{~g}}\right)$, as outlined in Figure 4. The CIE cut in this region shows clear signatures of $\mathrm{Co}^{\mathrm{IV}}$, where the $3 p$ hole is used as a sensitive probe of the local electronic structure of the oxidized Co ion. 

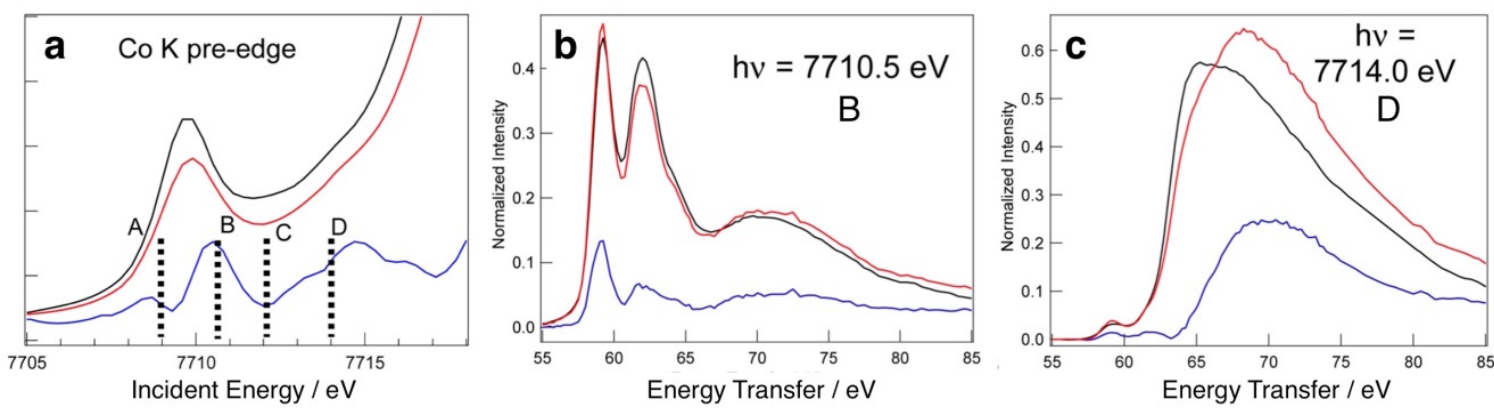

Figure 17. a Co K pre-edge absorption spectra of $\mathrm{CO}_{4} \mathrm{O}_{4}(\mathrm{OAC})_{4}(\mathrm{py})_{4}$ and constant incident energy cuts of the $1 \mathrm{~s} 3 p$ RIXS planes at $\mathbf{b}$ resonance $B$ and $\mathbf{c}$ at resonance $\mathrm{D}$. In all graphs the reduced $\mathrm{Co}{ }_{4}{ }_{4}$ complex is shown with black lines, the oxidized $\mathrm{CO}^{\mathrm{IV}} \mathrm{CO}^{\mathrm{III}}{ }_{3}$ complex with red lines and the extracted $\mathrm{Co}^{\mathrm{IV}}$ spectrum with blue lines. Adapted with permission from (Hadt et al., 2016). Copyright 2016 American Chemical Society.

Another interesting region appears at $\sim 5 \mathrm{eV}$ above the pre-edge, labeled $\mathrm{D}$ in Figure 17. Intensity there is present for both reduced and oxidized complexes but it increases in the latter, contrary to the expected shift to higher energies of the rising edge. This resonance is assigned to a non-local transition where the $1 \mathrm{~s}$ electron is transferred to a neighboring Co atom. In the final state of this resonance the $3 p$ hole and the additional $3 d$ electron are on different sites and the resulting interatomic interactions probe the oxo-mediated metalmetal interactions that are essential for determining redox properties and activity. With the use of DFT calculations it could be shown that redox activity is linearly related to covalency of the $\mathrm{Co}^{\mathrm{IV}}$ oxo bonding and that these interactions tune the oxidation potential over hundreds of millivolts. 1s3p RIXS therefore offers a sensitive probe of these interactions and can thus be used to analyze how modifications of the catalyst could affect its catalytic activity.

\section{Time-resolved RIXS for characterizing transient reaction intermediates}

With this we leave behind some of the most recent investigations of steady-state bonding and structure in transition-metal complexes and metalloproteins and continue exploring new aspects of RIXS, the study of the time evolution in optically excited systems. For this we proceed to applications of time-resolved RIXS for the detection and characterization of transient reaction intermediates. RIXS is implemented in a pump-probe scheme where an optical laser pulse (pump pulse) photo-excites the system and triggers a photo-reaction, and 
a time-delayed x-ray pulse is used to probe the state of the system in a stroboscopic fashion. By repeating the measurement for different time delays, the evolution of the valence electronic structure is followed in time.

The time-resolved RIXS experiments on transition-metal complexes discussed here build on the more established time-resolved XAS and XES investigations at SR facilities (Bressler and Chergui, 2004, Chergui and Collet, 2017, Cordones et al., 2018, Hong et al., 2015, March et al., 2015, Chen, 2005, Kim et al., 2015a, Milne et al., 2014) and they complement recent femtosecond XAS, XES and solution scattering experiments of metal complexes and metalloproteins with hard x-rays from XFELs.(Canton et al., 2015, Kim et al., 2015b, Levantino et al., 2015, Mara et al., 2017, Miller et al., 2017, Zhang et al., 2014, Zhang and Gaffney, 2015)

At the time of publication of this chapter, time-resolved RIXS investigations in general and of transition-metal complexes in particular are scarce and one of the reasons is the current lack of suitably bright short-pulse $\mathrm{x}$-ray sources.

Of the pioneering time-resolved RIXS studies of transition-metal systems at SR sources, (Penfold et al., 2018, Vankó et al., 2013, Vankó et al., 2015) we briefly discuss in more detail the exemplary time-resolved K-edge RIXS study of Fe complexes in solution by Vankó et al. (Vankó et al., 2015) This investigation addresses the nanosecond time scale defined by the lifetime of the investigated electronic excited state of the system and the picosecond temporal resolution achievable at SR sources well matches this case. The unprecedented peak brilliance at XFELs with femtosecond $x$-ray pulses has recently enabled a number of time-resolved soft x-ray RIXS studies of molecular systems in solution (Eckert et al., 2017, Jay et al., 2018b, Kunnus et al., 2016b, Wernet et al., 2015) With a temporal resolution in the femtosecond domain these investigations address the time scale of nuclear motion in chemical reactions and the studies by Jay et al. and Wernet et al. are used to demonstrate the possibility to follow in time the evolution of ligand-field effects, symmetry, chargetransfer and, as a new aspect in this section, the interactions of frontier orbitals in metal complexes with femtosecond time-resolved $2 p 3 d$ RIXS.

We note that for the femtosecond RIXS experiments discussed here, the $\mathrm{x}$-ray pulse durations and the temporal resolution of the experiment are on the order of $100 \mathrm{fs}$ and therefore significantly larger than $1 s$ and $2 p$ core-hole lifetimes of the order of one 
femtosecond. When the x-ray pulse duration approaches the core-hole lifetime, however, non-linear x-ray effects can be observed (Beye et al., 2013, Kroll et al., 2018, Rohringer et al., 2012, Yoneda et al., 2015, Bencivenga et al., 2015).

We start with the time-resolved $1 s 2 p$ RIXS investigation of $\mathrm{Fe}^{\|}-\mathrm{O}_{\mathrm{h}}$ in the polypyridyl compound $\left[\mathrm{Fe}(\text { terpy })_{2}\right]^{2+}$ in Figure 19 performed by Vankó et al. at a SR source with a temporal resolution on the order of tens of picoseconds (for complementary L-edge XAS studies of the same and related systems see Hong et al., 2015).

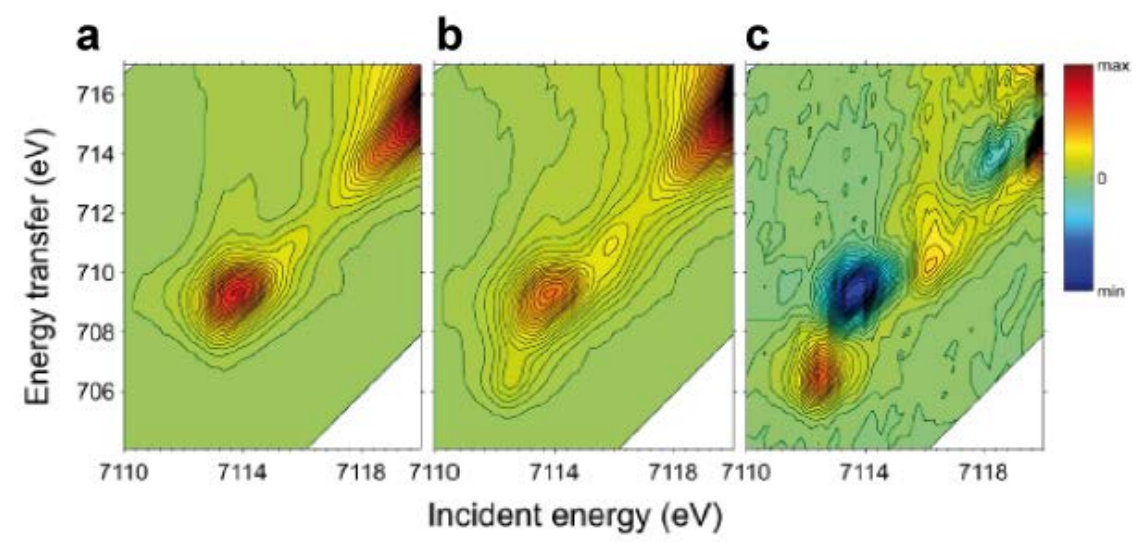

Figure 18. Fe 1s2p RIXS maps of $\mathrm{Fe}^{\prime \prime}-\mathrm{O}_{\mathrm{h}}$ in $\left[\mathrm{Fe}(\text { terpy })_{2}\right]^{2+}$ in aqueous solution (incident-energy range of $\mathrm{K}$ pre-edge excitations) in a its low-spin $\left({ }^{1} \mathrm{~A}_{1 \mathrm{~g}}\right)$ ground state, in $\mathbf{b}$ the photo-induced quintet state $\left({ }^{5} \mathrm{E}\right)$ and $\mathbf{c}$ the difference of $\mathbf{b}$ minus a (red: increase, blue: decrease of intensity). Adapted with permission from (Vankó et al., 2015). Copyright 2015 American Chemical Society.

Optical excitation of the low-spin ground state of this system with $a t_{2 g}{ }^{6}$ configuration at a wavelength of $515 \mathrm{~nm}$ corresponds to a MLCT excitation that is followed by intricate femtosecond dynamics that were not resolved in this study. Relaxation from the optically excited state ultimately leads to the high-spin quintet state of $\left[\mathrm{Fe}(\mathrm{terpy})_{2}\right]^{2+}$ with $\mathrm{t}_{2 \mathrm{~g}}{ }^{4} \mathrm{eg}_{\mathrm{g}}{ }^{2}$ configuration before this quintet state decays back to the ground state. Vankó et al. determine the lifetime of the quintet state to $2.6 \mathrm{~ns}$ and this justifies why it can be effectively characterized with the picosecond temporal resolution of the SR experiment.

The Fe 1s2p RIXS spectra taken at the $\mathrm{K}$ pre-edges of ground- and photo-induced high-spin quintet states of $\left[\mathrm{Fe}(\text { terpy })_{2}\right]^{2+}$ are compared in Figure 19. The apparent differences between the spectra in Figure 19a and 19b are highlighted by their difference in Figure 19c. This can be conceptually understood by comparison with the differences between the Fe 1s $2 p$ RIXS spectra of ferrous and ferric systems in Figure 5. For the low-spin $\left(\mathrm{t}_{2 g}{ }^{6} \mathrm{e}_{\mathrm{g}}{ }^{0}\right)$ case, the $\mathrm{K}$ preedge exclusively consists of a $1 \mathrm{~s} \rightarrow \mathrm{e}_{\mathrm{g}} \mathrm{XAS}$ resonance at an incident energy of $7113 \mathrm{eV}$ and an 
energy transfer of $709 \mathrm{eV}$ (the rising K-edge is visible at $7118 \mathrm{eV}$ and $714 \mathrm{eV}$ and was not subtracted from the spectra here). In the photo-induced high-spin $\left(\mathrm{t}_{2 \mathrm{~g}}{ }^{4} \mathrm{e}_{\mathrm{g}}{ }^{2}\right)$ case a new resonance at low energies corresponding to $1 \mathrm{~s} \rightarrow \mathrm{t}_{2 \mathrm{~g}}$ transitions occurs at 7112-7113 eV and $707 \mathrm{eV}$. Based on these differences, with additional information form XAS and XES, and with calculations the authors characterized in detail the quintet state of $\left[\mathrm{Fe}(\text { terpy })_{2}\right]^{2+}$. They also used time-resolved extended $x$-ray absorption fine structure spectroscopy (EXAFS) to characterize its geometric structure and they applied time-resolved $x$-ray diffuse scattering to probe the reaction of the solvent to the electronic excitation of the solute. The study by Vankó et al. therefore demonstrates the power of combining time-resolved RIXS as a probe of the electronic structure of metal complexes with time-resolved geometric structure probes.

Now addressing time-resolved metal L-edge RIXS, we come back to the case of Fe $\mathrm{F}^{\text {III- }} \mathrm{O}_{\mathrm{h}}$ in the ferricyanide $\left[\mathrm{Fe}^{\text {III }}(\mathrm{CN})_{6}\right]^{3-}$ complex which was treated in detail in the first section of this chapter in terms of steady-state $1 s 2 p$ and $2 p 3 d$ RIXS. Here we discuss with Figure 19 the investigation of the same molecule but now after photoexcitation and using time-resolved $2 p 3 d$ RIXS with femtosecond soft $x$-ray pulses of the $x$-ray free-electron laser LCLS at the SLAC National Accelerator Laboratory by Jay et al. (Jay et al., 2018b). 

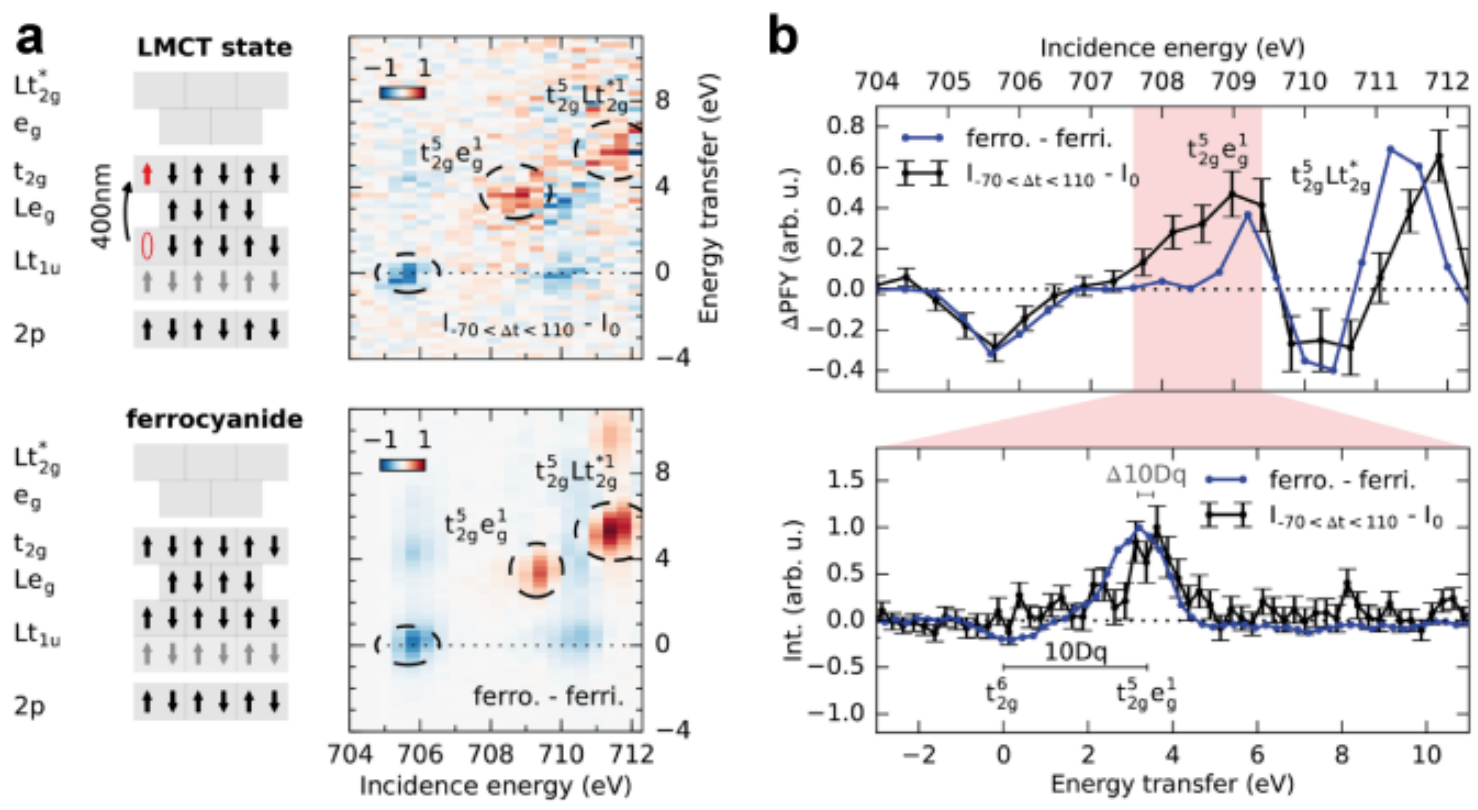

Figure 19. a Electron configurations and Fe $\mathrm{L}_{3}$-RIXS maps of top: LMCT excited state of ferricyanide $\left(\mathrm{Fe}{ }^{\prime \prime \prime}-\mathrm{O}_{\mathrm{h}}\right.$ in $\left.\left[\mathrm{Fe}(\mathrm{CN})_{6}\right]^{3-}\right)$ and bottom: ground-state ferrocyanide $\left(\mathrm{Fe}{ }^{\prime \prime}-\mathrm{O}_{\mathrm{h}}\right.$ in $\left.\left[\mathrm{Fe}(\mathrm{CN})_{6}\right]^{4}\right)$ (the RIXS maps are difference maps of the given systems with respect to the ground state of ferricyanide, top: LMCT of ferricyanide for the pump-probe delay interval of -70-110 fs minus ground-state ferricyanide, bottom: ferrocyanide minus ferricyanide, blue: decrease of intensities, red: increase, intensities normalized to one/minus one at maximum/minimum, experimental bandwidth $1 \mathrm{eV}$, temporal resolution $180 \mathrm{fs}$ ). All measurement performed in aqueous solution. $\mathbf{b}$ Top: $\mathrm{Fe} \mathrm{L}_{3} \mathrm{x}$-ray absorption spectra of LMCT of ferricyanide minus ground state ferricyanide (black) and of ferrocyanide minus ferricyanide (blue, both ground states), $\triangle \mathrm{PFY}$ stands for partial-fluorescence yield corresponding to displaying intensities integrated along the energy transfer axis of the RIXS maps in a versus incident photon energy (integrated for energy transfers from 2 to $7.5 \mathrm{eV}$ ). Bottom: Fe $\mathrm{L}_{3}-\mathrm{RIXS}$ spectra at the $2 p \rightarrow e_{g}$ resonance (vertical cuts through the RIXS maps in a corresponding to displaying intensities at that resonance at 707.6-709.4 eV, normalized to one at maximum). Reproduced with permission from (Jay et al., 2018b). Copyright 2018 American Chemical Society.

The complex dynamics of ferricyanide following optical excitation range from the femto- to the nanosecond range and Jay et al. concentrated on characterizing the initial photo-excited LMCT state by analyzing RIXS data for temporally overlapping pump and probe pulses (measurements around zero pump-probe delay). The femtosecond temporal resolution was thus used to isolate the transient intermediate LMCT state to characterize the optically induced charge redistribution. The study can therefore be regarded as a benchmark case for how transient charge distributions in transition-metal complexes can be studied with RIXS. Optical excitation of ferricyanide at the employed wavelength of $400 \mathrm{~nm}$ corresponds to an LMCT excitation assigned to the $\mathrm{Lt}_{1 \mathrm{u}} \rightarrow \mathrm{t}_{2 \mathrm{~g}}$ one-electron transition (Figure 19a) where the ligand-dominated $\mathrm{Lt}_{1 \mathrm{u}}$ orbital is due to the interaction of the $\mathrm{CN} 5 \sigma$ and Fe $3 \mathrm{~d}$ orbitals (see also Figure 2). The changes upon LMCT excitation of ferricyanide are compared to the 
changes upon going from $\mathrm{Fe}^{\mathrm{III}}-\mathrm{O}_{\mathrm{h}}$ in ground-state ferricyanide to $\mathrm{Fe}^{\prime \prime}-\mathrm{O}_{\mathrm{h}}$ in ground-state ferrocyanide $\left(\mathrm{Fe}^{\|}-\mathrm{O}_{\mathrm{h}}\right.$ in $\left.\left[\mathrm{Fe}^{\prime \prime}(\mathrm{CN})_{6}\right]^{4-}\right)$. In this way the effect of populating the $t_{2 \mathrm{~g}}$ orbital can be studied independently for the case with ligand $\mathrm{Lt}_{1 \mathrm{u}}$ hole (in the LMCT state of ferricyanide) and without (in ferrocyanide). The changes in the Fe 2p3d RIXS maps of ferricyanide upon LMCT excitation and upon going to ferrocyanide are displayed in Figure 19a. Closing the $\mathrm{t}_{2 \mathrm{~g}}$ shell $\left(t_{2 g}{ }^{5} \rightarrow t_{2 g}{ }^{6}\right)$ depletes the $2 p \rightarrow t_{2 g}$ XAS resonance at 705-706 eV for both LMCT of ferricyanide and ferrocyanide (with respect to ground-state ferricyanide, Figure 19a). At the same time the LF excitations ( $t_{2 g} \rightarrow e_{g}$ transitions at $4 \mathrm{eV}$ ) and the CT excitations $\left(t_{2 g} \rightarrow \mathrm{Lt}_{2 g}\right.$ * transitions at 5-6 eV) that are characteristic of $\mathrm{Fe}^{11}-\mathrm{O}_{\mathrm{h}}$ with $\mathrm{t}_{2 \mathrm{~g}}{ }^{6} \mathrm{e}_{\mathrm{g}}{ }^{0}$ configuration appear in both systems (where $\mathrm{Lt}_{2 \mathrm{~g}}{ }^{*}$ is a ligand-centered antibonding orbital that can also be denoted $\pi^{*}$, see the section on the principles of RIXS for $3 d$ transition-metal complexes earlier in this chapter). Differences between the two systems are already apparent when inspecting in detail the spectral widths and positions of the $t_{2 g} \rightarrow e_{g}$ transitions in the RIXS maps in Figure 19a and they are emphasized by the Fe $L_{3}$ absorption and RIXS spectra in Figure 19b. The $2 p \rightarrow e_{g}$ resonance in the absorption spectrum of LMCT of ferricyanide is shifted to lower energies to about $707.5 \mathrm{eV}$ compared to ferrocyanide (709 eV, Figure 19b top). This may indicate a stabilization of the core-excited $2 \mathrm{p}^{5} \mathrm{t}_{2 \mathrm{~g}}{ }^{6} \mathrm{e}_{\mathrm{g}}{ }^{1}$ states in LMCT of ferricyanide compared to ferrocyanide due to a higher electron density at the Fe site. Jay et al. also find that the amount of Fe-ligand $\pi$-back-donation is similar for LMCT of ferricyanide and ferrocyanide with Fe $3 d$ contributions to the $\pi$-accepting ligand $\mathrm{Lt}_{2 \mathrm{~g}}{ }^{*}$ orbital of around $11 \%$ for both. The higher electron density at Fe with a similar amount of $\pi$-back-donation therefore points to an enhanced ligand-Fe $\sigma$-donation in LMCT of ferricyanide compared to ferrocyanide. And indeed they find enhanced covalency in LMCT of ferricyanide compared to ferrocyanide due to stronger mixing of $\mathrm{Fe} \mathrm{e}_{\mathrm{g}}$ and ligand-dominated Le $\mathrm{e}_{\mathrm{g}}$ orbitals (the contribution of $\mathrm{Fe} 3 \mathrm{~d}$ character in the Le orbital is increase from $14 \%$ in ferrocyanide to $18 \%$ in LMCT of ferricyanide). With calculated electron density differences the authors traced this back to the characteristic difference between LMCT of ferricyanide and ferrocyanide: The ligand hole in the non-bonding ligand-dominated $\mathrm{Lt}_{1 \mathrm{u}}$ orbital in $\mathrm{LMCT}$ of ferricyanide reduces the Coulomb repulsion with the $\sigma$-donating $L_{\mathrm{g}}$ bonding orbital thereby enabling it to hybridize more strongly with the Fe $\mathrm{e}_{\mathrm{g}}$ orbitals. Confirming this explanation, the LF excitation energies $\left(t_{2 g} \rightarrow e_{g}\right.$ transitions around $4 \mathrm{eV}$ ) in the $2 \mathrm{p} 3 \mathrm{~d}$ RIXS spectra in Figure 19b (bottom) are found to blue-shift by about $0.3 \mathrm{eV}$ from LMCT of ferricyanide to ferrocyanide revealing an 
approximately $10 \%$ increase of the ligand-field strength, $10 \mathrm{Dq}$, as a result of increased covalent $\sigma$ interactions in LMCT of ferricyanide compared to ferrocyanide.

We now turn away from the study of transient charge transfer to the case where optical excitation triggers bond breaking still keeping the aspect of using LF excitations to characterize transient states of transition-metal complexes. This is done with the timeresolved femtosecond Fe 2p3d RIXS investigation of the ligand exchange dynamics of $\mathrm{Fe}(\mathrm{CO})_{5}$ in solution at the LCLS XFEL by Wernet et al. The main results of this study that are relevant in the context of this chapter are shown in Figure 20. This example is addressed here only briefly as it has been the subject of a recent book chapter already in reference (Wernet, 2017) and was detailed in (Kunnus et al., 2016b). 
a
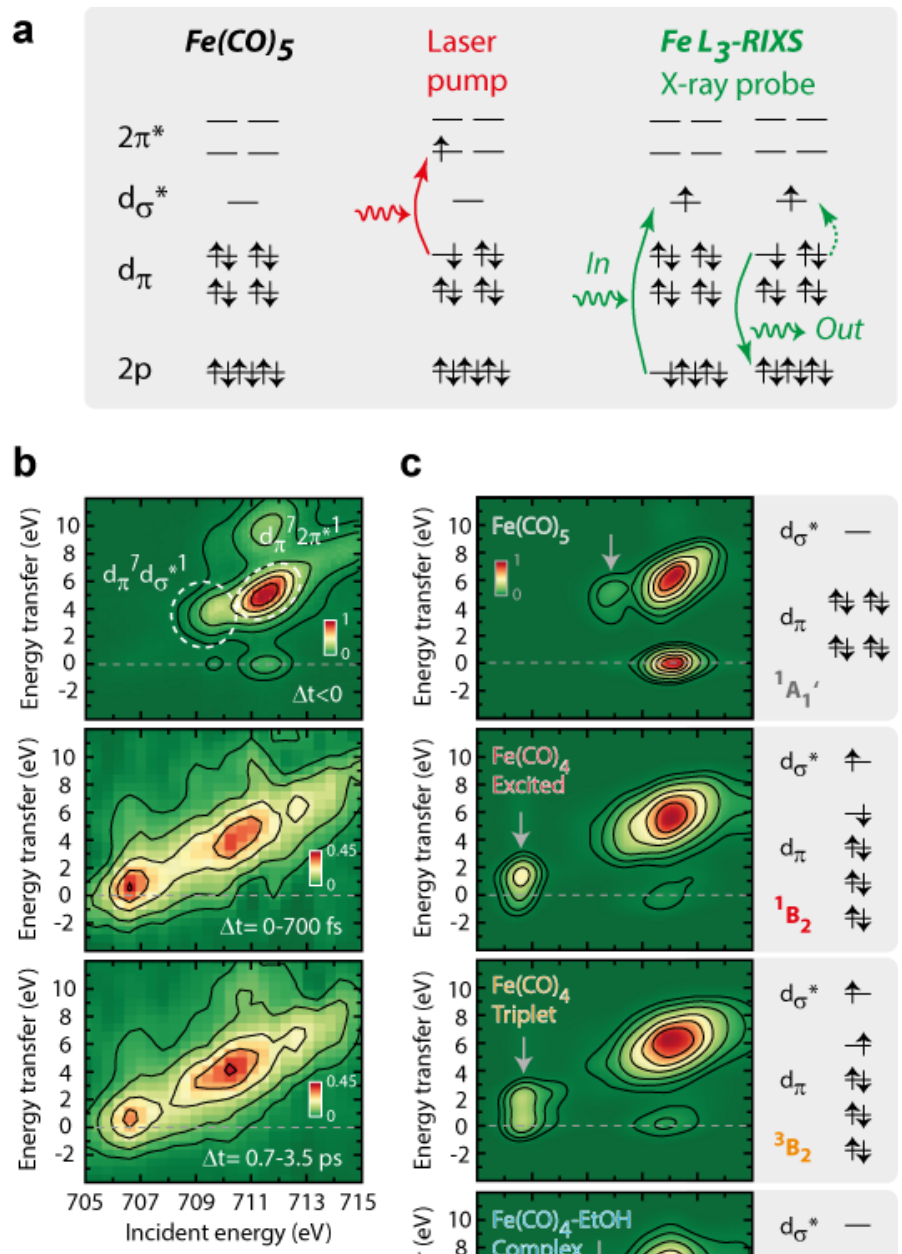

C

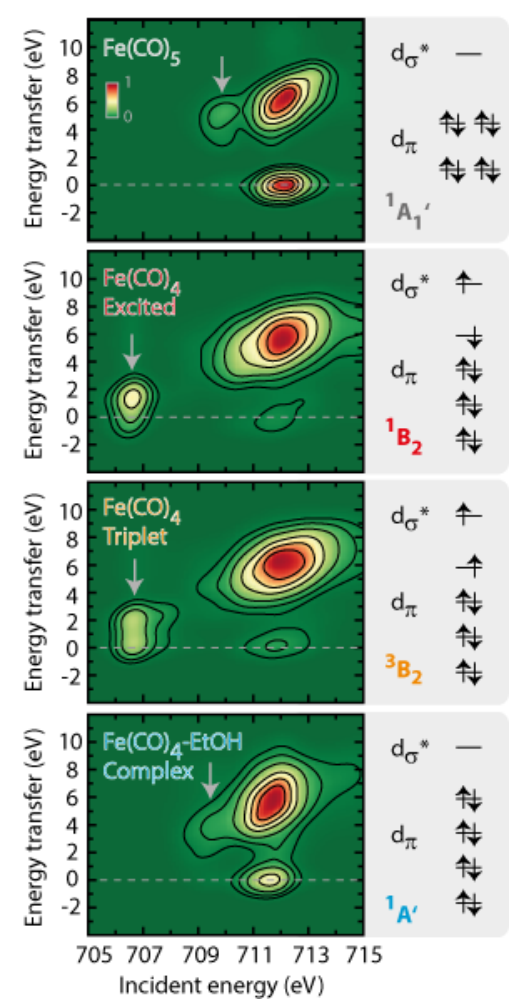

Figure 20. a Molecular-orbital energy diagram of ground-state $\mathrm{Fe}(\mathrm{CO})_{5}$ for $\mathrm{Fe} 2 \mathrm{p}$ and $\mathrm{d}_{\pi} / \mathrm{d}_{\sigma}{ }^{*}$ and ligand $2 \pi^{*}$ orbitals with "Optical pump" corresponding to a metal to ligand charge transfer (MLCT) excitation triggering dissociation, and with one-electron transitions of incident (“In", $2 p \rightarrow d_{\sigma}{ }^{*}$ excitation) and emitted ("Out") x-ray radiation for Fe L-edge RIXS (orbital symmetry along the Fe-CO bonds, asterisks mark antibonding orbitals). The dashed arrow indicates the resulting ligand-field (LF) excitations $\left(\mathrm{d}_{\pi} \rightarrow \mathrm{d}_{\sigma}{ }^{*}\right.$ transitions). b Measured $\mathrm{Fe} \mathrm{L}_{3}$-RIXS maps of ground-state $\mathrm{Fe}(\mathrm{CO})_{5}$ and transient intermediates for pump-probe delay intervals of 0-700 fs and 0.7-3.5 ps (intensities encoded in color and normalized to one at maximum, experimental bandwidth $1 \mathrm{eV}$, temporal resolution $300 \mathrm{fs}$ ). c Calculated $\mathrm{Fe} \mathrm{L}_{3}$-RIXS maps and electronic configurations of the given species $\left(d_{\pi} \rightarrow d_{\sigma}{ }^{*}\right.$ transitions marked by arrows, they result from $2 p \rightarrow$ LUMO excitation where the LUMO can be $d_{\sigma}{ }^{*}$ or $d_{\pi}$, depending on the electron configuration). Adapted from (Wernet et al., 2015).

Optical excitation of $\mathrm{Fe}(\mathrm{CO})_{5}$ at a wavelength of $266 \mathrm{~nm}$ corresponds to a MLCT excitation. The corresponding $d_{\pi} \rightarrow 2 \pi^{*}$ transition from the Fe-centered $d_{\pi}$ HOMO to the ligandcentered $2 \pi^{*}$ anti-bonding orbital (Figure 20a) induces dissociation of one Fe-CO bond on a 
time scale of $100 \mathrm{fs}$ and the bonding capabilities of the coordinatively unsaturated reactive transient intermediate $\mathrm{Fe}(\mathrm{CO})_{4}$ are probed by $2 \mathrm{p} 3 \mathrm{~d}$ RIXS for various time delays after excitation. Here we concentrate on the lowest-energy LF excitations which in $\mathrm{Fe}(\mathrm{CO})_{5}$, $\mathrm{Fe}(\mathrm{CO})_{4}$ and the related transient intermediates correspond to $d_{\pi} \rightarrow d_{\sigma}{ }^{*}$ transitions. These can be probed at the lowest-energy resonance in the $\mathrm{L}_{3}$-edge absorption spectrum via $2 p \rightarrow d_{\sigma} *$ transitions where $d_{\sigma} *$ is the LUMO (dashed arrow in Figure 20a, right). The Fe 2p3d RIXS map of intact Fe(CO) $)_{5}$ is shown in Figure 20b (top panel) and the $d_{\pi} \rightarrow d_{\sigma}{ }^{*}$ transitions are marked by a dashed circle according to their final state $d_{\pi}{ }^{7} d_{\sigma}{ }^{11}$ configuration (for the shown negative time delays, probe pulses arrive before pump pulses leaving the sample as unexcited $\left.\mathrm{Fe}(\mathrm{CO})_{5}\right)$. The steady-state Fe $2 \mathrm{p} 3 \mathrm{~d}$ RIXS spectra of $\mathrm{Fe}(\mathrm{CO})_{5}$ were studied in detail before (Suljoti et al., 2013). The Fe 2p3d RIXS maps in Figure 20b for pump-probe time-delay intervals of 0-700 fs and 0.7-3.5 ps clearly reveal a decrease of the $d_{\pi} \rightarrow d_{\sigma}{ }^{*}$ transition energies from 3-4 eV in $\mathrm{Fe}(\mathrm{CO})_{5}$ to $0-1 \mathrm{eV}$ for the transient intermediates as probed at times up to 3.5 ps where $\mathrm{Fe}(\mathrm{CO})_{4}$ is the most abundant species. This decrease can be understood as a direct indication for how the HOMO-LUMO energy difference decreases due to an overall decrease of covalent interactions in the system as one $\mathrm{CO}$ dissociates from $\mathrm{Fe}(\mathrm{CO})_{5}$ to form $\mathrm{Fe}(\mathrm{CO})_{4}$. With the related decrease of the LUMO $\mathrm{d}_{\sigma}{ }^{*}$ energy (the HOMO energy increases accordingly) the $2 p \rightarrow L U M O / d_{\sigma}{ }^{*}$ resonance energy decreases from $710 \mathrm{eV}$ in $\mathrm{Fe}(\mathrm{CO})_{5}$ to $706-707 \mathrm{eV}$ for $\mathrm{Fe}(\mathrm{CO})_{4}$ as seen in the measured RIXS maps. Changes of the measured RIXS intensities for 0-700 fs compared to 0.7-3.5 ps in Figure 20b indicate that the populations of respective species vary with time (see e.g. the decrease of the $d_{\pi} \rightarrow d_{\sigma} *$ transitions). These changes can be described with a kinetic model and, with assignments of the measured RIXS intensities to calculated spectra of characteristic species, possible reaction pathways can be inferred (Kunnus et al., 2016b, Wernet, 2017, Wernet et al., 2015). The most abundant species found in this study are singlet- and triplet-state $\mathrm{Fe}(\mathrm{CO})_{4}$ and the ligated structure $\mathrm{Fe}(\mathrm{CO})_{4}$-EtOH where an ethanol solvent molecule ligates with singlet-state $\mathrm{Fe}(\mathrm{CO})_{4}$ (Figure 20c). The calculated Fe 2p3d RIXS maps of these species detail how the $d_{\pi} \rightarrow d_{\sigma}{ }^{*}$ and $2 p \rightarrow L U M O / d_{\sigma}{ }^{*}$ transition energies vary depending on the bonding situation of $\mathrm{Fe}(\mathrm{CO})_{4}$ (gray arrows in Figure 20c): Compared to $\mathrm{Fe}(\mathrm{CO})_{5}$, the energies of these transition decrease, as observed in experiment, for excited-state singlet- and triplet-state $\mathrm{Fe}(\mathrm{CO})_{4}$ due to the decreasing covalent interactions when a ligand is lost. Ligation of $\mathrm{Fe}(\mathrm{CO})_{4}$ with ethanol 
in $\mathrm{Fe}(\mathrm{CO})_{4}-\mathrm{EtOH}$, in turn, increases orbital interactions and correspondingly restores the energies of the $d_{\pi} \rightarrow d_{\sigma}{ }^{*}$ and $2 p \rightarrow L U M O / d_{\sigma}{ }^{*}$ transitions as in $\mathrm{Fe}(\mathrm{CO})_{5}$.

It is interesting to note that the ligation capability of $\mathrm{Fe}(\mathrm{CO})_{4}$ is found to be determined by the population of the $\mathrm{LUMO} / \mathrm{d}_{\sigma}{ }^{*}$. Singlet-state $\mathrm{Fe}(\mathrm{CO})_{4}$ with an unpopulated $\mathrm{LUMO} / \mathrm{d}_{\sigma}{ }^{*}$ ligates with $\mathrm{CO}$ to form $\mathrm{Fe}(\mathrm{CO})_{5}$ or with ethanol to form $\mathrm{Fe}(\mathrm{CO})_{4}$ - $\mathrm{EtOH}$, while excited singletstate $\mathrm{Fe}(\mathrm{CO})_{4}$ and triplet-state $\mathrm{Fe}(\mathrm{CO})_{4}$ with a partially populated $\mathrm{LUMO} / \mathrm{d}_{\sigma}{ }^{*}$ do not ligate on the time scales probed here. This indicates that the excited-state reactivity of $\mathrm{Fe}(\mathrm{CO})_{4}$ can be correlated with orbital interactions and populations based on the capability of $2 p 3 d$ RIXS for probing the frontier orbitals. This study therefore demonstrates how, with time-resolved 2p3d RIXS, frontier-orbital interactions can be followed in time and locally at the probed metal site during the femtosecond electronic excited-state dynamics of metal complexes. This element-, site- and orbital-specific access to photo-chemical reaction mechanisms enables correlating orbital symmetry, spin multiplicity and reactivity of the complex and promises unique insight into the photochemical reaction dynamics of fundamentally important metal complexes, photocatalysts and bioinorganic systems. We note that the peculiarity of RIXS from electronic excited states with intensities arising at negative energy transfers where the outgoing photon energy is larger than the incident energy (anti-Stokes RIXS as observed experimentally and calculated for the excited-state singlet state of $\mathrm{Fe}(\mathrm{CO})_{4}$, Figure 20c) is detailed in ref. (Kunnus et al., 2016a).

\section{Conclusions and Outlook}

This chapter presents the basic principles of resonant inelastic x-ray scattering (RIXS) as the $\mathrm{x}$-ray analog of resonant Raman scattering for applications to $3 \mathrm{~d}$ transition-metal complexes, bio-inorganic metal systems and metal-based nanoparticles. RIXS in the soft and hard x-ray regimes probes the metal $L$ and $K$ absorption edges, respectively, and gives a metal-centric view of the electronic structure. With recent applications it is illustrated and discussed how RIXS gives information on ligand-field excitations, metal-ligand covalency, and electron or charge transfer in various systems ranging from simple metal complexes to metalloenzymes and nanoparticles. Time-resolved RIXS is presented as an emerging tool for characterizing transient short-lived reaction intermediates in photochemical reactions. Various challenges defining the frontiers of future applications of RIXS in chemistry can be discussed and the 
following choice is purely subjective and based on our own recent experience. Obviously, higher spectral and temporal resolutions always represent frontiers. An increase in spectral resolution with a bandwidth approaching $10 \mathrm{meV}$, or maybe even below, will further enhance insight into the ligand-field excitations and this may give additional access to symmetry distortions in metal-ligand systems. X-ray pulse durations on the order of $1 \mathrm{fs}$ at highly brilliant XFELs will enable non-linear versions of RIXS such as stimulated Raman scattering and this could be used to transfer the known non-linear spectroscopic techniques from the infrared and visible to the $x$-ray regime. Foremost, however, it seems of primary importance to further help making accessible in future RIXS experiments in chemistry socalled "relevant samples" to enable in particular systematic studies of catalysts, photocatalytic systems and metalloenzymes under in operando conditions. Making accessible such particularly dilute species in solution with concentrations below $1 \mathrm{mM}$ requires a number of improvements including advanced sample preparation schemes, highly brilliant $\mathrm{x}$-ray sources and, last but not least, dedicated $\mathrm{x}$-ray spectrometers. The recently commissioned and upcoming high-repetition rate XFELs, namely the EU-XFEL in Hamburg (Germany) and the LCLS-II in Stanford (USA) with, compared to current XFELs, increased average flux due to higher repetition rates will offer unique capabilities for time-resolved RIXS of such "relevant samples". Finally we emphasize that, as for every spectroscopic technique, theory is generally required to understand the information content of RIXS. The development of $a b$ initio quantum chemical methods including an explicit treatment of charge and spin densities in the ground and excited states of the system seems particularly promising for the description of metal-ligand bonds in these "relevant samples".

\section{References}

AL SAMARAI, M., DELGADO-JAIME, M. U., ISHII, H., HIRAOKA, N., TSUEI, K.-D., RUEFF, J. P., LASSALEKAISER, B., WECKHUYSEN, B. M. \& DE GROOT, F. M. 2016. 1s3p Resonant Inelastic X-ray Scattering of Cobalt Oxides and Sulfides. The Journal of Physical Chemistry C, 120, 2406324069.

AMENT, L. J. P., VAN VeENENDAAL, M., DEVEREAUX, T. P., HILL, J. P. \& VAN DEN BRINK, J. 2011. Resonant inelastic x-ray scattering studies of elementary excitations. Reviews of Modern Physics, 83, 705-767.

ASAKURA, D., NANBA, Y., OKUBO, M., MIZUNO, Y., NIWA, H., OSHIMA, M., ZHOU, H., OKADA, K. \& HARADA, Y. 2014. Distinguishing between High- and Low-Spin States for Divalent Mn in Mn- 
Based Prussian Blue Analogue by High-Resolution Soft X-ray Emission Spectroscopy. J Phys Chem Lett, 5, 4008-13.

BAKER, M. L., MARA, M. W., YAN, J. J., HODGSON, K. O., HEDMAN, B. \& SOLOMON, E. I. 2017. K- and L-edge X-ray Absorption Spectroscopy (XAS) and Resonant Inelastic X-ray Scattering (RIXS) Determination of Differential Orbital Covalency (DOC) of Transition Metal Sites. Coord Chem Rev, 345, 182-208.

BENCIVENGA, F., CUCINI, R., CAPOTONDI, F., BATTISTONI, A., MINCIGRUCCI, R., GIANGRISOSTOMI, E., GESSINI, A., MANFREdDA, M., NIKOLOV, I. P., PEDERSOLI, E., PRINCIPI, E., SVETINA, C., PARISSE, P., CASOLARI, F., DANAILOV, M. B., KISKINOVA, M. \& MASCIOVECCHIO, C. 2015. Four-wave mixing experiments with extreme ultraviolet transient gratings. Nature, 520, 205208.

BERGMANN, U. \& GLATZEL, P. 2009. X-ray emission spectroscopy. Photosynth Res, 102, 255-66.

BEYE, M., SCHRECK, S., SORGENFREI, F., TRABANT, C., PONTIUS, N., SCHUSSLER-LANGEHEINE, C., WURTH, W. \& FOHLISCH, A. 2013. Stimulated X-ray emission for materials science. Nature, 501, 191-4.

BOKAREV, S. I., KHAN, M., ABDEL-LATIF, M. K., XIAO, J., HILAL, R., AZIZ, S. G., AZIZ, E. F. \& KÜHN, O. 2015. Unraveling the Electronic Structure of Photocatalytic Manganese Complexes by L-Edge X-ray Spectroscopy. The Journal of Physical Chemistry C, 119, 19192-19200.

BRESSLER, C. \& CHERGUI, M. 2004. Ultrafast X-ray Absorption Spectroscopy. Chem Rev, 104, 17811812.

BUTORIN, S. M. 2000. Resonant inelastic X-ray scattering as a probe of optical scale excitations in strongly electron-correlated systems: quasi-localized view. Journal of Electron Spectroscopy and Related Phenomena, 110-111, 213-233.

BUTORIN, S. M., GUO, J., MAGNUSON, M., KUIPER, P. \& BNORDGREN, J. 1996a. Low-energy d-d excitations in $\mathrm{MnO}$ studied by resonant $\mathrm{x}$-ray fluorescence spectroscopy. Phys. Rev. B, 54, 4405-4408.

BUTORIN, S. M., GUO, J. H., MAGNUSON, M., KUIPER, P. \& NORDGREN, J. 1996b. Low-energy d-d excitations in $\mathrm{MnO}$ studied by resonant x-ray fluorescence spectroscopy. Physical Review B, 54, 4405-4408.

BUTORIN, S. M., MANCINI, D. C., GUO, J. H., WASSDAHL, N., NORDGREN, J., NAKAZAWA, M., TANAKA, S., UOZUMI, T., KOTANI, A., MA, Y., MYANO, K. E., KARLIN, B. A. \& SHUH, D. K. 1996c. Resonant X-ray fluorescence spectroscopy of correlated systems: A probe of charge-transfer excitations. Physical Review Letters, 77, 574-577.

CANTON, S. E., KJAER, K. S., VANKO, G., VAN DRIEL, T. B., ADACHI, S., BORDAGE, A., BRESSLER, C., CHABERA, P., CHRISTENSEN, M., DOHN, A. O., GALLER, A., GAWELDA, W., GOSZTOLA, D., HALDRUP, K., HARLANG, T., LIU, Y., MOLLER, K. B., NEMETH, Z., NOZAWA, S., PAPAI, M., SATO, T., SATO, T., SUAREZ-ALCANTARA, K., TOGASHI, T., TONO, K., UHLIG, J., VITHANAGE, D. A., WARNMARK, K., YABASHI, M., ZHANG, J., SUNDSTROM, V. \& NIELSEN, M. M. 2015. Visualizing the non-equilibrium dynamics of photoinduced intramolecular electron transfer with femtosecond X-ray pulses. Nat Commun, 6, 6359.

CHEN, L. X. 2005. Probing transient molecular structures in photochemical processes using laserinitiated time-resolved X-ray absorption spectroscopy. Annu Rev Phys Chem, 56, 221-54.

CHERGUI, M. \& COLLET, E. 2017. Photoinduced Structural Dynamics of Molecular Systems Mapped by Time-Resolved X-ray Methods. Chem Rev, 117, 11025-11065.

CORDONES, A. A., LEE, J. H., HONG, K., CHO, H., GARG, K., BOGGIO-PASQUA, M., RACK, J. J., HUSE, N., SCHOENLEIN, R. W. \& KIM, T. K. 2018. Transient metal-centered states mediate isomerization of a photochromic ruthenium-sulfoxide complex. Nat Commun, 9, 1989.

CUI, Z., XIE, C., FENG, X., BECKNELL, N., YANG, P., LU, Y., ZHAI, X., LIU, X., YANG, W., CHUANG, Y. D. \& GUO, J. 2017. Revealing the Size-Dependent d-d Excitations of Cobalt Nanoparticles Using Soft X-ray Spectroscopy. J Phys Chem Lett, 8, 319-325.

DE GROOT, F. 2001. High-Resolution X-ray Emission and X-ray Absorption Spectroscopy. Chem Rev, $101,1779-1808$. 
DE GROOT, F. \& KOTANI, A. 2008. Core Level Spectroscopy of Solids, CRC Press Taylor and Francis Group.

DELL'ANGELA, M., ANNIYEV, T., BEYE, M., COFFEE, R., FOHLISCH, A., GLADH, J., KATAYAMA, T., KAYA, S., KRUPIN, O., LARUE, J., MOGELHOJ, A., NORDLUND, D., NORSKOV, J. K., OBERG, H., OGASAWARA, H., OSTROM, H., PETTERSSON, L. G., SCHLOTTER, W. F., SELlBERG, J. A., SORGENFREI, F., TURNER, J. J., WOLF, M., WURTH, W. \& NILSSON, A. 2013. Real-time observation of surface bond breaking with an x-ray laser. Science, 339, 1302-5.

ECKERT, S., NORELL, J., MIEDEMA, P. S., BEYE, M., FONDELL, M., QUEVEDO, W., KENNEDY, B., HANTSCHMANN, M., PIETZSCH, A., VAN KUIKEN, B. E., ROSS, M., MINITTI, M. P., MOELLER, S. P., SCHLOTTER, W. F., KHALIL, M., ODELIUS, M. \& FOHLISCH, A. 2017. Ultrafast Independent $\mathrm{N}-\mathrm{H}$ and N-C Bond Deformation Investigated with Resonant Inelastic X-Ray Scattering. Angew Chem Int Ed Engl, 56, 6088-6092.

ENGEL, N., BOKAREV, S. I., SULJOTI, E., GARCIA-DIEZ, R., LANGE, K. M., ATAK, K., GOLNAK, R., KOTHE, A., DANTZ, M. \& KÜHN, O. 2014. Chemical bonding in aqueous ferrocyanide: experimental and theoretical X-ray spectroscopic study. The Journal of Physical Chemistry B, 118, 15551563.

FIGGIS, B. N. 1966. Introduction to Ligand Fields, Interscience Publishers, John Wiley \& Sons, Inc.

FORTE, F., AMENT, L. J. \& VAN DEN BRINK, J. 2008. Single and double orbital excitations probed by resonant inelastic x-ray scattering. Physical review letters, 101, 106406-(1-4).

GALLO, E., GORELOV, E., GUDA, A. A., BUGAEV, A. L., BONINO, F., BORFECCHIA, E., RICCHIARDI, G., GIANOLIO, D., CHAVAN, S. \& LAMBERTI, C. 2017. Effect of Molecular Guest Binding on the dd Transitions of Ni2+ of CPO-27-Ni: A Combined UV-Vis, Resonant-Valence-to-Core X-ray Emission Spectroscopy, and Theoretical Study. Inorganic chemistry, 56, 14408-14425.

GEL'MUKHANOV, F. \& ÅGREN, H. 1999. Resonant X-ray Raman scattering. Physics Reports, 312, 87330.

GLATZEL, P. \& BERGMANN, U. 2005. High resolution 1s core hole X-ray spectroscopy in 3d transition metal complexes - electronic and structural information. Coordination Chemistry Reviews, 249, 65-95.

GLATZEL, P., BERGMANN, U., YANO, J., VISSER, H., ROBBLEE, J. H., GU, W. W., DE GROOT, F. M. F., CHRISTOU, G., PECORARO, V. L., CRAMER, S. P. \& YACHANDRA, V. K. 2004. The electronic structure of $\mathrm{Mn}$ in oxides, coordination complexes, and the oxygen-evolving complex of photosystem II studied by resonant inelastic X-ray scattering. Journal of the American Chemical Society, 126, 9946-9959.

GLATZEL, P., SCHROEDER, H., PUSHKAR, Y., BORON III, T., MUKHERJEE, S., CHRISTOU, G., PECORARO, V. L., MESSINGER, J., YACHANDRA, V. K. \& BERGMANN, U. 2013. Electronic structural changes of $\mathrm{Mn}$ in the oxygen-evolving complex of photosystem II during the catalytic cycle. Inorganic chemistry, 52, 5642-5644.

GOTZ, M. D., SOLDATOV, M. A., LANGE, K. M., ENGEL, N., GOLNAK, R., KÖNNECKE, R., ATAK, K., EBERHARDT, W. \& AZIZ, E. F. 2012. Probing Coster-Kronig Transitions in Aqueous Fe2+ Solution Using Inverse Partial and Partial Fluorescence Yield at the L-Edge. The Journal of Physical Chemistry Letters, 3, 1619-1623.

GUO, J.-H., LUO, Y., AUGUSTSSON, A., RUBENSSON, J.-E., SÅTHE, C., ÅGREN, H., SIEGBAHN, H. \& NORDGREN, J. 2002. X-ray emission spectroscopy of hydrogen bonding and electronic structure of liquid water. Physical Review Letters, 89, 137402.

GUO, M., KÄLLMAN, E., SØRENSEN, L. K., DELCEY, M. G., PINJARI, R. V. \& LUNDBERG, M. 2016. Molecular Orbital Simulations of Metal 1s2p Resonant Inelastic X-ray Scattering. The Journal of Physical Chemistry A, 120, 5848-5855.

HADT, R. G., HAYES, D., BRODSKY, C. N., ULLMAN, A. M., CASA, D. M., UPTON, M. H., NOCERA, D. G. \& CHEN, L. X. 2016. X-ray Spectroscopic Characterization of Co (IV) and Metal-Metal Interactions in Co4O4: Electronic Structure Contributions to the Formation of High-Valent States Relevant to the Oxygen Evolution Reaction. Journal of the American Chemical Society, 138, 11017-11030. 
HAHN, A. W., VAN KUIKEN, B. E., AL SAMARAI, M., ATANASOV, M., WEYHERMULLER, T., CUI, Y. T., MIYAWAKI, J., HARADA, Y., NICOLAOU, A. \& DEBEER, S. 2017. Measurement of the Ligand Field Spectra of Ferrous and Ferric Iron Chlorides Using 2p3d RIXS. Inorg Chem, 56, 82038211.

HARADA, Y., TAKEUCHI, T., KINO, H., FUKUSHIMA, A., TAKAKURA, K., HIEDA, K., NAKAO, A., SHIN, S. \& FUKUYAMA, H. 2006. Electronic Structure of DNA Nucleobases and Their Dinucleotides Explored by Soft X-ray Spectroscopy. J. Phys. Chem. A, 110, 13227-13231.

HOCKING, R. K., WASINGER, E. C., YAN, Y. L., DEGROOT, F. M. F., WALKER, F. A., HODGSON, K. O., HEDMAN, B. \& SOLOMON, E. I. 2007. Fe L-edge x-ray absorption spectroscopy of low-spin heme relative to non-heme Fe complexes: Delocalization of Fe d-electrons into the porphyrin ligand. Journal of the American Chemical Society, 129, 113-125.

HONG, K., CHO, H., SCHOENLEIN, R. W., KIM, T. K. \& HUSE, N. 2015. Element-specific characterization of transient electronic structure of solvated $\mathrm{Fe}(\mathrm{II})$ complexes with time-resolved soft X-ray absorption spectroscopy. Acc Chem Res, 48, 2957-66.

HUNAULT, M., HARADA, Y., MIYAWAKI, J., WANG, J., MEIJERINK, A., DE GROOT, F. M. F. \& VAN SCHOONEVELD, M. M. 2018. Direct Observation of $\mathrm{Cr}(3+)$ 3d States in Ruby: Toward Experimental Mechanistic Evidence of Metal Chemistry. J Phys Chem A, 122, 4399-4413.

JAY, R. M., NORELL, J., ECKERT, S., HANTSCHMANN, M., BEYE, M., KENNEDY, B., QUEVEDO, W., SCHLOTTER, W. F., DAKOVSKI, G. L. \& MINITTI, M. P. 2018a. Disentangling Transient Charge Density and Metal-Ligand Covalency in Photo-Excited Ferricyanide with Femtosecond Resonant Inelastic Soft X-ray Scattering. The journal of physical chemistry letters.

JAY, R. M., NORELL, J., ECKERT, S., HANTSCHMANN, M., BEYE, M., KENNEDY, B., QUEVEDO, W., SCHLOTTER, W. F., DAKOVSKI, G. L., MINITTI, M. P., HOFFMANN, M. C., MITRA, A., MOELLER, S. P., NORDLUND, D., ZHANG, W., LIANG, H. W., KUNNUS, K., KUBICEK, K., TECHERT, S. A., LUNDBERG, M., WERNET, P., GAFFNEY, K., ODELIUS, M. \& FOHLISCH, A. 2018b. Disentangling Transient Charge Density and Metal-Ligand Covalency in Photoexcited Ferricyanide with Femtosecond Resonant Inelastic Soft X-ray Scattering. J Phys Chem Lett, 9, 3538-3543.

JOSEFSSON, I., KUNNUS, K., SCHRECK, S., FOHLISCH, A., DE GROOT, F., WERNET, P. \& ODELIUS, M. 2012. Ab Initio Calculations of X-ray Spectra: Atomic Multiplet and Molecular Orbital Effects in a Multiconfigurational SCF Approach to the L-Edge Spectra of Transition Metal Complexes. J Phys Chem Lett, 3, 3565-70.

KAO, C.-C., CALIEBE, W., HASTINGS, J. \& GILLET, J.-M. 1996. X-ray resonant Raman scattering in NiO: Resonant enhancement of the charge-transfer excitations. Physical Review B, 54, 1636116364.

KIM, K. H., KIM, J., OANG, K. Y., LEE, J. H., GROLIMUND, D., MILNE, C. J., PENFOLD, T. J., JOHNSON, S. L., GALLER, A., KIM, T. W., KIM, J. G., SUH, D., MOON, J., KIM, J., HONG, K., GUERIN, L., KIM, T. K., WULFF, M., BRESSLER, C. \& IHEE, H. 2015a. Identifying the major intermediate species by combining time-resolved $\mathrm{X}$-ray solution scattering and $\mathrm{X}$-ray absorption spectroscopy. Phys Chem Chem Phys, 17, 23298-302.

KIM, K. H., KIM, J. G., NOZAWA, S., SATO, T., OANG, K. Y., KIM, T. W., KI, H., JO, J., PARK, S., SONG, C., SATO, T., OGAWA, K., TOGASHI, T., TONO, K., YABASHI, M., ISHIKAWA, T., KIM, J., RYOO, R., $\mathrm{KIM}$, J., IHEE, H. \& ADACHI, S. 2015b. Direct observation of bond formation in solution with femtosecond X-ray scattering. Nature, 518, 385-9.

KOTANI, A. \& SHIN, S. 2001. Resonant inelastic x-ray scattering spectra for electrons in solids. Reviews of Modern Physics, 73, 203-246.

KOZIEJ, D. \& DEBEER, S. 2017. Application of Modern X-ray Spectroscopy in Chemistry-Beyond Studying the Oxidation State. Chemistry of Materials, 29, 7051-7053.

KROLL, T., HADT, R. G., WILSON, S. A., LUNDBERG, M., YAN, J. J., WENG, T.-C., SOKARAS, D., ALONSOMORI, R., CASA, D., UPTON, M. H., HEDMAN, B., HODGSON, K. O. \& SOLOMON, E. I. 2014. Resonant Inelastic X-ray Scattering on Ferrous and Ferric bis-imidazole Porphyrin and Cytochrome c: Nature and Role of the Axial Methionine-Fe Bond. Journal of the American Chemical Society. 
KROLL, T., WENINGER, C., ALONSO-MORI, R., SOKARAS, D., ZHU, D., MERCADIER, L., MAJETY, V. P., MARINELLI, A., LUTMAN, A., GUETG, M. W., DECKER, F. J., BOUTET, S., AQUILA, A., KOGLIN, J., KORALEK, J., DEPONTE, D. P., KERN, J., FULLER, F. D., PASTOR, E., FRANSSON, T., ZHANG, Y., YANO, J., YACHANDRA, V. K., ROHRINGER, N. \& BERGMANN, U. 2018. Stimulated X-Ray Emission Spectroscopy in Transition Metal Complexes. Phys Rev Lett, 120, 133203.

KUBIN, M., KERN, J., GUL, S., KROLL, T., CHATTERJEe, R., LOCHEL, H., FULLER, F. D., SIERRA, R. G., QUEVEDO, W., WENIGER, C., REHANEK, J., FIRSOV, A., LAKSMONO, H., WENINGER, C., ALONSO-MORI, R., NORDLUND, D. L., LASSALLE-KAISER, B., GLOWNIA, J. M., KRZYWINSKI, J., MOELLER, S., TURNER, J. J., MINITTI, M. P., DAKOVSKI, G. L., KOROIDOV, S., KAWDE, A., KANADY, J. S., TSUI, E. Y., SUSENO, S., HAN, Z., HILL, E., TAGUCHI, T., BOROVIK, A. S., AGAPIE, T., MESSINGER, J., ERKO, A., FOHLISCH, A., BERGMANN, U., MITZNER, R., YACHANDRA, V. K., YANO, J. \& WERNET, P. 2017. Soft $x$-ray absorption spectroscopy of metalloproteins and high-valent metal-complexes at room temperature using free-electron lasers. Struct Dyn, 4, 054307.

KUIPER, P., GUO, J., SATHE, C., DUDA, L.-C., NORDGREN, J., POTHUIZEN, J. J. M., DE GROOT, F. M. \& SAWATZKY, G. A. 1998. Resonant X-Ray Raman Spectra of Cu dd Excitations in Sr2CuO2Cl2. Phys. Rev. Lett., 1998, 5204-5207.

KUNNUS, K., JOSEFSSON, I., RAJKOVIC, I., SCHRECK, S., QUEVEDO, W., BEYE, M., GRÜBEL, S., SCHOLZ, M., NORDLUND, D., ZHANG, W., HARTSOCK, R. W., GAFFNEY, K. J., SCHLOTTER, W. F., TURNER, J. J., KENNEDY, B., HENNIES, F., TECHERT, S., WERNET, P., ODELIUS, M. \& FÖHLISCH, A. 2016a. Anti-Stokes resonant $x$-ray Raman scattering for atom specific and excited state selective dynamics. New Journal of Physics, 18, 103011.

KUNNUS, K., JOSEFSSON, I., RAJKOVIC, I., SCHRECK, S., QUEVEDO, W., BEYE, M., WENIGER, C., GRUBEL, S., SCHOLZ, M., NORDLUND, D., ZHANG, W., HARTSOCK, R. W., GAFFNEY, K. J., SCHLOTTER, W. F., TURNER, J. J., KENNEDY, B., HENNIES, F., DE GROOT, F. M., TECHERT, S., ODELIUS, M., WERNET, P. \& FOHLISCH, A. 2016b. Identification of the dominant photochemical pathways and mechanistic insights to the ultrafast ligand exchange of $\mathrm{Fe}(\mathrm{CO}) 5$ to $\mathrm{Fe}(\mathrm{CO}) 4 \mathrm{EtOH}$. Struct Dyn, 3, 043204.

KUNNUS, K., JOSEFSSON, I., SCHRECK, S., QUEVEDO, W., MIEDEMA, P. S., TECHERT, S., DE GROOT, F. M., ODELIUS, M., WERNET, P. \& FOHLISCH, A. 2013. From ligand fields to molecular orbitals: probing the local valence electronic structure of $\mathrm{Ni}(2+)$ in aqueous solution with resonant inelastic X-ray scattering. J Phys Chem B, 117, 16512-21.

KUNNUS, K., JOSEFSSON, I., SCHRECK, S., QUEVEDO, W., MIEDEMA, P. S., TECHERT, S., DE GROOT, F. M. F., FÖHLISCH, A., ODELIUS, M. \& WERNET, P. 2017. Quantifying covalent interactions with resonant inelastic soft X-ray scattering: Case study of Ni2+ aqua complex. Chemical Physics Letters, 669, 196-201.

KUNNUS, K., RAJKOVIC, I., SCHRECK, S., QUEVEDO, W., ECKERT, S., BEYE, M., SULJOTI, E., WENIGER, C., KALUS, C. \& GRÜBEL, S. 2012. A setup for resonant inelastic soft $x$-ray scattering on liquids at free electron laser light sources. Review of Scientific Instruments, 83, 123109.

KUNNUS, K., ZHANG, W., DELCEY, M. G., PINJARI, R. V., MIEDEMA, P. S., SCHRECK, S., QUEVEDO, W., SCHRÖDER, H., FÖHLISCH, A., GAFFNEY, K. J., LUNDBERG, M., ODELIUS, M. \& WERNET, P. 2016c. Viewing the Valence Electronic Structure of Ferric and Ferrous Hexacyanide in Solution from the Fe and Cyanide Perspectives. The Journal of Physical Chemistry B, 120, 7182-7194.

LANGE, K. M., KÖNNECKE, R., GHADIMI, S., GOLNAK, R., SOLDATOV, M. A., HODECK, K. F., SOLDATOV, A. \& AZIZ, E. F. 2010. High resolution X-ray emission spectroscopy of water and aqueous ions using the micro-jet technique. Chemical Physics, 377, 1-5.

LEIDEL, N., CHERNEV, P., HAVELIUS, K. G., SCHWARTZ, L., OTT, S. \& HAUMANN, M. 2012. Electronic Structure of an [FeFe] Hydrogenase Model Complex in Solution Revealed by X-ray Absorption Spectroscopy Using Narrow-Band Emission Detection. Journal of the American Chemical Society, 134, 14142-14157. 
LEVANTINO, M., SCHIRO, G., LEMKE, H. T., COTTONE, G., GLOWNIA, J. M., ZHU, D., CHOLLeT, M., IHEE, H., CUPANE, A. \& CAMMARATA, M. 2015. Ultrafast myoglobin structural dynamics observed with an X-ray free-electron laser. Nat Commun, 6, 6772.

LIU, B., VAN SCHOONEVELD, M. M., CUI, Y. T., MIYAWAKI, J., HARADA, Y., ESCHEMANN, T. O., DE JONG, K. P., DELGADO-JAIME, M. U. \& DE GROOT, F. M. F. 2017. In-Situ 2p3d Resonant Inelastic X-ray Scattering Tracking Cobalt Nanoparticle Reduction. J Phys Chem C Nanomater Interfaces, 121, 17450-17456.

LIU, B., WANG, R. P., GLASS, E. N., HILL, C. L., CUK, T., OKAMOTO, J., HUANG, D. J., VAN SCHOONEVELD, M. M. \& DE GROOT, F. M. 2016. Distorted Tetrahedral Co(II) in $\mathrm{K} 5 \mathrm{H}[\mathrm{CoW} 12 \mathrm{O} 40] . \mathrm{xH} 2 \mathrm{O}$ Probed by 2p3d Resonant Inelastic X-ray Scattering. Inorg Chem, 55, $10152-10160$.

LUNDBERG, M., KROLL, T., DEBEER, S., BERGMANN, U., WILSON, S. A., GLATZEL, P., NORDLUND, D., HEDMAN, B., HODGSON, K. O. \& SOLOMON, E. I. 2013. Metal-Ligand Covalency of Iron Complexes from High-Resolution Resonant Inelastic X-ray Scattering. Journal of the American Chemical Society, 135, 17121-17134.

MACMILLAN, S. N. \& LANCASTER, K. M. 2017. X-ray Spectroscopic Interrogation of Transition-MetalMediated Homogeneous Catalysis: Primer and Case Studies. ACS Catalysis, 7, 1776-1791.

MARA, M. W., HADT, R. G., REINHARD, M., KROLL, T., LIM, H., HARTSOCK, R. W., ALONSO-MORI, R., CHOLLET, M., GLOWNIA, J. M., NELSON, S., SOKARAS, D., KUNNUS, K., HODGSON, K. O., HEDMAN, B., BERGMANN, U., GAFFNEY, K. \& SOLOMON, E. I. 2017. Metalloprotein entatic control of ligand-metal bonds quantified by ultrafast $x$-ray spectroscopy. Science, 356, 12761280.

MARCH, A. M., ASSEFA, T. A., BRESSLER, C., DOUMY, G., GALLER, A., GAWELDA, W., KANTER, E. P., NEMETH, Z., PAPAI, M., SOUTHWORTH, S. H., YOUNG, L. \& VANKO, G. 2015. Feasibility of Valence-to-Core X-ray Emission Spectroscopy for Tracking Transient Species. J Phys Chem $C$ Nanomater Interfaces, 119, 14571-14578.

MARCHENKO, T., CARNIATO, S., JOURNEL, L., GUILLEMIN, R., KAWERK, E., ŽITNIK, M., KAVČIČ, M., BUČAR, K., BOHINC, R., PETRIC, M., VAZ DA CRUZ, V., GEL'MUKHANOV, F. \& SIMON, M. 2015. Electron Dynamics in the Core-ExcitedCS2Molecule Revealed through Resonant Inelastic XRay Scattering Spectroscopy. Physical Review $X, 5$.

MEYER, D. A., ZHANG, X. N., BERGMANN, U. \& GAFFNEY, K. J. 2010. Characterization of charge transfer excitations in hexacyanomanganate(III) with $\mathrm{Mn}$ K-edge resonant inelastic x-ray scattering. Journal of Chemical Physics, 132, 134502-(1-7).

MIEDEMA, P. S., MITZNER, R., GANSCHOW, S., FOHLISCH, A. \& BEYE, M. 2017. X-ray spectroscopy on the active ion in laser crystals. Phys Chem Chem Phys, 19, 21800-21806.

MILLER, N. A., DEB, A., ALONSO-MORI, R., GARABATO, B. D., GLOWNIA, J. M., KIEFER, L. M., KORALEK, J., SIKORSKI, M., SPEARS, K. G., WILEY, T. E., ZHU, D., KOZLOWSKI, P. M., KUBARYCH, K. J., PENNER-HAHN, J. E. \& SENSION, R. J. 2017. Polarized XANES Monitors Femtosecond Structural Evolution of Photoexcited Vitamin B12. J Am Chem Soc, 139, 1894-1899.

MILNE, C. J., PENFOLD, T. J. \& CHERGUI, M. 2014. Recent experimental and theoretical developments in time-resolved X-ray spectroscopies. Coordination Chemistry Reviews, 277-278, 44-68.

NILSSON, A. \& PETTERSSON, L. G. M. 2004. Chemical bonding on surfaces probed by X-ray emission spectroscopy and density functional theory. Surface Science Reports, 55, 49-167.

ÖSTRÖM, H., ÖBERG, H., XIN, H., LARUE, J., BEYE, M., DELL'ANGELA, M., GLADH, J., NG, M. L., SELLBERG, J. A., KAYA, S., MERCURIO, G., NORDLUND, D., HANTSCHMANN, M., HIEKE, F., KÜHN, D., SCHLOTTER, W. F., DAKOVSKI, G. L., TURNER, J. J., MINITTI, M. P., MITRA, A., MOELLER, S., FÖHLISCH, A., WOLF, M., WURTH, W., PERSSON, P., NORSKOV, J. K., ABILDPEDERSEN, F., OGASAWARA, H., PETTERSSON, L. G. \& NILSSON, A. 2015. Probing the transition state region in catalytic $\mathrm{CO}$ oxidation on Ru. Science, 347, 978-982.

PENFOLD, T. J., REINHARD, M., RITTMANN-FRANK, M. H., TAVERNELLI, I., ROTHLISBERGER, U., MILNE, C. J., GLATZEL, P. \& CHERGUI, M. 2014. X-ray spectroscopic study of solvent effects on the ferrous and ferric hexacyanide anions. The Journal of Physical Chemistry A, 118, 9411-9418. 
PENFOLD, T. J., SZLACHETKO, J., SANTOMAURO, F. G., BRITZ, A., GAWELDA, W., DOUMY, G., MARCH, A. M., SOUTHWORTH, S. H., RITTMANN, J., ABELA, R., CHERGUI, M. \& MILNE, C. J. 2018. Revealing hole trapping in zinc oxide nanoparticles by time-resolved $\mathrm{X}$-ray spectroscopy. Nat Commun, 9, 478.

PINJARI, R. V., DELCEY, M. G., GUO, M., ODELIUS, M. \& LUNDBERG, M. 2014. Restricted active space calculations of L-edge X-ray absorption spectra: from molecular orbitals to multiplet states. J Chem Phys, 141, 124116.

PLATZMAN, P. M. \& ISAACS, E. D. 1998. Resonant inelastic x-ray scattering. Physical Review B, 57, 11107-11114.

POLLOCK, C. J. \& DEBEER, S. 2015. Insights into the geometric and electronic structure of transition metal centers from valence-to-core X-ray emission spectroscopy. Acc Chem Res, 48, 2967-75.

PREUSSE, M., BOKAREV, S. I., AZIZ, S. G. \& KUHN, O. 2016. Towards an ab initio theory for metal Ledge soft X-ray spectroscopy of molecular aggregates. Struct Dyn, 3, 062601.

ROEMELT, M., MAGANAS, D., DEBEER, S. \& NEESE, F. 2013. A combined DFT and restricted open-shell configuration interaction method including spin-orbit coupling: application to transition metal L-edge X-ray absorption spectroscopy. J Chem Phys, 138, 204101.

ROHRINGER, N., RYAN, D., LONDON, R. A., PURVIS, M., ALBERT, F., DUNN, J., BOZEK, J. D., BOSTEDT, C., GRAF, A., HILL, R., HAU-RIEGE, S. P. \& ROCCA, J. J. 2012. Atomic inner-shell X-ray laser at 1.46 nanometres pumped by an X-ray free-electron laser. Nature, 481, 488-91.

SULJOTI, E., GARCIA-DIEZ, R., BOKAREV, S. I., LANGE, K. M., SCHOCH, R., DIERKER, B., DANTZ, M., YAMAMOTO, K., ENGEL, N., ATAK, K., KUHN, O., BAUER, M., RUBENSSON, J. E. \& AZIZ, E. F. 2013. Direct observation of molecular orbital mixing in a solvated organometallic complex. Angew Chem Int Ed Engl, 52, 9841-4.

VAN DER LAAN, G. \& KIRKMAN, I. W. 1992. The $2 p$ absorption spectra of 3d transition metal compounds in tetrahedral and octahedral symmetry. J. Phys.: Condens. Matter, 4, 41894204.

VAN KUIKEN, B. E., HAHN, A. W., MAGANAS, D. \& DEBEER, S. 2016. Measuring Spin-Allowed and SpinForbidden $d$-d Excitations in Vanadium Complexes with 2p3d Resonant Inelastic X-ray Scattering. Inorg Chem, 55, 11497-11501.

VAN KUIKEN, B. E., HAHN, A. W., NAYYAR, B., SCHIEWER, C. E., LEE, S. C., MEYER, F., WEYHERMULLER, T., NICOLAOU, A., CUI, Y. T., MIYAWAKI, J., HARADA, Y. \& DEBEER, S. 2018. Electronic Spectra of Iron-Sulfur Complexes Measured by 2p3d RIXS Spectroscopy. Inorg Chem, 57, 7355-7361.

VAN SCHOONEVELD, M. M., GOSSELINK, R. W., EGGENHUISEN, T. M., AL SAMARAI, M., MONNEY, C., ZHOU, K. J., SCHMITT, T. \& DE GROOT, F. M. 2013a. A multispectroscopic study of 3d orbitals in cobalt carboxylates: the high sensitivity of $2 \mathrm{p} 3 \mathrm{~d}$ resonant $\mathrm{X}$-ray emission spectroscopy to the ligand field. Angew Chem Int Ed Engl, 52, 1170-4.

VAN SCHOONEVELD, M. M., JUHIN, A., CAMPOS-CUERVA, C., SCHMITT, T. \& DE GROOT, F. M. F. 2013b. Origin of Low Energy $d-d$ Excitations Observed on Wet Chemically Prepared Cobalt Bearing Nanoparticles by 2p3d Resonant X-ray Emission Spectroscopy. The Journal of Physical Chemistry C, 117, 14398-14407.

VANKÓ, G., BORDAGE, A., GLATZEL, P., GALLO, E., ROVEZZI, M., GAWELDA, W., GALLER, A., BRESSLER, C., DOUMY, G., MARCH, A. M., KANTER, E. P., YOUNG, L., SOUTHWORTH, S. H., CANTON, S. E., UHLIG, J., SMOLENTSEV, G., SUNDSTRÖM, V., HALDRUP, K., VAN DRIEL, T. B., NIELSEN, M. M., KJAER, K. S. \& LEMKE, H. T. 2013. Spin-state studies with XES and RIXS: From static to ultrafast. Journal of Electron Spectroscopy and Related Phenomena, 188, 166-171.

VANKO, G., BORDAGE, A., PAPAI, M., HALDRUP, K., GLATZEL, P., MARCH, A. M., DOUMY, G., BRITZ, A., GALLER, A., ASSEFA, T., CABARET, D., JUHIN, A., VAN DRIEL, T. B., KJAER, K. S., DOHN, A., MOLLER, K. B., LEMKE, H. T., GALLO, E., ROVEZZI, M., NEMETH, Z., ROZSALYI, E., ROZGONYI, T., UHLIG, J., SUNDSTROM, V., NIELSEN, M. M., YOUNG, L., SOUTHWORTH, S. H., BRESSLER, C. \& GAWELDA, W. 2015. Detailed Characterization of a Nanosecond-Lived Excited State: Xray and Theoretical Investigation of the Quintet State in Photoexcited [Fe(terpy)2](2.). J Phys Chem C Nanomater Interfaces, 119, 5888-5902. 
VAZ DA CRUZ, V., ERTAN, E., COUTO, R. C., ECKERT, S., FONDELL, M., DANTZ, M., KENNEDY, B., SCHMITT, T., PIETZSCH, A., GUIMARAES, F. F., AGREN, H., GEL'MUKHANOV, F., ODELIUS, M., FOHLISCH, A. \& KIMBERG, V. 2017. A study of the water molecule using frequency control over nuclear dynamics in resonant X-ray scattering. Phys Chem Chem Phys, 19, 19573-19589.

WANG, R. P., LIU, B., GREEN, R. J., DELGADO-JAIME, M. U., GHIASI, M., SCHMITT, T., VAN SCHOONEVELD, M. M. \& DE GROOT, F. M. F. 2017. Charge-Transfer Analysis of 2p3d Resonant Inelastic X-ray Scattering of Cobalt Sulfide and Halides. J Phys Chem C Nanomater Interfaces, 121, 24919-24928.

WASINGER, E. C., DE GROOT, F., HEDMAN, B., HODGSON, K. O. \& SOLOMON, E. I. 2003. L-edge X-ray Absorption Spectroscopy of Non-Heme Iron Sites: Experimental Determination of Differential Orbital Covalency. J. Am. Chem. Soc., 125, 12894-12906.

WEINHARDT, L., ERTAN, E., IANNUZZI, M., WEIGAND, M., FUCHS, O., BAR, M., BLUM, M., DENLINGER, J. D., YANG, W., UMBACH, E., ODELIUS, M. \& HESKE, C. 2015. Probing hydrogen bonding orbitals: resonant inelastic soft X-ray scattering of aqueous NH3. Phys Chem Chem Phys, 17, 27145-53.

WERNET, P. 2017. Orbital-specific Mapping of Chemical Interactions and Dynamics with Femtosecond Soft X-ray Pulses. In: YANO, J., YACHANDRA, V. \& BERGMANN, U. (eds.) X-Ray Free Electron Lasers. Royal Society of Chemistry: Royal Society of Chemistry.

WERNET, P., KUNNUS, K., JOSEFSSON, I., RAJKOVIC, I., QUEVEDO, W., BEYE, M., SCHRECK, S., GRUBEL, S., SCHOLZ, M., NORDLUND, D., ZHANG, W., HARTSOCK, R. W., SCHLOTTER, W. F., TURNER, J. J., KENNEDY, B., HENNIES, F., DE GROOT, F. M., GAFFNEY, K. J., TECHERT, S., ODELIUS, M. \& FOHLISCH, A. 2015. Orbital-specific mapping of the ligand exchange dynamics of $\mathrm{Fe}(\mathrm{CO}) 5$ in solution. Nature, 520, 78-81.

WERNET, P., KUNNUS, K., SCHRECK, S., QUEVEDO, W., KURIAN, R., TECHERT, S., DE GROOT, F. M., ODELIUS, M. \& FOHLISCH, A. 2012. Dissecting Local Atomic and Intermolecular Interactions of Transition-Metal Ions in Solution with Selective X-ray Spectroscopy. J Phys Chem Lett, 3, 3448-53.

WILSON, S. A., KROLL, T., DECREAU, R. A., HOCKING, R. K., LUNDBERG, M., HEDMAN, B., HODGSON, K. O. \& SOLOMON, E. I. 2013. Iron L-edge X-ray absorption spectroscopy of oxy-picket fence porphyrin: Experimental insight into Fe-O2 bonding. Journal of the American Chemical Society, 135, 1124.

YONEDA, H., INUBUSHI, Y., NAGAMINE, K., MICHINE, Y., OHASHI, H., YUMOTO, H., YAMAUCHI, K., MIMURA, H., KITAMURA, H., KATAYAMA, T., ISHIKAWA, T. \& YABASHI, M. 2015. Atomic inner-shell laser at 1.5-angstrom wavelength pumped by an X-ray free-electron laser. Nature, 524, 446-9.

ZHANG, W., ALONSO-MORI, R., BERGMANN, U., BRESSLER, C., CHOLLET, M., GALLER, A., GAWELDA, W., HADT, R. G., HARTSOCK, R. W., KROLL, T., KJAER, K. S., KUBICEK, K., LEMKE, H. T., LIANG, H. W., MEYER, D. A., NIELSEN, M. M., PURSER, C., ROBINSON, J. S., SOLOMON, E. I., SUN, Z., SOKARAS, D., VAN DRIEL, T. B., VANKO, G., WENG, T. C., ZHU, D. \& GAFFNEY, K. J. 2014. Tracking excited-state charge and spin dynamics in iron coordination complexes. Nature, 509, 345-8.

ZHANG, W. \& GAFFNEY, K. J. 2015. Mechanistic studies of photoinduced spin crossover and electron transfer in inorganic complexes. Acc Chem Res, 48, 1140-8.

ZITNIK, M., KAVCIC, M., BOHINC, R., BUCAR, K., MIHELIC, A., CAO, W., GUILLEMIN, R., JOURNEL, L., MARCHENKO, T., CARNIATO, S., KAWERK, E., PIANCASTELLI, M. N. \& SIMON, M. 2015. Resonant inelastic X-ray spectroscopy of atoms and simple molecules: Satellite features and dependence on energy detuning and photon polarization. Journal of Electron Spectroscopy and Related Phenomena, 204, 356-364. 